\title{
ON THE GEOMETRY OF GOURSAT STRUCTURES
}

\author{
William PAsillas-LÉPine ${ }^{1}$ AND Witold ReSPONDEK ${ }^{2}$
}

\begin{abstract}
A Goursat structure on a manifold of dimension $n$ is a rank two distribution $\mathcal{D}$ such that $\operatorname{dim} \mathcal{D}^{(i)}=i+2$, for $0 \leq i \leq n-2$, where $\mathcal{D}^{(i)}$ denote the elements of the derived flag of $\mathcal{D}$, defined by $\mathcal{D}^{(0)}=\mathcal{D}$ and $\mathcal{D}^{(i+1)}=\mathcal{D}^{(i)}+\left[\mathcal{D}^{(i)}, \mathcal{D}^{(i)}\right]$. Goursat structures appeared first in the work of von Weber and Cartan, who have shown that on an open and dense subset they can be converted into the so-called Goursat normal form. Later, Goursat structures have been studied by Kumpera and Ruiz. In the paper, we introduce a new local invariant for Goursat structures, called the singularity type, and prove that the growth vector and the abnormal curves of all elements of the derived flag are determined by this invariant. We provide a detailed analysis of all abnormal and rigid curves of Goursat structures. We show that neither abnormal curves, if $n \geq 6$, nor abnormal curves of all elements of the derived flag, if $n \geq 9$, determine the local equivalence class of a Goursat structure. The latter observation is deduced from a generalized version of Bäcklund's theorem. We also propose a new proof of a classical theorem of Kumpera and Ruiz. All results are illustrated by the $n$-trailer system, which, as we show, turns out to be a universal model for all local Goursat structures.
\end{abstract}

Résumé. Une structure de Goursat sur une variété de dimension $n$ est une distribution $\mathcal{D}$ de rang deux telle que $\operatorname{dim} \mathcal{D}^{(i)}=i+2$, pour $i=0, \ldots, n-2$, où les $\mathcal{D}^{(i)}$ sont les éléments du drapeau dérivé de $\mathcal{D}$, définis par $\mathcal{D}^{(0)}=\mathcal{D}$ et $\mathcal{D}^{(i+1)}=\mathcal{D}^{(i)}+\left[\mathcal{D}^{(i)}, \mathcal{D}^{(i)}\right]$. Les structures de Goursat sont d'abord apparues dans les travaux de von Weber et de Cartan, qui ont montré que sur un ouvert dense elles peuvent être transformées en la forme normale de Goursat. Ensuite, les structures de Goursat ont été étudiées par Kumpera et Ruiz. Dans cet article, nous introduisons un nouvel invariant local pour les structures des Goursat, appelé le type de singulatité, et montrons que le vecteur de croissance et les courbes anormales de tous les éléments du drapeau dérivé sont déterminés par cet invariant. Nous donnons une analyse détaillée de toutes les courbes anormales et rigides. Nous montrons que ni les courbes anormales, lorsque $n \geq 6$, ni les courbes anormales de tous les éléments du drapeau dérivé, lorsque $n \geq 9$, determinent la classe d'équivalence locale d'une structure de Goursat. Cette dernière observation est déduite d'une version généralisée du théorème de Bäcklund. Nous proposons aussi une nouvelle preuve d'un théorème classique de Kumpera et Ruiz. Tous nos résultats sont illustrés par le camion avec $n$ remorques, qui, comme nous le montrons, s'avère être un modèle universel pour toutes les structures de Goursat.

Mathematics Subject Classification. 58A30, 53C15, 93B29, 58A17.

Received November 15, 1999. Revised August 8, 2000.

Keywords and phrases: Goursat structures, Kumpera-Ruiz normal forms, abnormal curves, nonholonomic control systems, trailer systems.

${ }^{1}$ Laboratoire des Signaux et Systèmes, CNRS - Supélec - Université Paris-Sud XI, Plateau du Moulon, 91192 Gif-sur-Yvette, France; e-mail: pasillas@lss.supelec.fr

2 Laboratoire de Mathématiques de l'INSA, Institut National des Sciences Appliquées de Rouen, Place E. Blondel, 76130 Mont-Saint-Aignan, France; e-mail: wresp@lmi.insa-rouen.fr 


\section{Contents}

Introduction $\quad 121$

1. Goursat structures 124

1.1. Derived flag and Goursat structures 124

1.2. Examples of Goursat structures 124

1.3. Goursat normal form $\quad 125$

2. Kumpera-Ruiz's theorem 126

2.1. Kumpera-Ruiz normal forms 126

2.2. Kumpera-Ruiz's theorem 127

2.3. Low dimensional examples 128

3. The $N$-trailer system $r$

3.1. Definition of the $N$-trailer system 129

3.2. Conversion of the $N$-trailer system into a Kumpera-Ruiz normal form 130

3.3. Conversion of an arbitrary Goursat structure into the $N$-trailer system 132

4. Singularity type 132

4.1. Characteristic distributions 133

4.2. Jacquard's language 133

4.3. Definition of the singularity type 134

4.4. Low dimensional examples 135

4.5. Singularity type of Kumpera-Ruiz normal forms 135

4.6. Singularity type of the $N$-trailer system 138

5. Growth vector $\quad 140$

5.1. Lie flag and growth vector $\quad 140$

5.2. Growth vector of the $N$-trailer system 141

5.3. Growth vector of Goursat structures 142

5.4. Growth vector and singularity type 142

5.5. Computing the singularity type 144

6. Abnormal curves 145

6.1. Integral and abnormal curves $\quad 145$

6.2. Abnormal curves of Goursat structures 146

6.3. An illustrative example 148

6.4. Abnormal curves and singularity type 149

6.5. Rigid curves of Goursat structures $\quad 150$

6.6. Rigid curves of the $N$-trailer system 152

7. Contact transformations 153

7.1. A singular version of Bäcklund's theorem 153

7.2. Are Goursat structures locally determined by their abnormal curves? 157

Appendix A. Proof of Kumpera-Ruiz theorem 163

Appendix B. Proof of Theorem 6.2 (abnormal curves) 166

Appendix C. Weber's problem 171

Appendix D. Additional normal forms 173

Appendix E. Figures of low-dimensional trailer systems $\quad 175$

E.1. The unicycle and the car $\quad 175$

E.2. The two-trailer system 176

E.3. The three-trailer system 177

$\begin{array}{ll}\text { References } & 179\end{array}$ 


\section{INTRODUCTION}

Let $\mathcal{D}$ be a smooth rank $k$ distribution on a smooth manifold $M$, that is a map that assigns smoothly to each point $p$ in $M$ a linear subspace $\mathcal{D}(p) \subset T_{p} M$ of dimension $k$. The derived flag of $\mathcal{D}$ is the sequence defined by $\mathcal{D}^{(0)}=\mathcal{D}$ and $\mathcal{D}^{(i+1)}=\mathcal{D}^{(i)}+\left[\mathcal{D}^{(i)}, \mathcal{D}^{(i)}\right]$, for $i \geq 1$. A Goursat structure on a manifold $M$ of dimension $n \geq 3$ is a rank two distribution $\mathcal{D}$ such that, for $0 \leq i \leq n-2$, the elements of its derived flag satisfy $\operatorname{dim} \mathcal{D}^{(i)}(p)=i+2$, for each point $p$ in $M$. Goursat structures were introduced, using the dual language of Pfaffian systems, by von Weber in 1898. The first period of interest in this special class of distributions culminated in the work of Cartan and Goursat. A new period was initiated by Giaro, Kumpera, and Ruiz at the end of the seventies. A renewal of interest in Goursat structures has been observed from the mid of nineties.

There are at least three reasons explaining those one century long studies. The first reason is that any Goursat structure on $\mathbb{R}^{n}$ can be locally converted (on an open and dense subset, as it was observed only later by Giaro et al. [20]) into the so-called Goursat normal form, also known as chained form:

$$
\left(\frac{\partial}{\partial x_{n}}, x_{n} \frac{\partial}{\partial x_{n-1}}+\cdots+x_{3} \frac{\partial}{\partial x_{2}}+\frac{\partial}{\partial x_{1}}\right)
$$

It seems that von Weber [72] was the first to exhibit this property and, indeed, Goursat [22] attributes to him this result. In fact, the starting point of von Weber's studies was the following question: "when is a given distribution equivalent to Goursat normal form?", which had led him to discover the concept of derived flag. This question is very natural because Goursat normal form has a clear geometric interpretation. Indeed, let us consider the space $J^{k}(\mathbb{R}, \mathbb{R})$ of $k$-jets of maps from $\mathbb{R}$ to $\mathbb{R}$. On the one hand, a necessary condition for a curve in $J^{k}(\mathbb{R}, \mathbb{R})$ to be a prolongation of a graph of a function from $\mathbb{R}$ to $\mathbb{R}$ is that it is an integral curve of a distribution which, in the canonical coordinates of $J^{k}(\mathbb{R}, \mathbb{R})$, is spanned by the Goursat normal form on $\mathbb{R}^{k+2}$. On the other hand, a necessary and sufficient condition for a diffeomorphism of $J^{k}(\mathbb{R}, \mathbb{R})$ to map prolongations of graphs of functions into prolongations of graphs of functions is to preserve the distribution spanned by the Goursat normal form on $\mathbb{R}^{k+2}$. Such diffeomorphisms are called contact transformations [55] of order $k$ and have been intensively studied by Bäcklund [2], and by Lie and Scheffers [40].

The second reason of interest in Goursat structures has been the classical problem, first considered by Monge, of integrating underdetermined differential equations (equivalently, Pfaffian systems) without integration. To be more precise, let $\mathcal{D}$ be a rank $k$ distribution on $M$. The problem (see e.g. [21] and [74]) is to find $k$ smooth functions $\varphi_{1}, \ldots, \varphi_{k}$ such that any integral curve $\gamma(t)$ of $\mathcal{D}$ can be expressed as a smooth function of $\varphi_{1}, \ldots, \varphi_{k}$ and their time-derivatives along $\gamma(t)$. The most important achievement of the first period of studies on Goursat structures was a result of Cartan [11], who showed that a rank two distribution possesses the above described property if and only if it is transformable into Goursat normal form.

The third reason of importance of Goursat structures is that they describe the nonholonomic constraints of many mechanical systems. For example, the kinematical constraints of a passenger car are described by a Goursat structure on $\mathbb{R}^{2} \times\left(S^{1}\right)^{2}$; those of a truck by a Goursat structure on $\mathbb{R}^{2} \times\left(S^{1}\right)^{3}$. Moreover, for Goursat structures the nonholonomic motion planning problem can be solved explicitly; either by transforming them into Goursat normal form, as suggested by Murray and Sastry (see e.g. [53] and [54]), or by using the concept of flatness, introduced in control theory by Fliess, Lévine, Martin, and Rouchon, which is the above described property of calculating the trajectories without integration (see e.g. [43] and [17]).

As we said, the second period of studies on Goursat structures began with a work of Giaro et al. [20], who observed that there are Goursat structures which are not locally equivalent to Goursat normal form. This observation raised the problem of classification of Goursat structures and that of finding their invariants, and has led Kumpera and Ruiz to write their important paper [32], where they gave a complete classification, up to dimension 7 , together with a set of general results on Goursat structures.

In the nineties, research on Goursat structures was concentrated around two main topics: the classification problem and the nonholonomic motion planning problem for mechanical systems described by Goursat structures. Among results concerning the classification problem, Murray [53] obtained, using the concept of 
growth vector $^{1}$, an easily checkable necessary and sufficient condition for a Goursat structure to be equivalent to Goursat normal form (his condition simplifies those of Libermann [39] and Kumpera and Ruiz [32]). Gaspar [19] and, independently, Cheaito and Mormul [12] corrected the classification proposed by Kumpera and Ruiz in dimension 7. Complete classifications were then obtained in dimension 8, by Cheaito and Mormul [12], and in dimension 9, by Mormul [50]. It turns out that this dimension is the highest one in which there is a finite number of non-equivalent Goursat structures. Indeed, Cheaito et al. [13] showed that in higher dimensions there are real continuous parameters in the classification. Note, however, that in each dimension all Goursat structures are finitely determined, which implies that there are no functional parameters in the classification. Although new important results have been recently obtained by Mormul [52] and Montgomery and Zhitomirskiu [46], the general classification problem is still open in dimensions higher that 9 .

Most of the work concerning mechanical control systems described by Goursat structures has been motivated by the study of the $n$-trailer system. It would be impossible to give here a complete set of references on this subject. We have thus chosen to cite two books [36,38], and to give a few references concerning standard control theory problems for the $n$-trailer system and chained systems.

The controllability of the $n$-trailer system has been proved by Laumond both for regular [34] and singular [35] configurations. Improved bounds for the nonholonomy degree of the $n$-trailer at singular configurations have been obtained by Sørdalen [63], Luca and Risler [42], and Jean [29]. For regular configurations, an explicit conversion of the $n$-trailer system into chained form has been obtained by Sørdalen [62]; for singular configurations, an explicit conversion of the $n$-trailer system into Kumpera-Ruiz normal form has been obtained by the authors [57] (see also Sect. 3).

Open loop motion planning has been investigated for general nonholonomic systems by Brockett [4], Lafferriere and Sussmann [33], and Liu [41]. For chained systems, these results have been considerably simplified by Murray and Sastry [54] (using the special properties of chained form) and by Fliess et al. [17] (using the concept of flatness). Combined with the conversion of the $n$-trailer into chained form obtained by Sørdalen [62], they have led to a solution of the nonholonomic motion planning problem for the $n$-trailer system (see e.g. [37,60], and $[69])$.

Path tracking of non-abnormal trajectories has been studied by Fliess et al. [17], Jiang and Nijmeijer [30], and Walsh et al. [71]. Since for chained systems constant trajectories (points) are abnormal, the proposed path tracking strategies cannot be applied to achieve pointwise stabilization. Indeed, the linearization of a chained system around such trajectories is not controllable. The first who observed the difficulties of pointwise stabilization for control systems without drift was Brockett [5]. General algorithms for pointwise stabilization of nonholonomic systems can be found in the work of Coron [14], Pomet [58], McCloskey and Murray [44], and Morin and Samson [47]. For chained systems, improved results have been obtained by Samson [61], Sørdalen and Egeland [64], and Teel et al. [68]. These methods have been successfully applied to the $n$-trailer system (see e.g. Samson [61], Sørdalen and Wichlund [66], and the references given there).

Our paper is devoted to a study of the geometry of Goursat structures. Our first main contribution is to introduce a new local invariant for Goursat structures, called the singularity type, whose definition is based on the following observation, which goes back to Cartan [11] (compare [8,13,32,43,46], and Appendix C). If $\mathcal{D}$ is a Goursat structure then each element $\mathcal{D}^{(i)}$ of its derived flag contains an involutive subdistribution $\mathcal{C}_{i} \subset \mathcal{D}^{(i)}$ that has constant corank one in $\mathcal{D}^{(i)}$ and is characteristic for $\mathcal{D}^{(i+1)}$. The singularity type reflects the geometry of incidence between the distributions $\mathcal{D}^{(i)}$ and the distributions $\mathcal{C}_{i}$. Although, as we prove, the singularity type keeps the same information about a Goursat structure as the growth vector, that information is encoded in the singularity type in a much more systematic and, what is extremely important, in a much more geometric way. In particular, the geometric information contained in the singularity type enables us to describe completely all abnormal curves of all elements of the derived flag. This can be summarized in the following theorem, which is a combination of Theorem 5.6 and Theorem 6.3, and gives one of the main results of the paper.

\footnotetext{
${ }^{1}$ The Lie flag of a distribution $\mathcal{D}$ is the sequence defined by $\mathcal{D}_{0}=\mathcal{D}$ and $\mathcal{D}_{i+1}=\mathcal{D}_{i}+\left[\mathcal{D}_{0}, \mathcal{D}_{i}\right]$, for $i \geq 1$. The sequence $\left(\operatorname{dim} \mathcal{D}_{i}(p)\right)_{i \geq 0}$ is called the growth vector of $\mathcal{D}$ at $p$.
} 
Theorem 1. Let $\mathcal{D}$ and $\tilde{\mathcal{D}}$ be two Goursat structures defined on two manifolds $M$ and $\tilde{M}$, respectively, of dimension $n \geq 3$. Fix two points $p$ and $\widetilde{p}$ of $M$ and $\tilde{M}$, respectively. The three following conditions are equivalent:

(i) the singularity type of $\mathcal{D}$ at $p$ equals the singularity type of $\tilde{\mathcal{D}}$ at $\tilde{p}$;

(ii) the growth vector of $\mathcal{D}$ at $p$ equals the growth vector of $\tilde{\mathcal{D}}$ at $\tilde{p}$;

(iii) there exists a diffeomorphism $\varphi$, with $\tilde{p}=\varphi(p)$, between two small enough neighborhoods of $p$ and $\tilde{p}$ that transforms the abnormal curves of $\mathcal{D}^{(i)}$ into the abnormal curves of $\tilde{\mathcal{D}}^{(i)}$, for each $i \geq 0$.

A crucial example that we will use to illustrate our results on Goursat structures is the $n$-trailer system, that is a mobile robot (unicycle) towing $n$ trailers. We will explicitly show how to transform locally the $n$-trailer system into a Kumpera-Ruiz normal form, and we will prove a surprising result, which is the second main contribution of the paper, stating that any Goursat structure is locally equivalent to the $n$-trailer system around a well chosen point of its configuration space (after the paper had been submitted, we learnt that an alternative proof of this result has been proposed by Montgomery and Zhitomirskiı [46]). This property will enable us to use for any Goursat structure a deep result of Jean [29] devoted to singular configurations of the $n$-trailer system, in particular we will extend to all Goursat structures Jean's formula for the growth vector of the $n$-trailer system. In our work, the singularity type will replace the angles of the $n$-trailer system that appear in Jean's theorem. We will also calculate rigid curves of the $n$-trailer and give their natural mechanical interpretation: they correspond to motions that fix the positions of the centers of at least two trailers.

In the paper, we propose an inductive procedure of constructing Kumpera-Ruiz normal forms of Goursat structures based on two types of prolongations: regular and singular. This construction provides a systematic and unifying approach to many results of the paper. In particular, it will be used to show that any Goursat structure can be brought to a Kumpera-Ruiz normal form; to study generalized contact transformations, that is transformations which preserve Goursat structures; and to define the above mentioned transformations that transform locally the $n$-trailer system into a Kumpera-Ruiz normal form, and, conversely, that convert locally an arbitrary Goursat structure into the $n$-trailer system around a well chosen point of its configuration space.

Recent studies (see [26] and [45]) show that most distributions are determined by their abnormal curves. Our complete description of abnormal curves of Goursat structures enables us to conclude that this is not the case for Goursat structures. Indeed, combining our study with the main theorem of [13] leads us to the following result, which is the third main contribution of the paper: neither abnormal curves, if $n \geq 6$, nor abnormal curves of all elements of the derived flag, if $n \geq 9$, determine the local equivalence class of a Goursat structure (see Prop. 7.4 and Prop. 7.5). The latter observation is deduced from Theorem 7.3, which provides a generalized version of Bäcklund's theorem. In other words, the singularity type (equivalently, the growth vector) determines completely the collection of all abnormal curves of a Goursat structure but does not characterize the collection of all integral curves.

The paper is organized as follows. In Section 1, we introduce Goursat structures, we give some examples in small dimension, and we define Goursat normal form. In Section 2, we provide an inductive definition of Kumpera-Ruiz normal form. The proposed concept of prolongations enables us to give a new proof of the Kumpera-Ruiz theorem (presented in Appendix A), which states that any Goursat structure can be converted locally into a Kumpera-Ruiz normal form. In Section 3, we introduce the $n$-trailer system and we construct transformations that bring locally the $n$-trailer system into a Kumpera-Ruiz normal form and, conversely, that bring an arbitrary Goursat structure into the $n$-trailer system. In Section 4 , we introduce our main invariant of Goursat structures, namely, the singularity type. We also compute the singularity type for Kumpera-Ruiz normal forms and for the $n$-trailer system. As we have said, the singularity type keeps the same information about Goursat structures as the growth vector although in both invariants that information is encoded in a different way. Section 5 is devoted to study relations between these two invariants. In particular, we give a formula to compute the growth vector of an arbitrary Goursat structure and another to compute the singularity type using the growth vector. In Section 6 we study abnormal curves of Goursat structures. We give a complete description of absolutely continuous abnormal curves for all elements of the derived flag of any Goursat structure. 
We prove that the whole information about all abnormal curves is given by the singularity type. In Section 7 we study generalized contact transformations, that is transformations which preserve Goursat structures (also called symmetries) and we give formulas to calculate them starting from first order contract transformations. Those formulas are used to analyze examples of Goursat structures that are non-equivalent but that have diffeomorphic abnormal curves.

In Appendix A, we give our proof of the Kumpera-Ruiz theorem. Appendix B contains proofs of results that describe abnormal curves. Appendix $\mathrm{C}$ is devoted to a class of distributions that, although of rank greater than two, are very close to Goursat structures. This class was initially studied by von Weber. In Appendix D, we construct a normal form that we use in our study of rigidity of integral curves of Goursat structures. Finally, in Appendix E, we illustrate through a set of figures different configurations of the $n$-trailer system corresponding to various Kumpera-Ruiz normal forms in dimensions 3, 4, 5, and 6 .

The authors would like to thank an anonymous reviewer for his comments, which helped to improve the presentation.

\section{Goursat structures}

In this section we introduce Goursat structures, the main object of our study, which form a particular class of rank two distributions. In the successive subsections we, respectively, define Goursat structures, give simple examples, and introduce Goursat normal form, which serves as a canonical form for any Goursat structure at a typical point.

\subsection{Derived flag and Goursat structures}

A rank $k$ distribution $\mathcal{D}$ on a smooth manifold $M$ is a map that assigns smoothly to each point $p$ in $M$ a linear subspace $\mathcal{D}(p) \subset T_{p} M$ of dimension $k$. In other words, a rank $k$ distribution is a smooth rank $k$ subbundle of the tangent bundle $T M$. Such a field of tangent $k$-planes is spanned locally by $k$ pointwise linearly independent smooth vector fields $f_{1}, \ldots, f_{k}$ on $M$, which will be denoted by $\mathcal{D}=\left(f_{1}, \ldots, f_{k}\right)$.

Two distributions $\mathcal{D}$ and $\tilde{\mathcal{D}}$ defined on two manifolds $M$ and $\tilde{M}$, respectively, are equivalent if there exists a smooth diffeomorphism $\varphi$ between $M$ and $\tilde{M}$ such that

$$
\left(\varphi_{*} \mathcal{D}\right)(\tilde{p})=\tilde{\mathcal{D}}(\tilde{p})
$$

for each point $\tilde{p}$ in $\tilde{M}$. They are locally equivalent at two points $p$ and $\tilde{p}$ that belong to $M$ and $\tilde{M}$, respectively, if there exists two small enough neighborhoods $U$ and $\tilde{U}$ of $p$ and $\tilde{p}$, respectively, and a diffeomorphism $\varphi$ between $U$ and $\tilde{U}$ such that $\varphi(p)=\tilde{p}$ and $\left(\varphi_{*} \mathcal{D}\right)(\tilde{p})=\tilde{\mathcal{D}}(\tilde{p})$, for each point $\tilde{p}$ in $\tilde{U}$.

The derived flag of a distribution $\mathcal{D}$ is the sequence $\mathcal{D}^{(0)} \subset \mathcal{D}^{(1)} \subset \cdots$ defined inductively by

$$
\mathcal{D}^{(0)}=\mathcal{D} \quad \text { and } \quad \mathcal{D}^{(i+1)}=\mathcal{D}^{(i)}+\left[\mathcal{D}^{(i)}, \mathcal{D}^{(i)}\right], \quad \text { for } i \geq 0 .
$$

This sequence should not be confused with the Lie flag (33), which will be introduced in Section 5.1.

Definition 1.1. A Goursat structure on a manifold $M$ of dimension $n \geq 3$ is a rank two distribution $\mathcal{D}$ such that, for $0 \leq i \leq n-2$, the elements of its derived flag satisfy $\operatorname{dim} \mathcal{D}^{(i)}(p)=i+2$, for each point $p$ in $M$.

\subsection{Examples of Goursat structures}

A Goursat structure on a three-manifold is a contact structure, and is locally equivalent to the distribution spanned by

$$
\left(\frac{\partial}{\partial x_{3}}, x_{3} \frac{\partial}{\partial x_{2}}+\frac{\partial}{\partial x_{1}}\right)
$$


which is called Pfaff-Darboux normal form. A Goursat structure on a four-manifold is an Engel structure, and is locally equivalent to the distribution spanned by

$$
\left(\frac{\partial}{\partial x_{4}}, x_{4} \frac{\partial}{\partial x_{3}}+x_{3} \frac{\partial}{\partial x_{2}}+\frac{\partial}{\partial x_{1}}\right)
$$

which is called Engel normal form. Observe that, for a generic field of planes $\mathcal{D}$ on $\mathbb{R}^{3}$, we have $\operatorname{dim} \mathcal{D}^{(1)}(p)=3$, for any point $p$ on an open and dense subset of $\mathbb{R}^{3}$; for a generic field of planes $\mathcal{D}$ on $\mathbb{R}^{4}$, we have $\operatorname{dim} \mathcal{D}^{(1)}(p)=3$ and $\operatorname{dim} \mathcal{D}^{(2)}(p)=4$, for any point $p$ on an open and dense subset of $\mathbb{R}^{4}$. Therefore, in a small enough neighborhood of a typical point, a generic field of planes on a manifold of dimension three or four is a Goursat structure. Note, however, that starting from dimension five the class of Goursat structures is of infinite codimension within the class of all rank two distributions. Indeed, for a generic field of planes $\mathcal{D}$ on $\mathbb{R}^{n}$, for $n \geq 5$, we have $\operatorname{dim} \mathcal{D}^{(1)}(p)=3$ and $\operatorname{dim} \mathcal{D}^{(2)}(p)=5$, for any point $p$ on an open and dense subset of $\mathbb{R}^{n}$.

We give now a mechanical example. For $n \geq 0$, the distribution spanned by the following pair of vector fields:

$$
\left(\frac{\partial}{\partial \theta_{n}}, \cos \left(\theta_{0}\right) \pi_{0} \frac{\partial}{\partial \xi_{1}}+\sin \left(\theta_{0}\right) \pi_{0} \frac{\partial}{\partial \xi_{2}}+\sum_{i=0}^{n-1} \sin \left(\theta_{i+1}\right) \pi_{i+1} \frac{\partial}{\partial \theta_{i}}\right),
$$

where $\pi_{i}=\prod_{j=i+1}^{n} \cos \left(\theta_{j}\right)$ and $\pi_{n}=1$, is a Goursat structure on $\mathbb{R}^{2} \times\left(S^{1}\right)^{n+1}$ equipped with coordinates $\left(\xi_{1}, \xi_{2}, \theta_{0}, \ldots, \theta_{n}\right)$. This distribution is the kinematical model for the "nonholonomic manipulator" of Sørdalen et al. [65]. Another example is the $n$-trailer system (see Sect. 3), which will play a fundamental role in this article.

\subsection{Goursat normal form}

The concepts of derived flag and Goursat structure were introduced, using the dual language of Pfaffian systems, by von Weber [72] in order to characterize the class of Pfaffian systems that can be converted into a particular normal form, also introduced by him, which is now known as Goursat normal form (see (3) below; see also Appendix C).

Although it is not clear who was the first to prove that Goursat structures can be converted locally into Goursat normal form, at least on an open and dense subset ([72], Th. VI), compare [6,11,22,32, 53, 72]; it is clear that the foundations of this result were set by Engel and von Weber (see $[9,16,72]$, and the references given there; see also Appendix C). The importance of this result was, however, fully understood only later, by Cartan when he solved a long standing problem of that time: the characterization of explicitly integrable Monge equations [11] (see also [6, 20, 22, 23, 43,74]).

Theorem 1.2 (von Weber-Cartan-Goursat). Let $\mathcal{D}$ be a Goursat structure defined on a manifold $M$ of dimension $n \geq 3$. There exists an open and dense subset $U \subset M$ such that, for any point $p$ in $U$, the distribution $\mathcal{D}$ is locally equivalent at $p$ to the distribution spanned by the following pair of vector fields:

$$
\left(\frac{\partial}{\partial x_{n}}, x_{n} \frac{\partial}{\partial x_{n-1}}+x_{n-1} \frac{\partial}{\partial x_{n-2}}+\cdots+x_{3} \frac{\partial}{\partial x_{2}}+\frac{\partial}{\partial x_{1}}\right),
$$

considered on a small enough neighborhood $V \subset \mathbb{R}^{n}$ of zero. Moreover, if $n=3$ or 4 then $U=M$.

In control theory, the normal form (3) is usually called chained form. In order to keep the classical name, we will call it Goursat normal form. An elegant characterization, using the growth vector, of the above mentioned open and dense set $U$ was obtained by Murray [53] (see Th. 5.2). Observe that, in most of the above mentioned references, Goursat structures are not defined by distributions but by their duals, that is by Pfaffian systems. Note also that many other names have been given to Goursat structures: "systeme vom Charakter eins und vom Rang zwei" [72], "systèmes de classe zéro" [11], "systèmes spéciaux" [22], "systèmes en drapeaux" [32], "systems of Goursat type" [7], and "systems that satisfy the Goursat condition" [50]. 


\section{Kumpera-Ruiz's THEOREM}

In this section we introduce Kumpera-Ruiz normal forms, which are normal forms for Goursat structures, at any (regular or singular) point, that generalize Goursat normal form, defined in Section 1. In Section 2.1, we propose an inductive definition of Kumpera-Ruiz normal forms using the notion of (regular and singular) prolongations of vector fields. In Section 2.2, we recall a theorem of Kumpera and Ruiz, which states that any Goursat structure is locally equivalent to a Kumpera-Ruiz normal form. A new proof of that theorem, based on the notion of prolongation of vector fields, is given in Appendix A. In Section 2.3, we give examples of Kumpera-Ruiz normal forms in dimensions five and six and very briefly discuss the classification problem of Goursat structures.

\subsection{Kumpera-Ruiz normal forms}

If at a given point a Goursat structure can be converted into Goursat normal form then this point is called regular; otherwise, it is called singular. The first who observed the existence of singular points were Giaro et al. [20]. This initial observation has led Kumpera and Ruiz to write their pioneering paper [32], where they introduced the normal forms that we will consider in this section.

We start with the Pfaff-Darboux and Engel normal forms, given respectively on $\mathbb{R}^{3}$, equipped with coordinates $\left(x_{1}, x_{2}, x_{3}\right)$, and $\mathbb{R}^{4}$, equipped with coordinates $\left(x_{1}, x_{2}, x_{3}, x_{4}\right)$, by the pairs of vector fields $\kappa^{3}=\left(\kappa_{1}^{3}, \kappa_{2}^{3}\right)$ and $\kappa^{4}=\left(\kappa_{1}^{4}, \kappa_{2}^{4}\right)$, defined by

$$
\begin{aligned}
\kappa_{1}^{3} & =\frac{\partial}{\partial x_{3}} \\
\kappa_{2}^{3} & =x_{3} \frac{\partial}{\partial x_{2}}+\frac{\partial}{\partial x_{1}}
\end{aligned}
$$

and

$$
\begin{aligned}
\kappa_{1}^{4} & =\frac{\partial}{\partial x_{4}} \\
\kappa_{2}^{4} & =x_{4} \frac{\partial}{\partial x_{3}}+x_{3} \frac{\partial}{\partial x_{2}}+\frac{\partial}{\partial x_{1}} .
\end{aligned}
$$

Loosely speaking, we can write

$$
\begin{aligned}
\kappa_{1}^{4} & =\frac{\partial}{\partial x_{4}} \\
\kappa_{2}^{4} & =x_{4} \kappa_{1}^{3}+\kappa_{2}^{3} .
\end{aligned}
$$

In order to make this precise we will adopt the following natural notation. Consider a vector field

$$
f^{n-1}=\sum_{i=1}^{n-1} f_{i}^{n-1}\left(x_{1}, \ldots, x_{n-1}\right) \frac{\partial}{\partial x_{i}}
$$

on $\mathbb{R}^{n-1}$ equipped with coordinates $\left(x_{1}, \ldots, x_{n-1}\right)$. We can lift $f^{n-1}$ to a vector field, denoted also by $f^{n-1}$, on $\mathbb{R}^{n}$ equipped with coordinates $\left(x_{1}, \ldots, x_{n-1}, x_{n}\right)$ by taking

$$
f^{n-1}=\sum_{i=1}^{n-1} f_{i}^{n-1}\left(x_{1}, \ldots, x_{n-1}\right) \frac{\partial}{\partial x_{i}}+0 \cdot \frac{\partial}{\partial x_{n}} .
$$

That is, we lift $f^{n-1}$ by translating it along the $x_{n}$-direction. 
Notation 2.1. From now on, in any expression of the form $\kappa_{2}^{n}=\alpha(x) \kappa_{1}^{n-1}+\beta(x) \kappa_{2}^{n-1}$, the vector fields $\kappa_{1}^{n-1}$ and $\kappa_{2}^{n-1}$ should be considered as the above defined lifts of $\kappa_{1}^{n-1}$ and $\kappa_{2}^{n-1}$, respectively.

Let $\kappa^{n-1}=\left(\kappa_{1}^{n-1}, \kappa_{2}^{n-1}\right)$ denote a pair of vector fields on $\mathbb{R}^{n-1}$. A regular prolongation, with parameter $c_{n}$, of $\kappa^{n-1}$, denoted by $\kappa^{n}=R_{c_{n}}\left(\kappa^{n-1}\right)$, is a pair of vector fields $\kappa^{n}=\left(\kappa_{1}^{n}, \kappa_{2}^{n}\right)$ defined on $\mathbb{R}^{n}$ by

$$
\begin{aligned}
\kappa_{1}^{n} & =\frac{\partial}{\partial x_{n}} \\
\kappa_{2}^{n} & =\left(x_{n}+c_{n}\right) \kappa_{1}^{n-1}+\kappa_{2}^{n-1},
\end{aligned}
$$

where $c_{n}$ belongs to $\mathbb{R}$. The singular prolongation of $\kappa^{n-1}$, denoted by $\kappa^{n}=S\left(\kappa^{n-1}\right)$, is the pair of vector fields $\kappa^{n}=\left(\kappa_{1}^{n}, \kappa_{2}^{n}\right)$ defined on $\mathbb{R}^{n}$ by

$$
\begin{aligned}
\kappa_{1}^{n} & =\frac{\partial}{\partial x_{n}} \\
\kappa_{2}^{n} & =\kappa_{1}^{n-1}+x_{n} \kappa_{2}^{n-1} .
\end{aligned}
$$

Definition 2.2. A pair of vector fields $\kappa^{n}$ on $\mathbb{R}^{n}$, for $n \geq 3$, is called a Kumpera-Ruiz normal form if $\kappa^{n}=$ $\sigma_{n-3} \circ \cdots \circ \sigma_{1}\left(\kappa^{3}\right)$, where each $\sigma_{i}$, for $1 \leq i \leq n-3$, equals either $S$ or $R_{c_{i}}$, for some real constants $c_{i}$.

We will also call a Kumpera-Ruiz normal form the restriction of a Kumpera-Ruiz normal form to any open subset of $\mathbb{R}^{n}$. Most often, the coordinates $x_{1}, \ldots, x_{n}$ will be the elements of a coordinate chart $x: M \rightarrow \mathbb{R}^{n}$, defined in a neighborhood of a given point $p$ in $M$. If we have $x(p)=0$ then we will say that the Kumpera-Ruiz normal form is centered at $p$. For example, the two models considered in [20]:

$$
\begin{aligned}
& \left(\frac{\partial}{\partial x_{5}}, x_{5} \frac{\partial}{\partial x_{4}}+x_{4} \frac{\partial}{\partial x_{3}}+x_{3} \frac{\partial}{\partial x_{2}}+\frac{\partial}{\partial x_{1}}\right) \\
& \left(\frac{\partial}{\partial x_{5}}, \frac{\partial}{\partial x_{4}}+x_{5}\left(x_{4} \frac{\partial}{\partial x_{3}}+x_{3} \frac{\partial}{\partial x_{2}}+\frac{\partial}{\partial x_{1}}\right)\right),
\end{aligned}
$$

defined by $R_{0}\left(\kappa^{4}\right)$ and $S\left(\kappa^{4}\right)$, respectively, are Kumpera-Ruiz normal forms on $\mathbb{R}^{5}$, equipped with coordinates $\left(x_{1}, \ldots, x_{5}\right)$, centered at zero.

\subsection{Kumpera-Ruiz's theorem}

The following theorem of Kumpera and Ruiz shows clearly the importance of their normal forms. We will prove this theorem in Appendix A because many of our results are based on a construction that also appears in our proof. Moreover, we would like to emphasize two features of our proof. Firstly, it is quite close to the original ideas of von Weber. Indeed, though we use distributions instead of Pfaffian systems, the two proofs share the same fundamental lemma (compare [72], Th. V and Prop. A.1; see also Appendix C). Secondly, it is to our knowledge the only one that does not mix the language of vector fields and differential forms (everything is done in terms of vector fields). For alternative proofs we refer the reader to [12] and to the original work of Kumpera and Ruiz [32].

Theorem 2.3 (Kumpera-Ruiz). Any Goursat structure on a manifold $M$ of dimension $n$ is locally equivalent, at any point $p$ in $M$, to a distribution spanned by a Kumpera-Ruiz normal form centered at $p$ and defined on a suitably chosen neighborhood $U \subset \mathbb{R}^{n}$ of zero.

This theorem is the cornerstone to understand the geometry of Goursat structures. On the one hand, this result implies that locally, even at singular points, Goursat structures do not have functional invariants; this property makes them precious but rare and distinguishes them from generic rank two distributions on $n$-manifolds, which do have local functional invariants when $n \geq 5$ (see $[10,27,70,78]$ ). But on the other hand, 
the real constants that appear in Kumpera-Ruiz normal forms are unavoidable; this fact has been observed only recently and implies that Goursat structures do have real invariants (see [13,50], and Sect. 7).

Though our definition of Kumpera-Ruiz normal forms was inductive, it is possible to give the following equivalent explicit definition (6), which will also be used in the paper. Observe that in the normal form (6), we use a double indexation $x_{j}^{i}$ of coordinates, for $0 \leq i \leq m+1$, where the integer $m$ gives the number of singularities of the normal form, that is the number of singular prolongations (provided that $\sigma_{1}$ is regular, which can always be assumed without lose of generality).

Corollary 2.4. Any Goursat structure defined on a manifold $M$ of dimension $n \geq 4$ is locally equivalent, at any point $p$ in $M$, to a distribution spanned in a small neighborhood of zero by a pair of vector fields that has the following form:

$$
\left(\frac{\partial}{\partial x_{1}^{0}}, \sum_{i=0}^{m}\left(\prod_{j=0}^{i-1} x_{k_{j}}^{j}\right)\left(\sum_{j=1}^{k_{i}-1}\left(x_{j}^{i}+c_{j}^{i}\right) \frac{\partial}{\partial x_{j+1}^{i}}+\frac{\partial}{\partial x_{1}^{i+1}}\right)\right),
$$

where the coordinates $x_{j}^{i}$, for $0 \leq i \leq m+1$ and $1 \leq j \leq k_{i}$, are centered at $p$; the integer $m$ is such that $0 \leq m \leq n-4 ;$ and the integers $k_{i}$, for $0 \leq i \leq m+1$, satisfy $k_{0} \geq 1, \ldots, k_{m-1} \geq 1, k_{m} \geq 3, k_{m+1}=1$ and $\sum_{i=0}^{m+1} k_{i}=n$. The constants $c_{j}^{i}$, for $1 \leq j \leq k_{i}-1$, are real constants.

\subsection{Low dimensional examples}

Let us recall the complete local classification of Goursat structures on manifolds of dimension five and six, obtained by Giaro et al. (see [20] and [32]).

(i) Any Goursat structure on a five-manifold is locally equivalent to one of the two following Kumpera-Ruiz normal forms

$$
\begin{aligned}
& \left(\frac{\partial}{\partial x_{5}}, x_{5} \frac{\partial}{\partial x_{4}}+x_{4} \frac{\partial}{\partial x_{3}}+x_{3} \frac{\partial}{\partial x_{2}}+\frac{\partial}{\partial x_{1}}\right) \\
& \left(\frac{\partial}{\partial x_{5}}, \frac{\partial}{\partial x_{4}}+x_{5}\left(x_{4} \frac{\partial}{\partial x_{3}}+x_{3} \frac{\partial}{\partial x_{2}}+\frac{\partial}{\partial x_{1}}\right)\right),
\end{aligned}
$$

which are not locally equivalent at zero.

(ii) Any Goursat structure on a six-manifold is locally equivalent to one of the five following Kumpera-Ruiz normal forms

$$
\begin{aligned}
& \left(\frac{\partial}{\partial x_{6}}, x_{6} \frac{\partial}{\partial x_{5}}+x_{5} \frac{\partial}{\partial x_{4}}+x_{4} \frac{\partial}{\partial x_{3}}+x_{3} \frac{\partial}{\partial x_{2}}+\frac{\partial}{\partial x_{1}}\right) \\
& \left(\frac{\partial}{\partial x_{6}}, \frac{\partial}{\partial x_{5}}+x_{6}\left(x_{5} \frac{\partial}{\partial x_{4}}+x_{4} \frac{\partial}{\partial x_{3}}+x_{3} \frac{\partial}{\partial x_{2}}+\frac{\partial}{\partial x_{1}}\right)\right) \\
& \left(\frac{\partial}{\partial x_{6}}, x_{6} \frac{\partial}{\partial x_{5}}+\frac{\partial}{\partial x_{4}}+x_{5}\left(x_{4} \frac{\partial}{\partial x_{3}}+x_{3} \frac{\partial}{\partial x_{2}}+\frac{\partial}{\partial x_{1}}\right)\right) \\
& \left(\frac{\partial}{\partial x_{6}},\left(x_{6}+1\right) \frac{\partial}{\partial x_{5}}+\frac{\partial}{\partial x_{4}}+x_{5}\left(x_{4} \frac{\partial}{\partial x_{3}}+x_{3} \frac{\partial}{\partial x_{2}}+\frac{\partial}{\partial x_{1}}\right)\right) \\
& \left(\frac{\partial}{\partial x_{6}}, \frac{\partial}{\partial x_{5}}+x_{6}\left(\frac{\partial}{\partial x_{4}}+x_{5}\left(x_{4} \frac{\partial}{\partial x_{3}}+x_{3} \frac{\partial}{\partial x_{2}}+\frac{\partial}{\partial x_{1}}\right)\right)\right),
\end{aligned}
$$

which are pairwise locally non-equivalent at zero. Observe that these two results are not implied by Theorem 2.3. Indeed, they show that in dimension five and six the constants that appear in Kumpera-Ruiz's theorem can be "normalized" to either 0 or 1 . 
For $n=7,8$ and 9 the complete classification is more delicate, but there is still a finite number of models (see [12,19,32], and [50]). For $n \geq 10$, the number of local models is infinite (see [13,50], and Sect. 7) and the complete classification remains an open problem (see recent results in [51]).

\section{THE $N$-TRAILER SYSTEM}

This section is devoted to the $n$-trailer system, which will be a crucial example in our study of Goursat structures. This system is composed of a mobile robot that tows $n$ passive trailers. Since the $n$-trailer system is a Goursat structure, it can be transformed locally into Goursat normal form, at regular points, and into KumperaRuiz normal form, at singular points. In Section 3.2, we provide an explicit construction of transformations that bring locally the $n$-trailer system into a Kumpera-Ruiz normal form. In Section 3.3, we prove a surprising result, which is one of the main contributions of the paper, stating that any Goursat structure is locally equivalent to the $n$-trailer system around a well chosen point of its configuration space.

\subsection{Definition of the $N$-trailer system}

The kinematical model for a unicycle-like mobile robot towing $n$ trailers such that the tow hook of each trailer is located at the center of its unique axle is usually called, in control theory, the $n$-trailer system (see the books [36] and [38]; the papers $[17,25,29,34,60-62,68,69]$ and the references given there). Figures representing this system are given in Appendix E. For simplicity, we will assume that the distances between any two consecutive trailers are equal.

Definition 3.1. The $n$-trailer system is the distribution defined on $\mathbb{R}^{2} \times\left(S^{1}\right)^{n+1}$, for $n \geq 0$, by the following pair of vector fields:

$$
\left(\frac{\partial}{\partial \theta_{n}}, \pi_{0} \cos \left(\theta_{0}\right) \frac{\partial}{\partial \xi_{1}}+\pi_{0} \sin \left(\theta_{0}\right) \frac{\partial}{\partial \xi_{2}}+\sum_{i=0}^{n-1} \pi_{i+1} \sin \left(\theta_{i+1}-\theta_{i}\right) \frac{\partial}{\partial \theta_{i}}\right),
$$

where $\pi_{i}=\prod_{j=i+1}^{n} \cos \left(\theta_{j}-\theta_{j-1}\right)$ and $\pi_{n}=1$.

In the above definition, the functions $\xi_{1}, \xi_{2}, \theta_{0}, \ldots, \theta_{n}$ are coordinates on the manifold $\mathbb{R}^{2} \times\left(S^{1}\right)^{n+1}$. The coordinates $\xi_{1}$ and $\xi_{2}$ represent the position of the last trailer, while the coordinates $\theta_{0}, \ldots, \theta_{n}$ represent, starting from the last trailer, the angles between each trailer's axle and the $\xi_{1}$-axis. It is easy to check that the $n$-trailer system is a Goursat structure (see e.g. [35]).

We give now an equivalent inductive definition of the $n$-trailer. This definition already appears in [29] and reminds the one given in the previous section for Kumpera-Ruiz normal forms. To start with, consider the pair of vector fields $\left(\tau_{1}^{0}, \tau_{2}^{0}\right)$ on $\mathbb{R}^{2} \times S^{1}$ that describe the kinematics of the unicycle-like mobile robot towing no trailers:

$$
\begin{aligned}
\tau_{1}^{0} & =\frac{\partial}{\partial \theta_{0}} \\
\tau_{2}^{0} & =\cos \left(\theta_{0}\right) \frac{\partial}{\partial \xi_{1}}+\sin \left(\theta_{0}\right) \frac{\partial}{\partial \xi_{2}} .
\end{aligned}
$$

The $n$-trailer system can be defined by applying successively a sequence of prolongations to this mobile robot. In order to do this, suppose that a pair of vector fields $\tau^{n-1}=\left(\tau_{1}^{n-1}, \tau_{2}^{n-1}\right)$ on $\mathbb{R}^{2} \times\left(S^{1}\right)^{n}$, associated to the mobile robot towing $n-1$ trailers, has been defined. The pair of vector fields $\tau^{n}=\left(\tau_{1}^{n}, \tau_{2}^{n}\right)$ on $\mathbb{R}^{2} \times\left(S^{1}\right)^{n+1}$ corresponding to the $n$-trailer system is then given by

$$
\begin{aligned}
\tau_{1}^{n} & =\frac{\partial}{\partial \theta_{n}} \\
\tau_{2}^{n} & =\sin \left(\theta_{n}-\theta_{n-1}\right) \tau_{1}^{n-1}+\cos \left(\theta_{n}-\theta_{n-1}\right) \tau_{2}^{n-1} .
\end{aligned}
$$


Observe that this definition should be understood in the sense of Notation 2.1, and that the pair of vector fields $\left(\tau_{1}^{n}, \tau_{2}^{n}\right)$ coincides with that of Definition 3.1. Mechanically, to prolongate the $n$-trailer means to add one more trailer to the system.

\subsection{Conversion of the $N$-trailer system into a Kumpera-Ruiz normal form}

Since the $n$-trailer is a Goursat structure, it follows directly from Kumpera-Ruiz's theorem that, in a small enough neighborhood of any point of its configuration space, in particular at any singular configuration, the $n$-trailer can be converted into Kumpera-Ruiz normal form. In this section we describe this conversion explicitly. For regular configurations, our result gives the transformations proposed in [62] and [69]; for singular configurations, our result gives a new kind of transformations.

Denote by $\zeta$ the coordinates of $\mathbb{R}^{2} \times\left(S^{1}\right)^{n+1}$, that is

$$
\zeta=\left(\zeta_{1}, \ldots, \zeta_{n+3}\right)=\left(\xi_{1}, \xi_{2}, \theta_{0}, \ldots, \theta_{n}\right)
$$

Fix a point $p$ of $\mathbb{R}^{2} \times\left(S^{1}\right)^{n+1}$ given in $\zeta$-coordinates by $\zeta(p)=\zeta^{p}=\left(\xi_{1}^{p}, \xi_{2}^{p}, \theta_{0}^{p}, \ldots, \theta_{n}^{p}\right)$. In order to convert, locally at $p$, the $n$-trailer into a Kumpera-Ruiz normal form we look for a local change of coordinates

$$
\left(x_{1}, \ldots, x_{n+3}\right)=\phi^{n}\left(\xi_{1}, \xi_{2}, \theta_{0}, \ldots, \theta_{n}\right),
$$

a Kumpera-Ruiz normal form $\left(\kappa_{1}^{n+3}, \kappa_{2}^{n+3}\right)$ on $\mathbb{R}^{n+3}$, and three smooth functions $\nu_{n}, \eta_{n}$, and $\mu_{n}$ such that

$$
\begin{aligned}
& \phi_{*}^{n}\left(\tau_{1}^{n}\right)=\left(\nu_{n} \circ \psi^{n}\right) \kappa_{1}^{n+3} \\
& \phi_{*}^{n}\left(\tau_{2}^{n}\right)=\left(\eta_{n} \circ \psi^{n}\right) \kappa_{1}^{n+3}+\left(\mu_{n} \circ \psi^{n}\right) \kappa_{2}^{n+3},
\end{aligned}
$$

where $\psi^{n}=\left(\phi^{n}\right)^{-1}$ denotes the inverse of the local diffeomorphism $\phi^{n}$ and both $\nu_{n}\left(\zeta^{p}\right) \neq 0$ and $\mu_{n}\left(\zeta^{p}\right) \neq 0$. Observe that we do not demand the $x$-coordinates to be centered at $p$, and thus the point $x(p)=\left(\phi^{n} \circ \zeta\right)(p)$ will be, in general, different from zero.

We construct inductively here a change of coordinates $\phi^{n}=\left(\phi_{1}, \ldots, \phi_{n+3}\right)^{T}$ and three smooth functions $\nu_{n}$, $\eta_{n}$, and $\mu_{n}$ that satisfy (15). We start with $n=0$. If $\theta_{0}^{p} \neq \pm \pi / 2 \bmod 2 \pi$ then define $\phi_{1}=\xi_{1}, \phi_{2}=\xi_{2}$, and $\phi_{3}=\tan \left(\theta_{0}\right)$. Moreover, take $\mu_{0}=\cos \left(\theta_{0}\right), \nu_{0}=\sec ^{2}\left(\theta_{0}\right)$, and $\eta_{0}=0$. If $\theta_{0}^{p}= \pm \pi / 2 \bmod 2 \pi$ then define $\phi_{1}=\xi_{2}$, $\phi_{2}=\xi_{1}$, and $\phi_{3}=\cot \left(\theta_{0}\right)$. Moreover, take $\mu_{0}=\sin \left(\theta_{0}\right), \nu_{0}=-\csc ^{2}\left(\theta_{0}\right)$, and $\eta_{0}=0$. Denote $s_{i}=\sin \left(\theta_{i}-\theta_{i-1}\right)$ and $c_{i}=\cos \left(\theta_{i}-\theta_{i-1}\right)$, for $0 \leq i \leq n$.

Now, consider the sequence of smooth functions defined locally around the point $\zeta(p)$, for $1 \leq i \leq n$, by either

$$
\begin{aligned}
\phi_{i+3} & =\frac{s_{i} \nu_{i-1}+c_{i} \eta_{i-1}}{c_{i} \mu_{i-1}} \\
\mu_{i} & =c_{i} \mu_{i-1} \\
\nu_{i} & =\mathrm{L}_{\tau_{1}^{i}} \phi_{i+3} \\
\eta_{i} & =\mathrm{L}_{\tau_{2}^{i}} \phi_{i+3},
\end{aligned}
$$

if $\theta_{i}^{p}-\theta_{i-1}^{p} \neq \pm \pi / 2 \bmod 2 \pi$ (regular case) or by

$$
\begin{aligned}
\phi_{i+3} & =\frac{c_{i} \mu_{i-1}}{s_{i} \nu_{i-1}+c_{i} \eta_{i-1}} \\
\mu_{i} & =s_{i} \nu_{i-1}+c_{i} \eta_{i-1} \\
\nu_{i} & =\mathrm{L}_{\tau_{1}^{i}} \phi_{i+3} \\
\eta_{i} & =\mathrm{L}_{\tau_{2}^{i}} \phi_{i+3},
\end{aligned}
$$


if $\theta_{i}^{p}-\theta_{i-1}^{p}= \pm \pi / 2 \bmod 2 \pi$ (singular case). It is easy to prove that, for $0 \leq i \leq n$, the transformations defined by $\left(x_{1}, \ldots, x_{i+3}\right)=\phi^{i}\left(\xi_{1}, \xi_{2}, \theta_{0}, \ldots, \theta_{i}\right)$ are smooth changes of coordinates around $p_{i}$ and that, moreover, we have both $\nu_{i}\left(\zeta^{p_{i}}\right) \neq 0$ and $\mu_{i}\left(\zeta^{p_{i}}\right) \neq 0$, where $p_{i}$ denotes the projection of $p$ on $\mathbb{R}^{2} \times\left(S^{1}\right)^{i+1}$, the product of $\mathbb{R}^{2}$ and the first $i+1$ copies of $S^{1}$, that is $\zeta^{p_{i}}=\left(\xi_{1}^{p}, \xi_{2}^{p}, \theta_{0}^{p}, \ldots, \theta_{i}^{p}\right)$.

Proposition 3.2. For $n \geq 0$, the local diffeomorphism $\phi^{n}$ and the smooth functions $\nu_{n}, \eta_{n}$, and $\mu_{n}$ satisfy (15), and thus convert locally the n-trailer system into a Kumpera-Ruiz normal form.

Proof of Proposition 3.2. We will prove that the relation (15) holds for $n \geq 0$ by induction on the number $n$ of trailers. Relation (15) is clearly true for $n=0$. Assume that it holds for $n-1$ trailers, that is

$$
\begin{aligned}
& \phi_{*}^{n-1}\left(\tau_{1}^{n-1}\right)=\left(\nu_{n-1} \circ \psi^{n-1}\right) \kappa_{1}^{n+2} \\
& \phi_{*}^{n-1}\left(\tau_{2}^{n-1}\right)=\left(\eta_{n-1} \circ \psi^{n-1}\right) \kappa_{1}^{n+2}+\left(\mu_{n-1} \circ \psi^{n-1}\right) \kappa_{2}^{n+2} .
\end{aligned}
$$

The inductive definition of the $n$-trailer gives

$$
\begin{aligned}
\tau_{1}^{n} & =\frac{\partial}{\partial \theta_{n}} \\
\tau_{2}^{n} & =\sin \left(\theta_{n}-\theta_{n-1}\right) \tau_{1}^{n-1}+\cos \left(\theta_{n}-\theta_{n-1}\right) \tau_{2}^{n-1}
\end{aligned}
$$

Recall (see the proof of Th. 2.3, given in Appendix A) that for a diffeomorphism $\phi^{n}=\left(\phi^{n-1}, \phi_{n+3}\right)^{T}$ of $\mathbb{R}^{n+3}$, such that $\phi^{n-1}$ depends on the first $n+2$ coordinates only, and for a vector field $f$ on $\mathbb{R}^{n+3}$ of the form $f=\alpha f^{n-1}+f_{n+3}$, where $\alpha$ is a smooth function on $\mathbb{R}^{n+3}$, the vector field $f^{n-1}$ is the lift of a vector field on $\mathbb{R}^{n+2}$ (see Notation 2.1), and the only non-zero component of $f_{n+3}$ is the last one, we have

$$
\phi_{*}^{n}(f)=\left(\alpha \circ \psi^{n}\right) \phi_{*}^{n-1}\left(f^{n-1}\right)+\left(\left(\mathrm{L}_{f} \phi_{n+3}\right) \circ \psi^{n}\right) \frac{\partial}{\partial x_{n+3}} .
$$

Note that the vector field $\phi_{*}^{n-1}\left(f^{n-1}\right)$ is lifted along the $x_{n+3}$-coordinate, which is defined by $\phi_{n+3}$.

In the regular case, that is if $\theta_{i}^{p}-\theta_{i-1}^{p} \neq \pm \pi / 2 \bmod 2 \pi$, we take a regular prolongation and, using relations (16) and (18), we obtain:

$$
\begin{aligned}
& \phi_{*}^{n}\left(\tau_{2}^{n}\right)=\left(s_{n} \circ \psi^{n}\right) \phi_{*}^{n-1}\left(\tau_{1}^{n-1}\right)+\left(c_{n} \circ \psi^{n}\right) \phi_{*}^{n-1}\left(\tau_{2}^{n-1}\right)+\left(\left(\mathrm{L}_{\tau_{2}^{n}} \phi_{n+3}\right) \circ \psi^{n}\right) \frac{\partial}{\partial x_{n+3}} \\
& =\left(\left(s_{n} \nu_{n-1}+c_{n} \eta_{n-1}\right) \circ \psi^{n}\right) \kappa_{1}^{n+2}+\left(\left(c_{n} \mu_{n-1}\right) \circ \psi^{n}\right) \kappa_{2}^{n+2}+\left(\eta_{n} \circ \psi^{n}\right) \kappa_{1}^{n+3} \\
& =\left(c_{n} \mu_{n-1} \circ \psi^{n}\right)\left(\left(\frac{s_{n} \nu_{n-1}+c_{n} \eta_{n-1}}{c_{n} \mu_{n-1}} \circ \psi^{n}\right) \kappa_{1}^{n+2}+\kappa_{2}^{n+2}\right)+\left(\eta_{n} \circ \psi^{n}\right) \kappa_{1}^{n+3} \\
& =\left(\mu_{n} \circ \psi^{n}\right)\left(x_{n+3} \kappa_{1}^{n+2}+\kappa_{2}^{n+2}\right)+\left(\eta_{n} \circ \psi^{n}\right) \kappa_{1}^{n+3} \\
& =\left(\eta_{n} \circ \psi^{n}\right) \kappa_{1}^{n+3}+\left(\mu_{n} \circ \psi^{n}\right) \kappa_{2}^{n+3} \text {. }
\end{aligned}
$$

In the singular case, that is if $\theta_{i}^{p}-\theta_{i-1}^{p}= \pm \pi / 2 \bmod 2 \pi$, we take the singular prolongation and, using relations (17) and (18), we obtain:

$$
\begin{aligned}
\phi_{*}^{n}\left(\tau_{2}^{n}\right) & =\left(\left(s_{n} \nu_{n-1}+c_{n} \eta_{n-1}\right) \circ \psi^{n}\right)\left(\kappa_{1}^{n+2}+\left(\frac{c_{n} \mu_{n-1}}{s_{n} \nu_{n-1}+c_{n} \eta_{n-1}} \circ \psi^{n}\right) \kappa_{2}^{n+2}\right)+\left(\eta_{n} \circ \psi^{n}\right) \kappa_{1}^{n+3} \\
& =\left(\mu_{n} \circ \psi^{n}\right)\left(\kappa_{1}^{n+2}+x_{n+3} \kappa_{2}^{n+2}\right)+\left(\eta_{n} \circ \psi^{n}\right) \kappa_{1}^{n+3}=\left(\eta_{n} \circ \psi^{n}\right) \kappa_{1}^{n+3}+\left(\mu_{n} \circ \psi^{n}\right) \kappa_{2}^{n+3} .
\end{aligned}
$$


Moreover, in both cases, we have:

$$
\phi_{*}^{n}\left(\tau_{1}^{n}\right)=\left(\left(\mathrm{L}_{\tau_{1}^{n}} \phi_{n+3}\right) \circ \psi^{n}\right) \frac{\partial}{\partial x_{n+3}}=\left(\nu_{n} \circ \psi^{n}\right) \kappa_{1}^{n+3} .
$$

It follows that relation (15) holds for any $n \geq 0$.

\subsection{Conversion of an arbitrary Goursat structure into the $N$-trailer system}

Reversing the construction given in the proof of Proposition 3.2 leads to the following surprising result (already announced in [56] and proved in [57]), which states that the $n$-trailer system is a universal model for all Goursat structures (after the paper had been submitted, we learnt that an alternative proof of this result has been proposed by Montgomery and Zhitomirskiı [46]). This theorem will play a fundamental role in this article. Indeed, it will allow us to generalize local results known for the $n$-trailer, like the formula for the growth vector obtained by Jean [29], to all Goursat structures.

Theorem 3.3. Any Goursat structure on a manifold $M$ of dimension $n+3$ is locally equivalent, at any point $q$ in $M$, to the $n$-trailer considered around a suitably chosen point $p$ of its configuration space $\mathbb{R}^{2} \times\left(S^{1}\right)^{n+1}$.

Proof of Theorem 3.3. By Theorem 2.3, our Goursat structure is, in a small enough neighborhood of any point $q$ in $M$, equivalent to a Kumpera-Ruiz normal form $\kappa^{n+3}$. Denote by $y=\left(y_{1}, \ldots, y_{n+3}\right)$ the coordinates of $\kappa^{n+3}$ and put $\left(y_{1}^{q}, \ldots, y_{n+3}^{q}\right)=y(q)$.

Recall that, by definition, the pair of vector fields $\kappa^{n+3}$ is given by a sequence of prolongations $\kappa^{i}=\sigma_{i-3}$ $\circ \cdots \circ \sigma_{1}\left(\kappa^{3}\right)$, where $\sigma_{j}$ belongs to $\left\{R_{c_{j}}, S\right\}$, for $1 \leq j \leq i-3$ and $3 \leq i \leq n+3$. We call a coordinate $y_{i}$ such that $\kappa^{i}=S\left(\kappa^{i-1}\right)$ a singular coordinate, and a coordinate $y_{i}$ such that $\kappa^{i}=R_{c}\left(\kappa^{i-1}\right)$ a regular coordinate. It follows from the proof of Theorem 2.3 (see Appendix A) that for all singular coordinates we have $y_{i}^{q}=0$; but for regular coordinates, the constants $y_{i}^{q}$ can be arbitrary real numbers.

To prove Theorem 3.3 , we will define a point $p$ of $\mathbb{R}^{2} \times\left(S^{1}\right)^{n+1}$ whose coordinates

$$
\zeta(p)=\zeta^{p}=\left(\xi_{1}^{p}, \xi_{2}^{p}, \theta_{0}^{p}, \ldots, \theta_{n}^{p}\right)
$$

satisfy $(x \circ \zeta)(p)=y(q)$, where $x$ and $\zeta$ denote the coordinates used in the proof of Proposition 3.2. First, put the axle of the last trailer at $\left(y_{1}^{q}, y_{2}^{q}\right)$, that is $\left(\xi_{1}^{p}, \xi_{2}^{p}\right)=\left(y_{1}^{q}, y_{2}^{q}\right)$, and take $\theta_{0}^{p}=\arctan \left(y_{3}^{q}\right)$. Compute $x_{3}=\tan \left(\theta_{0}\right)$, $\mu_{0}=\cos \left(\theta_{0}\right), \nu_{0}=\sec ^{2}\left(\theta_{0}\right)$, and $\eta_{0}=0$. Then, take for $i=1$ up to $n$, the following values for the angles $\theta_{i}^{p} \bmod 2 \pi$. If the coordinate $y_{i+3}$ is singular then put $\theta_{i}^{p}=\theta_{i-1}^{p}+\pi / 2$ and compute the coordinate $x_{i+3}$ and the smooth functions $\mu_{i}, \nu_{i}$, and $\eta_{i}$ using (17). If $y_{i+3}$ is regular then put

$$
\theta_{i}^{p}=\arctan \left(\frac{\mu_{i-1}\left(\zeta^{p_{i}}\right) y_{i+3}^{q}-\eta_{i-1}\left(\zeta^{p_{i}}\right)}{\nu_{i-1}\left(\zeta^{p_{i}}\right)}\right)+\theta_{i-1}^{p}
$$

and compute the coordinate $x_{i+3}$ and the smooth functions $\mu_{i}, \nu_{i}$, and $\eta_{i}$ using (16). The result of this construction is that $(x \circ \zeta)(p)=y(q)$. By Proposition 3.2, the coordinates $x \circ \zeta$ convert the $n$-trailer into a Kumpera-Ruiz normal form. By the above defined construction, this normal form has the same singular coordinates as $\kappa^{n+3}$ and is defined around the same point of $\mathbb{R}^{n+3}$ (if we translate the regular coordinates in order to center them then those two Kumpera-Ruiz normal forms have the same constants in the regular prolongations). Hence, the diffeomorphism $\zeta^{-1} \circ x^{-1} \circ y$ gives the claimed equivalence.

\section{Singularity TyPE}

One of the main contributions of our paper is to introduce a new local invariant of Goursat structures. This invariant, which we call the singularity type, is defined in Section 4.3 using the notion of characteristic distributions, recalled in Section 4.1, and that of Jacquard's language, defined in Section 4.2. The concept 
of singularity type is illustrated and explained in the three consecutive subsections: for Goursat structures in small dimensions in Section 4.4, for Kumpera-Ruiz normal forms in Section 4.5, and for the $n$-trailer system in Section 4.6.

\subsection{Characteristic distributions}

A characteristic vector field of a distribution $\mathcal{D}$ is a vector field $f$ that belongs to $\mathcal{D}$ and satisfies $[f, \mathcal{D}] \subset \mathcal{D}$. The characteristic distribution of a distribution $\mathcal{D}$ is the module, over the ring of smooth functions, generated by all its characteristic vector fields. A characteristic distribution need not be of constant rank; but it follows directly from the Jacobi identity that a characteristic distribution is always involutive. The main ingredient in the definition of the singularity type will be the characteristic distributions $\mathcal{C}_{i}$ defined by the following result, which is apparently due to Cartan [11], although he did not state it explicitly in his published works. Its proof can be found in [32] and [43] (see also [8,46], and Appendix C), were slightly stronger versions are proved using the dual language of Pfaffian systems.

Proposition 4.1 (Cartan). Consider a Goursat structure $\mathcal{D}$ defined on a manifold of dimension $n \geq 4$. Each distribution $\mathcal{D}^{(i)}$, for $0 \leq i \leq n-4$, contains a unique involutive subdistribution $\mathcal{C}_{i} \subset \mathcal{D}^{(i)}$ that is characteristic for $\mathcal{D}^{(i+1)}$ and has constant corank one in $\mathcal{D}^{(i)}$.

\subsection{Jacquard's language}

An alphabet is a finite set $A$ whose elements are called letters. A word over $A$ is a finite sequence $w=$ $\left(w_{1}, \ldots, w_{l}\right)$, where each $w_{i}$ belongs to $A$ for $1 \leq i \leq l$. The integer $l$ is called the length of the word $w$ and we denote it by $|w|$. The empty word is the only word of length 0 . We denote it by $\epsilon$. By abuse of notation, we will often write $w_{1} \cdots w_{l}$ instead of $\left(w_{1}, \ldots, w_{l}\right)$ and $a^{l}$ instead of $(a, \ldots, a)$, for any letter $a$ repeated $l$ times. Now, call $A^{*}$ the set of all words, including the empty word, over the alphabet $A$. A language over $A$ is a subset $L$ of $A^{*}$. The concatenation of two words $v$ and $w$ over $A$ is the word $v w=\left(v_{1}, \ldots, v_{l}, w_{1}, \ldots, w_{m}\right)$, where $l=|v|$ and $m=|w|$. The concatenation of a language $L$ and a word $w$ over $A$ is the language

$$
L w=\{u w: u \in L\}
$$

The shift of a word $w=\left(w_{1}, \ldots, w_{l}\right)$ is the word $(w)^{\prime}=\left(w_{1}, \ldots, w_{l-1}\right)$. By definition, we take $(\epsilon)^{\prime}=\epsilon$.

We define now a sequence of languages that will play an important role in this paper, since they will describe all possible singularity types of a Goursat structure. For a fixed $n \geq 0$, consider the alphabet $A_{n}=\left\{a_{0}, \ldots, a_{n-1}\right\}$ (note that $A_{0}=\emptyset$ ). Following [24] define, inductively, the Jacquard language $J_{n}$ by $J_{0}=\{\epsilon\}, J_{1}=\left\{a_{0}\right\}$, and

$$
J_{n}=J_{n-1} a_{0} \cup J_{n-1} a_{1} \cup J_{n-2} a_{1} a_{2} \cup \ldots \cup J_{1} a_{1} a_{2} \cdots a_{n-1},
$$

for any integer $n \geq 2$. Clearly, the elements of $J_{n}$ are words over $A_{n}$ that all have length $n$. For example, we have $J_{2}=\left\{a_{0} a_{0}, a_{0} a_{1}\right\}$ and

$$
J_{3}=\left\{a_{0} a_{0} a_{0}, a_{0} a_{0} a_{1}, a_{0} a_{1} a_{0}, a_{0} a_{1} a_{1}, a_{0} a_{1} a_{2}\right\}
$$

It is easy to check, using an induction argument, that

$$
\operatorname{card}\left(J_{n}\right)=3 \operatorname{card}\left(J_{n-1}\right)-\operatorname{card}\left(J_{n-2}\right),
$$

for $n \geq 2$ (see [24]). 


\subsection{Definition of the singularity type}

We start with the definition of a sequence of canonical submanifolds, which will lead to the definition of the singularity type. Let $\mathcal{D}$ be a Goursat structure on a manifold $M$ of dimension $n$. For $0 \leq i \leq n-5$, define the subset $S_{0}^{(i)} \subset M$ by

$$
S_{0}^{(i)}=\left\{p \in M: \mathcal{D}^{(i)}(p)=\mathcal{C}_{i+1}(p)\right\}
$$

where the distribution $\mathcal{C}_{i}$ denotes the characteristic distribution of $\mathcal{D}^{(i+1)}$ (see Prop. 4.1). For $i \geq n-4$ define $S_{0}^{(i)}=\emptyset$.

Furthermore, starting from $S_{0}^{(i)}$ define, for $1 \leq j \leq i$, a sequence of subsets $M \supset S_{0}^{(i)} \supset \cdots \supset S_{j-1}^{(i)} \supset S_{j}^{(i)}$ $\supset \cdots \supset S_{i}^{(i)}$ by

$$
S_{j}^{(i)}=\left\{p \in S_{j-1}^{(i)}: \mathcal{D}^{(i-j)}(p) \cap T_{p} S_{j-1}^{(i)} \neq \mathcal{C}_{i-j}(p)\right\}
$$

For $j \geq i+1$ define $S_{j}^{(i)}=\emptyset$. The above definition is correct since, for any non-negative integers $i$ and $j$, the subset $S_{j}^{(i)} \subset M$ is a smooth submanifold of $M$ (we consider an empty set as smooth). Indeed, we have the following result, which will be proved later, in Section 4.5.

Proposition 4.2. Let $\mathcal{D}$ be a Goursat structure on a manifold $M$.

(i) For any non-negative integers $i$ and $j$, the subset $S_{j}^{(i)} \subset M$ defined by the relations (19) and (20) is either empty or a smooth submanifold of $M$ that has codimension $j+1$ in $M$.

(ii) For any non-negative integers $i, j$ and $k$ such that $k \neq j$ we have the following relation: $S_{j}^{(i+j)} \cap S_{k}^{(i+k)}=\emptyset$.

The fact that a point $p$ belongs to some submanifolds $S_{j}^{(i)}$, for two given non-negative integers $i$ and $j$, is invariantly related to the Goursat structure at this point $p$. This information, however, is in general redundant. For example, if $p$ belongs to $S_{j}^{(i)}$ we know, by definition, that $p$ belongs also to $S_{j-k}^{(i)}$, for $0 \leq k \leq j$, and that, by Proposition 4.2 , it does not belong to $S_{j+k}^{(i+k)}$, for $k \neq 0$. In the following definition we propose a way to encode the essential part of this information in a word of the Jacquard language (see Cor. 4.8, at the end of Sect. 4.5).

Definition 4.3. Let $\mathcal{D}$ be a Goursat structure defined in a neighborhood of a fixed point $p$ in a manifold of dimension $n$. The singularity type of $\mathcal{D}$ at $p$ is the word

$$
\delta_{\mathcal{D}}(p)=w_{n-4} \cdots w_{0}
$$

defined as follows: for $0 \leq i \leq n-4$, we take $w_{i}=a_{j+1}$ if there exists some integer $j \geq 0$ such that $p$ belongs to $S_{j}^{(i+j)}$; we take $w_{i}=a_{0}$ otherwise.

The correctness of this definition is assured by Proposition 4.2, which states that if there exists an integer $j \geq 0$ such that $p$ belongs to $S_{j}^{(i+j)}$ then this integer is unique. For some readers this definition may seem rather abstract at a first glance; but it appears quickly, once computed for concrete Goursat structures, that the singularity type really encodes essential geometric information. In fact, we will see that the growth vector and the abnormal curves of a Goursat structure are given by its singularity type (see Sects. 5 and 6).

The singularity type should not be confused with the codes used in [12] and [50] to label Kumpera-Ruiz normal forms. Indeed, the singularity type is, by construction, an invariant of a given Goursat structure; while the codes of [12] and [50] are not invariant: a given Goursat structure can have different codes, depending on how it is represented by a Kumpera-Ruiz normal form. 


\subsection{Low dimensional examples}

For any Goursat structure on a manifold of dimension three or four the singularity type is equal, at any point, to $\epsilon$ or $a_{0}$, respectively. That is, the singularity type of a contact or an Engel structure does not depend on the point at which the distribution is considered. This should be compared with the singularity type of a Goursat structure on a five-manifold, which can be either $a_{0} a_{0}$ or $a_{0} a_{1}$ at a given point $p$, depending on whether or not the Goursat structure can be converted into Goursat normal form in a small enough neighborhood of $p$. Indeed, for the Goursat structure spanned by the regular Kumpera-Ruiz normal form (7), the canonical submanifold $S_{0}^{(0)}$ is empty, and thus the singularity type equals $a_{0} a_{0}$ at each point of $\mathbb{R}^{5}$; for the Goursat structure spanned by the singular Kumpera-Ruiz normal form (8) we have $S_{0}^{(0)}=\left\{x_{5}=0\right\}$, and thus the singularity type equals $a_{0} a_{1}$ if $x_{5}=0$; and $a_{0} a_{0}$ if $x_{5} \neq 0$.

Let us give one more example, in dimension six. Consider the distribution $\mathcal{D}$ spanned by the following Kumpera-Ruiz normal form on $\mathbb{R}^{6}$ :

$$
\left(\frac{\partial}{\partial x_{6}},\left(x_{6}+c_{6}\right) \frac{\partial}{\partial x_{5}}+\frac{\partial}{\partial x_{4}}+x_{5} x_{4} \frac{\partial}{\partial x_{3}}+x_{5} x_{3} \frac{\partial}{\partial x_{2}}+x_{5} \frac{\partial}{\partial x_{1}}\right)
$$

where the constant $c_{6}$ is either equal to 0 or 1 . For both values of $c_{6}$, we have $S_{0}^{(0)}=\emptyset$ and $S_{0}^{(1)}=\left\{x_{5}=0\right\}$. For $c_{6}=1$ the submanifold $S_{1}^{(1)}$ is empty (in a small enough neighborhood of zero); for $c_{6}=0$ we have $S_{1}^{(1)}=\left\{x_{5}=x_{6}=0\right\}$. Therefore, the singularity type of $\mathcal{D}$ at zero equals $a_{0} a_{1} a_{0}$ if $c_{6}=1$; and equals $a_{0} a_{1} a_{2}$ if $c_{6}=0$.

\subsection{Singularity type of Kumpera-Ruiz normal forms}

Let $\kappa^{n}$ be a Kumpera-Ruiz normal form on $\mathbb{R}^{n}$ obtained by the inductive procedure described in Section 2 . We define inductively the word $\delta_{\kappa^{n}}$ of $J_{n-3}$ by $\delta_{\kappa^{3}}=\epsilon, \delta_{\kappa^{4}}=a_{0}$, and, for $n \geq 5$, by the relations

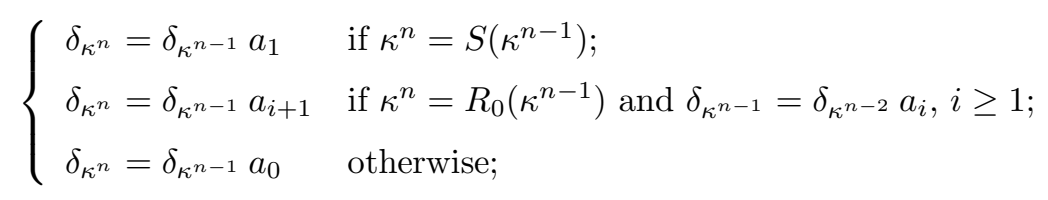

where the maps $S$ and $R_{c}$ denote respectively the singular and regular prolongations defined in Section 2. This definition leads to a characterization of the singularity type in the coordinates of the Kumpera-Ruiz normal form (see Cor. 4.6 below). We start with a proposition that will allow us to prove the results that were announced, without proof, in Section 4.3.

Proposition 4.4. Consider the distribution defined on $\mathbb{R}^{n}$ by a Kumpera-Ruiz normal form $\kappa^{n}$. For any pair of integers $i$ and $j$ such that $0 \leq j \leq i$ and for any pair of words $w_{1}$ and $w_{2}$ such that $w=w_{1} a_{1} a_{2} \cdots a_{j+1} w_{2}$ belongs to $J_{n-3}$ and $\left|w_{2}\right|=i-j$, we have $\delta_{\kappa^{n}}=w$ if and only if zero belongs to $S_{j}^{(i)}$. Moreover, if a submanifold $S_{j}^{(i)}$ contains zero then, in the coordinates $\left(x_{1}, \ldots, x_{n}\right)$ of the Kumpera-Ruiz normal form $\kappa^{n}$, we have

$$
S_{j}^{(i)}=\left\{x_{n-i}=0, \ldots, x_{n-i+j}=0\right\}
$$

where $0 \leq i \leq n-5$ and $0 \leq j \leq i$.

The proof of Proposition 4.4 will be based on the following lemma, which is a direct consequence of the definition of Kumpera-Ruiz normal forms given in Section 2 (see Def. 2.2); its proof is straightforward. Note that below all vector fields $\kappa_{2}^{n-i}$ should be considered as vector fields on $\mathbb{R}^{n}$, obtained from $\kappa_{2}^{n-i}$ by $i$ successive lifts, as defined by Notation 2.1 (see Sect. 2). 
Lemma 4.5. Let $\mathcal{D}$ be a Goursat structure on $\mathbb{R}^{n}$ spanned by a Kumpera-Ruiz normal form $\kappa^{n}=\left(\kappa_{1}^{n}, \kappa_{2}^{n}\right)$. Suppose that $\kappa^{n}=\sigma_{n-3} \circ \cdots \circ \sigma_{1}\left(\kappa^{3}\right)$ and denote $\kappa^{n-i}=\sigma_{n-3-i} \circ \cdots \circ \sigma_{1}\left(\kappa^{3}\right)$. The derived flag of $\mathcal{D}$ is given by

$$
\mathcal{D}^{(i)}=\left(\frac{\partial}{\partial x_{n}}, \ldots, \frac{\partial}{\partial x_{n-i}}, \kappa_{2}^{n-i}\right), \quad \text { for } \quad 0 \leq i \leq n-3 .
$$

The characteristic distribution $\mathcal{C}_{i} \subset \mathcal{D}^{(i)}$ of $\mathcal{D}^{(i+1)}$ is given by

$$
\mathcal{C}_{i}=\left(\frac{\partial}{\partial x_{n}}, \ldots, \frac{\partial}{\partial x_{n-i}}\right), \quad \text { for } \quad 0 \leq i \leq n-4
$$

Moreover, if $\delta_{\kappa^{n}}=w_{1} a_{1} a_{2} \cdots a_{j} a w_{2}$, where $\left|w_{2}\right|=i-j$ and $a \in\left\{a_{0}, a_{1}, a_{j+1}\right\}$, then we have

$$
\kappa_{2}^{n-i+l}=\sum_{k=1}^{l} x_{n-i+k} \frac{\partial}{\partial x_{n-i+k-1}}+\frac{\partial}{\partial x_{n-i-1}}+x_{n-i} \kappa_{2}^{n-i-1},
$$

for $0 \leq l \leq j-1$.

Proof of Proposition 4.4. For any fixed integer $i$ such that $0 \leq i \leq n-5$, we will prove the proposition by induction on the integer $j$, for $0 \leq j \leq i$. We start with $j=0$. Assume that $\delta_{\kappa^{n}}=w_{1} a w_{2}$, where $\left|w_{2}\right|=i$ and $a \in A_{n-1}$. If $a \neq a_{1}$ then the definition of $\delta_{\kappa^{n}}$ implies

$$
\kappa_{2}^{n-i}=\left(x_{n-i}+c_{n-i}\right) \frac{\partial}{\partial x_{n-i-1}}+\kappa_{2}^{n-i-1}
$$

and it follows from relation (23) that

$$
\mathcal{D}^{(i)}=\left(\frac{\partial}{\partial x_{n}}, \ldots, \frac{\partial}{\partial x_{n-i}},\left(x_{n-i}+c_{n-i}\right) \frac{\partial}{\partial x_{n-i-1}}+\kappa_{2}^{n-i-1}\right) .
$$

Together with relation (24) this expression implies that, for any point $p \in \mathbb{R}^{n}$, we have $\mathcal{D}^{(i)}(p) \neq \mathcal{C}_{i+1}(p)$, which implies that $S_{0}^{(i)}$ is empty. In particular $0 \notin S_{0}^{(i)}$. Otherwise $a=a_{1}$, and then the definition of $\delta_{\kappa^{n}}$ implies

$$
\kappa_{2}^{n-i}=\frac{\partial}{\partial x_{n-i-1}}+x_{n-i} \kappa_{2}^{n-i-1}
$$

and it follows, again from relation (23), that

$$
\mathcal{D}^{(i)}=\left(\frac{\partial}{\partial x_{n}}, \ldots, \frac{\partial}{\partial x_{n-i}}, \frac{\partial}{\partial x_{n-i-1}}+x_{n-i} \kappa_{2}^{n-i-1}\right) .
$$

Hence, for any point $p \in \mathbb{R}^{n}$, we have $\mathcal{D}^{(i)}(p)=\mathcal{C}_{i+1}(p)$ if and only if $x_{n-i}(p)=0$. In particular $0 \in S_{0}^{(i)}$. It follows that Proposition 4.4 is true when $j=0$.

Now, assume that Proposition 4.4 is true up to the integer $j-1$ and that

$$
\delta_{\kappa^{n}}=w_{1} a_{1} a_{2} \cdots a_{j} a w_{2},
$$

where $\left|w_{2}\right|=i-j$ and $a \in\left\{a_{1}, a_{0}, a_{j+1}\right\}$. Since

$$
S_{j-1}^{(i)}=\left\{x_{n-i}=\cdots=x_{n-i+j-1}=0\right\},
$$


we have

$$
T_{p} S_{j-1}^{(i)}=\left\{\frac{\partial}{\partial x_{n}}, \ldots, \frac{\partial}{\partial x_{n-i+j}}, \frac{\partial}{\partial x_{n-i-1}}, \ldots, \frac{\partial}{\partial x_{1}}\right\} .
$$

Observe that relation (23) gives

$$
\mathcal{D}^{(i-j)}=\left(\frac{\partial}{\partial x_{n}}, \ldots, \frac{\partial}{\partial x_{n-i+j}}, \kappa_{2}^{n-i+j}\right) .
$$

Moreover, it follows from relation (25), taken for $l=j-1$, that

$$
\kappa_{2}^{n-i+j-1}=\sum_{k=1}^{j-1} x_{n-i+k} \frac{\partial}{\partial x_{n-i+k-1}}+\frac{\partial}{\partial x_{n-i-1}}+x_{n-i} \kappa_{2}^{n-i-1} .
$$

If $a=a_{1}$ then the definition of $\delta_{\kappa^{n}}$ implies

$$
\kappa_{2}^{n-i+j}=\frac{\partial}{\partial x_{n-i+j-1}}+x_{n-i+j} \kappa_{2}^{n-i+j-1} .
$$

Therefore the vector field $\kappa_{2}^{n-i+j}$ that appears in the relation (26) is, using (27), given by

$$
\kappa_{2}^{n-i+j}=\frac{\partial}{\partial x_{n-i+j-1}}+x_{n-i+j}\left(\sum_{k=1}^{j-1} x_{n-i+k} \frac{\partial}{\partial x_{n-i+k-1}}+\frac{\partial}{\partial x_{n-i-1}}+x_{n-i} \kappa_{2}^{n-i-1}\right) .
$$

Hence, for any $p \in S_{j-1}^{(i)}$, we have $\mathcal{D}^{(i-j)}(p) \cap T_{p} S_{j-1}^{(i)}=\mathcal{C}_{i-j}(p)$, which implies that $S_{j}^{(i)}$ is empty. In particular $0 \notin S_{j}^{(i)}$. If $a=a_{0}$ then the definition of $\delta_{\kappa^{n}}$ implies

$$
\kappa_{2}^{n-i+j}=\left(x_{n-i+j}+c\right) \frac{\partial}{\partial x_{n-i+j-1}}+\kappa_{2}^{n-i+j-1},
$$

where $c \neq 0$. Therefore the vector field $\kappa_{2}^{n-i+j}$ that appears in the relation (26) is, using (27), given by

$$
\kappa_{2}^{n-i+j}=\left(x_{n-i+j}+c\right) \frac{\partial}{\partial x_{n-i+j-1}}+\sum_{k=1}^{j-1} x_{n-i+k} \frac{\partial}{\partial x_{n-i+k-1}}+\frac{\partial}{\partial x_{n-i-1}}+x_{n-i} \kappa_{2}^{n-i-1} .
$$

Thus for any $p \in S_{j-1}^{(i)}$ we have $\mathcal{D}^{(i-j)}(p) \cap T_{p} S_{j-1}^{(i)}=\mathcal{C}_{i-j}(p)$, which implies that $S_{j}^{(i)}$ is empty (at least in small enough neighborhood of zero). In particular $0 \notin S_{j}^{(i)}$. Finally, if we have $a=a_{j+1}$ then the definition of $\delta_{\kappa^{n}}$ implies

$$
\kappa_{2}^{n-i+j}=x_{n-i+j} \frac{\partial}{\partial x_{n-i+j-1}}+\kappa_{2}^{n-i+j-1} .
$$

Therefore the vector field $\kappa_{2}^{n-i+j}$ that appears in the relation (26) is, using (27), given by

$$
\kappa_{2}^{n-i+j}=\sum_{k=1}^{j} x_{n-i+k} \frac{\partial}{\partial x_{n-i+k-1}}+\frac{\partial}{\partial x_{n-i-1}}+x_{n-i} \kappa_{2}^{n-i-1} .
$$


Thus for any $p \in S_{j-1}^{(i)}$ we have $\mathcal{D}^{(i-j)}(p) \cap T_{p} S_{j-1}^{(i)} \neq \mathcal{C}_{i-j}(p)$ if and only if

$$
x_{n-i}(p)=\cdots=x_{n-i+j}(p)=0 .
$$

In particular, we have $0 \in S_{j}^{(i)}$, which ends the proof.

Proof of Proposition 4.2. Consider a Goursat structure $\mathcal{D}$ defined on a smooth manifold $M$ of dimension $n$.

First Item: Item (i) of Proposition 4.2 follows directly from Proposition 4.4, which states that, in the coordinates of a Kumpera-Ruiz normal form, the restriction of each set $S_{j}^{(i)}$ to a small enough neighborhood of zero is either empty or smooth. Indeed, by Theorem 2.3 , the Goursat structure $\mathcal{D}$ is locally equivalent, at any point $p$ in $M$, to a Kumpera-Ruiz normal form centered at $p$; and hence the restriction of each set $S_{j}^{(i)}$ to a small enough neighborhood of any point $p$ in $M$ is either empty or smooth. This obviously implies that the whole set $S_{j}^{(i)}$ is either empty or smooth.

Second Item: We will prove Item (ii) by contradiction. Let $\mathcal{D}$ be a Goursat structure such that at a point $p$ we have $p \in S_{j}^{(i+j)} \cap S_{k}^{(i+k)}$ for a given triple of non-negative integers $i, j$, and $k$, such that $k<j$. Take a Kumpera-Ruiz normal form $\kappa^{n}$, centered at $p$, that is locally equivalent to $\mathcal{D}$ at $p$. In the coordinates of $\kappa^{n}$, we have $0 \in S_{j}^{(i+j)} \cap S_{k}^{(i+k)}$. Let $w=\delta_{\kappa^{n}}$ be the word uniquely attached to $\kappa^{n}$ by $(21)$, and denote the letters of $w$ by $w=w_{n-4} \cdots w_{0}$. By Proposition 4.4, we have both $w_{i+l}=a_{j-l+1}$, for $0 \leq l \leq j$, and $w_{i+l}=a_{k-l+1}$, for $0 \leq l \leq k$. In particular, we have $w_{i}=a_{j+1}$ and $w_{i}=a_{k+1}$, which is impossible since the inequality $k<j$ implies that $a_{k+1} \neq a_{j+1}$.

The three Corollaries listed below follow directly from Proposition 4.4 and from the definition of the singularity type.

Corollary 4.6. Let $\mathcal{D}$ be the Goursat structure spanned on $\mathbb{R}^{n}$ by a Kumpera-Ruiz normal form $\kappa^{n}$. We have

$$
\delta_{\mathcal{D}}(0)=\delta_{\kappa^{n}},
$$

that is the singularity type at zero of $\kappa^{n}$ is given by $\delta_{\kappa^{n}}$.

Corollary 4.7. Let $\mathcal{D}$ be a Goursat structure defined in a neighborhood of a fixed point $p$ of a manifold of dimension $n$. For any integers such that $0 \leq j \leq i$, the point $p$ belongs to $S_{j}^{(i)}$ if and only if the singularity type of $\mathcal{D}$ at $p$ is of the form $\delta_{\mathcal{D}}(p)=w_{1} a_{1} a_{2} \cdots a_{j+1} w_{2}$, with $\left|w_{2}\right|=i-j$.

Corollary 4.8. The singularity type of any Goursat structure on a manifold of dimension $n$ belongs to the Jacquard language $J_{n-3}$. Conversely, any word of $J_{n-3}$ is the singularity type of some Goursat structure.

\subsection{Singularity type of the $N$-trailer system}

In this section, we come back to the $n$-trailer system, for which we compute the singularity type. Our study stays very close to that of Jean [29]. For the $n$-trailer system $\tau^{n}$ at a configuration $p=\left(\xi_{1}, \xi_{2}, \theta_{0}, \ldots, \theta_{n}\right)$ of $\mathbb{R}^{2} \times\left(S^{1}\right)^{n+1}$, we define inductively, following [29], a word $\delta_{\tau^{n}}(p)=w_{1} \cdots w_{n}$ of $J_{n}$ by $w_{1}=a_{0}$ and, for $i=2, \ldots, n$, by the relations

$$
\begin{cases}w_{i}=a_{1} & \text { if } \theta_{i}-\theta_{i-1}= \pm \frac{\pi}{2} \\ w_{i}=a_{k+1} & \text { if } w_{i-1}=a_{k}, \text { for } k \geq 1, \text { and } \tan \left(\theta_{i}-\theta_{i-1}\right)=\sin \left(\theta_{i-1}-\theta_{i-2}\right) \\ w_{i}=a_{0} & \text { otherwise. }\end{cases}
$$

This definition leads to a characterization of the singularity type in the coordinates of the $n$-trailer system, which coincides with the stratification of the singular locus given in [29]. 
Proposition 4.9. Let $\mathcal{D}$ be the Goursat structure spanned by the $n$-trailer system $\tau^{n}$ on $\mathbb{R}^{2} \times\left(S^{1}\right)^{n+1}$. For any point $p$ of $\mathbb{R}^{2} \times\left(S^{1}\right)^{n+1}$, we have

$$
\delta_{\mathcal{D}}(p)=\delta_{\tau^{n}}(p) .
$$

Moreover, in the coordinates $\left(\xi_{1}, \xi_{2}, \theta_{0}, \ldots, \theta_{n}\right)$ of the $n$-trailer system, we have

$$
\begin{aligned}
S_{j}^{(i)}= & \left\{p \in \mathbb{R}^{2} \times\left(S^{1}\right)^{n+1}: \theta_{n-i}-\theta_{n-i-1}= \pm \frac{\pi}{2} \text { and } \tan \left(\theta_{n-i+k}-\theta_{n-i+k-1}\right)=\sin \left(\theta_{n-i+k-1}-\theta_{n-i+k-2}\right),\right. \\
& \quad \text { for } 1 \leq k \leq j\}
\end{aligned}
$$

for $0 \leq i \leq n-2$ and $0 \leq j \leq i$.

Like in Section 3 , for a fixed point $p=\left(\xi_{1}, \xi_{2}, \theta_{0}, \ldots, \theta_{n}\right)$ of $\mathbb{R}^{2} \times\left(S^{1}\right)^{n+1}$, we will use the notation $s_{i}=$ $\sin \left(\theta_{i}-\theta_{i-1}\right)$ and $c_{i}=\cos \left(\theta_{i}-\theta_{i-1}\right)$. Moreover, we define the product $\pi_{n i}^{l k}$ by the relation $\pi_{n i}^{l k}=\prod_{j=k}^{l} c_{n-i+j}$, if $0 \leq k \leq l$, and by $\pi_{n i}^{l k}=1$, if $k>l$.

The proof of Proposition 4.9 will use the two following lemmas. The first one is analogous to Lemma 4.5, of Section 4.5 , it shows that the characteristic distributions of the $n$-trailer are rectified in $\left(\xi_{1}, \xi_{2}, \theta_{0}, \ldots, \theta_{n}\right)$ coordinates. Its proof is straightforward and left to the reader.

Lemma 4.10. Let $\mathcal{D}$ be the distribution spanned by the $n$-trailer system $\left(\tau_{1}^{n}, \tau_{2}^{n}\right)$. The derived flag of $\mathcal{D}$ is given by

$$
\mathcal{D}^{(i)}=\left(\frac{\partial}{\partial \theta_{n}}, \ldots, \frac{\partial}{\partial \theta_{n-i}}, s_{n-i} \frac{\partial}{\partial \theta_{n-i-1}}+c_{n-i} \tau_{2}^{n-i-1}\right), \quad \text { for } 0 \leq i \leq n
$$

The characteristic distributions $\mathcal{C}_{i}$ of $\mathcal{D}^{(i+1)}$ are given by

$$
\mathcal{C}_{i}=\left(\frac{\partial}{\partial \theta_{n}}, \ldots, \frac{\partial}{\partial \theta_{n-i}}\right), \quad \text { for } 0 \leq i \leq n-1 .
$$

Moreover, we have

$$
\mathcal{D}^{(i-j)}=\left(\frac{\partial}{\partial \theta_{n}}, \ldots, \frac{\partial}{\partial \theta_{n-i+j}}, \sum_{k=1}^{j+1}\left(s_{n-i+k-1}\right)\left(\pi_{n i}^{j k}\right) \frac{\partial}{\partial \theta_{n-i+k-2}}+\pi_{n i}^{j 0} \tau_{2}^{n-i-1}\right)
$$

for $0 \leq i \leq n-1$ and $0 \leq j \leq i$.

The lemma below is essentially a trigonometric identity and its proof, based on an induction argument, is also straightforward. We also leave it to the reader.

Lemma 4.11. Let $\left(\xi_{1}, \xi_{2}, \theta_{0}, \ldots, \theta_{n}\right) \in \mathbb{R}^{2} \times\left(S^{1}\right)^{n+1}$ be a fixed point of the configuration space of the $n$-trailer. If $\theta_{n-i}-\theta_{n-i-1}= \pm \frac{\pi}{2}$ and $\tan \left(\theta_{n-i+k}-\theta_{n-i+k-1}\right)=\sin \left(\theta_{n-i+k-1}-\theta_{n-i+k-2}\right)$, for $1 \leq k \leq j-1$, then

$$
\sum_{k=1}^{j}\left(s_{n-i+k-1}\right)\left(\pi_{n i}^{j k}\right) \frac{\partial}{\partial \theta_{n-i+k-2}}=s_{n-i+j-1} c_{n-i+j}\left(\sum_{k=1}^{j} \frac{\partial}{\partial \theta_{n-i+k-2}}\right) .
$$

Proof of Proposition 4.9. To start with, let us prove relation (29). For any fixed $i$ we will prove the result by induction on $j$. The relations (30) and (31) imply that $\mathcal{D}^{(i)}(p)=\mathcal{C}_{i+1}(p)$ if and only if $c_{n-i}=0$. That is, if and 
only if $\theta_{n-i}-\theta_{n-i-1}= \pm \frac{\pi}{2}$, which implies that the proposition is true for $j=0$. Now, assume the proposition true up to $j-1$. Relation (32) implies that $\mathcal{D}^{(i-j)}$ is given by

$$
\left(\frac{\partial}{\partial \theta_{n}}, \ldots, \frac{\partial}{\partial \theta_{n-i+j}}, \sum_{k=1}^{j+1}\left(s_{n-i+k-1}\right)\left(\pi_{n i}^{j k}\right) \frac{\partial}{\partial \theta_{n-i+k-2}}+\pi_{n i}^{j 0} \tau_{2}^{n-i-1}\right) .
$$

The induction assumption, together with Lemma 4.11, implies that for any point $p$ that belongs to $S_{j-1}^{(i)}$ the linear subspace $\mathcal{D}^{(i-j)}(p)$ is equal to

$$
\left(\frac{\partial}{\partial \theta_{n}}, \ldots, \frac{\partial}{\partial \theta_{n-i+j}}, s_{n-i+j} \frac{\partial}{\partial \theta_{n-i+j-1}}+\left(s_{n-i+j-1} c_{n-i+j}\right)\left(\sum_{k=1}^{j} \frac{\partial}{\partial \theta_{n-i+k-2}}\right)\right) .
$$

The induction assumption says that

$$
S_{j-1}^{(i)}=\left\{p: \theta_{n-i}-\theta_{n-i-1}= \pm \frac{\pi}{2} \text { and } \tan \left(\theta_{n-i+k}-\theta_{n-i+k-1}\right)=\sin \left(\theta_{n-i+k-1}-\theta_{n-i+k-2}\right), \text { for } 1 \leq k \leq j-1\right\} .
$$

Since

$$
T_{p} S_{j-1}^{(i)}=\left(\frac{\partial}{\partial \theta_{n}}, \ldots, \frac{\partial}{\partial \theta_{n-i+j}}, \sum_{k=1}^{j+1} \frac{\partial}{\partial \theta_{n-i+k-2}}, \frac{\partial}{\partial \theta_{n-i-2}}, \ldots, \frac{\partial}{\partial \theta_{0}}, \frac{\partial}{\partial \xi_{2}}, \frac{\partial}{\partial \xi_{1}}\right),
$$

we have $\mathcal{D}^{(i-j)}(p) \cap T_{p} S_{j-1}^{(i)} \neq \mathcal{C}_{i-j}(p)$ if and only if $s_{n-i+j}=s_{n-i+j-1} c_{n-i+j}$. That is, we have $p \in S_{j}^{(i)}$ if and only if $p \in S_{j-1}^{(i)}$ and $\tan \left(\theta_{n-i+j}-\theta_{n-i+j-1}\right)=\sin \left(\theta_{n-i+j-1}-\theta_{n-i+j-2}\right)$, which ends the induction argument.

Now, the form of $S_{j}^{(i)}$ obtained in the previous paragraph together with the definitions of $\delta_{\mathcal{D}}$ and $\delta_{\tau^{n}}$, imply directly that $\delta_{\mathcal{D}}(p)=\delta_{\tau^{n}}(p)$.

\section{Growth veCtor}

Perhaps the most elementary invariant of distributions is the growth vector. The aim of this section is to study relations between two invariants: the growth vector and the singularity type. We recall the definition of the growth vector in Section 5.1, where we also give a very elegant characterization, due to Murray, of Goursat structures equivalent to Goursat normal form. In Section 5.2 we provide another important result, due to Jean, which gives the growth vector of the $n$-trailer system. Next we will show that the growth vector and the singularity type are equivalent as local invariants: in Section 5.3 we show that the growth vector is a function of the singularity type while in Section 5.4 we prove the converse, namely, that the growth vector determines the singularity type. The definition of the singularity type given in Section 5.1 is geometric and independent of a particular representation of the Goursat structure. We can, however, hardly use it to calculate this new invariant. For this reason, we give in Section 5.5 a formula to calculate the singularity type in terms of the growth vector.

\subsection{Lie flag and growth vector}

The Lie flag of a distribution $\mathcal{D}$ is the sequence of modules of vector fields $\mathcal{D}_{0} \subset \mathcal{D}_{1} \subset \cdots$ defined inductively by

$$
\mathcal{D}_{0}=\mathcal{D} \quad \text { and } \quad \mathcal{D}_{i+1}=\mathcal{D}_{i}+\left[\mathcal{D}_{0}, \mathcal{D}_{i}\right], \quad \text { for } \quad i \geq 0 .
$$

This sequence should not be confused with the derived flag (1), introduced at the beginning of the article. In general these two sequences are different. Nevertheless, for any point $p$ in the underlying manifold $M$, the 
inclusion $\mathcal{D}_{i}(p) \subset \mathcal{D}^{(i)}(p)$ holds, for $i \geq 0$. Note that for a Goursat structure, unlike the elements of the derived flag, the elements of the Lie flag are not necessarily distributions of constant rank.

A distribution $\mathcal{D}$ is completely nonholonomic if, for each point $p$ in $M$, there exists an integer $N(p)$ such that $\mathcal{D}_{N(p)}(p)=T_{p} M$. The smallest such integer is called the nonholonomy degree of $\mathcal{D}$ at $p$ and we denote it by $N_{p}$. For a Goursat structure on a manifold of dimension $n$, the inequality $N_{p} \leq 2^{n-3}$ holds for each point $p$ in $M$ (see e.g. [35]). For the $n$-trailer system, sharper bounds were obtained in [29,42,63]. It follows from our Theorem 3.3, which states that any Goursat structure is locally equivalent to the $n$-trailer system, that they hold also for any Goursat structure.

Definition 5.1. Let $\mathcal{D}$ be a completely nonholonomic distribution. Put $d_{i}(p)=\operatorname{dim} \mathcal{D}_{i}(p)$, for $0 \leq i \leq N_{p}$. The growth vector at $p$ of the distribution $\mathcal{D}$ is the finite sequence $\left(d_{0}(p), \ldots, d_{N_{p}}(p)\right)$.

Recall that if at a given point a Goursat structure can be converted into Goursat normal form (3) then this point is called regular and that otherwise it is called singular (see Sect. 2). The set of singular points is called the singular locus. An elegant characterization of this set, that emphasizes the importance of the growth vector in the study of Goursat structures, has been obtained by Murray [53]. A different characterization can be found in [32] and [39].

Theorem 5.2 (Murray). Let $p$ be a point in a manifold $M$ of dimension $n$. A Goursat structure on $M$ can be converted into Goursat normal form in a small enough neighborhood of $p$ if and only if $\mathcal{D}_{i}(p)=\mathcal{D}^{(i)}(p)$, for $0 \leq i \leq n-2$.

\subsection{Growth vector of the $N$-trailer system}

Let $d=\left(d_{0}, \ldots, d_{N}\right)$ be a finite sequence of integers such that $d_{0}=2, d_{N}=n$, and $d_{i} \leq d_{i+1} \leq d_{i}+1$, for $0 \leq i \leq N-1$. The dual of the sequence $d$ is the sequence $d^{*}=\left(d_{2}^{*}, \ldots, d_{n}^{*}\right)$ defined by

$$
d_{i}^{*}=\operatorname{card}\left\{j \geq 0: d_{j}<i\right\}+1 \quad \text { for } \quad 2 \leq i \leq n .
$$

In other words, the integer $d_{i}^{*}$ indicates the first position, starting from the left, where the integer $i$ appears in $d$. We obviously have $d_{2}^{*}=1$ and $d_{n}^{*}=N+1$. It is trivial to check that each sequence $d$ is uniquely defined by its dual $d^{*}$. For example, we have the following dual sequences: $(2,3,4,5,6)^{*}=(1,2,3,4,5)$, $(2,3,4,5,5,5,6)^{*}=(1,2,3,4,7)$, and $(2,3,4,4,5,5,5,6)^{*}=(1,2,3,5,8)$.

Now, following [29], we define a set of functions that will allow us to obtain a formula that gives the growth vector of an arbitrary Goursat structure at an arbitrary point, as a function of its singularity type at this point. We start with Jean's formula [29] for the $n$-trailer. Recall that $J_{n}$ denotes the Jacquard language (see Sect. 4) and that the shift of a word is defined by $\left(w_{1} \cdots w_{n}\right)^{\prime}=w_{1} \cdots w_{n-1}$ and $(\epsilon)^{\prime}=\epsilon$ (we will denote $\left(w^{\prime}\right)^{\prime}$ by $\left.w^{\prime \prime}\right)$.

For any $i \geq 2$, we define functions $\beta_{i}: \bigcup_{n \geq i-3} J_{n} \rightarrow \mathbb{N}$. We take $\beta_{2}(w)=1, \beta_{3}(w)=2$, and $\beta_{4}(w)=3$, for any word $w$ in $\bigcup_{n \geq i-3} J_{n}$. If $i \geq 5$ then we define inductively, for any word $w$ in $\bigcup_{n \geq i-3} J_{n}$,

$$
\begin{cases}\beta_{i}(w)=\beta_{i-1}\left(w^{\prime}\right)+\beta_{i-2}\left(w^{\prime \prime}\right) & \text { if } w=\left(w^{\prime}\right) a_{1} \\ \beta_{i}(w)=2 \beta_{i-1}\left(w^{\prime}\right)-\beta_{i-2}\left(w^{\prime \prime}\right) & \text { if } w=\left(w^{\prime}\right) a_{k} \text { and } k \geq 2 \\ \beta_{i}(w)=\beta_{i-1}\left(w^{\prime}\right)+1 & \text { if } w=\left(w^{\prime}\right) a_{0} .\end{cases}
$$

For example, for the word $a_{0} a_{1} a_{0}$, we have:

$$
\begin{aligned}
& \beta_{5}\left(a_{0} a_{1} a_{0}\right)=\beta_{4}\left(a_{0} a_{1}\right)+1=3+1=4 \\
& \beta_{6}\left(a_{0} a_{1} a_{0}\right)=\beta_{5}\left(a_{0} a_{1}\right)+1=\left(\beta_{4}\left(a_{0}\right)+\beta_{3}(\epsilon)\right)+1=(3+2)+1=6 .
\end{aligned}
$$


Another example is given for the word $a_{0} a_{1} a_{2}$, for which we have:

$$
\begin{aligned}
& \beta_{5}\left(a_{0} a_{1} a_{2}\right)=\beta_{4}\left(a_{0} a_{1}\right)+1=3+1=4 \\
& \beta_{6}\left(a_{0} a_{1} a_{2}\right)=2 \beta_{5}\left(a_{0} a_{1}\right)-\beta_{4}\left(a_{0}\right)=2\left(\beta_{4}\left(a_{0}\right)+\beta_{3}(\epsilon)\right)-3=7 .
\end{aligned}
$$

Having recalled the functions $\beta_{i}$ we are now able to recall the formula, obtained by Jean [29], that gives the growth vector of the $n$-trailer system.

Theorem 5.3 (Jean). Consider the $n$-trailer system at a given point $p$ of its configuration space $\mathbb{R}^{2} \times\left(S^{1}\right)^{n+1}$ at which it has singularity type $\delta_{\tau^{n}}(p)$. The sequence of integers $\left(d_{2}^{*}(p), \ldots, d_{n+3}^{*}(p)\right)$ dual to the growth vector of the $n$-trailer system at $p$ is given by $d_{i}^{*}(p)=\beta_{i}\left(\delta_{\tau^{n}}(p)\right)$.

\subsection{Growth vector of Goursat structures}

The following result is fundamental. It shows that the growth vector of any Goursat structure is a function of its singularity type.

Theorem 5.4. Let $\mathcal{D}$ be a Goursat structure on a manifold $M$ of dimension $n \geq 3$, defined in a neighborhood of a given point $p$ in $M$ that has singularity type $\delta_{\mathcal{D}}(p)$. The sequence of integers $\left(d_{2}^{*}(p), \ldots, d_{n}^{*}(p)\right)$ dual to its growth vector at $p$ is given by $d_{i}^{*}(p)=\beta_{i}\left(\delta_{\mathcal{D}}(p)\right)$.

Proof of Theorem 5.4. Let $\mathcal{D}$ be a Goursat structure on a manifold $M$ of dimension $n \geq 3$, defined in a neighborhood of a given point $p$ in $M$. By Theorem 3.3, the Goursat structure $\mathcal{D}$ is locally equivalent at $p$ to the $n$-trailer system, considered around a well chosen point $q$ of its configuration space. By Theorem 5.3, the sequence of integers $\left(d_{2}^{*}(q), \ldots, d_{n+3}^{*}(q)\right)$ dual to the growth vector of the $n$-trailer system at $q$ is given by $d_{i}^{*}(q)=\beta_{i}\left(\delta_{\tau^{n}}(q)\right)$. By Proposition 4.9, the singularity type of the $n$-trailer system at $q$ equals $\delta_{\tau^{n}}(q)$. Since the singularity type is invariant under diffeomorphisms, we have $\delta_{\mathcal{D}}(p)=\delta_{\tau^{n}}(q)$. Since the growth vector is invariant under diffeomorphisms, the sequence of integers $\left(d_{2}^{*}(p), \ldots, d_{n+3}^{*}(p)\right)$ dual to the growth vector of $\mathcal{D}$ at $p$ is given by $d_{i}^{*}(p)=\beta_{i}\left(\delta_{\tau^{n}}(q)\right)=\beta_{i}\left(\delta_{\mathcal{D}}(p)\right)$.

The latter result obviously implies the following one, which gives the formula for the growth vector of an arbitrary Kumpera-Ruiz normal form.

Corollary 5.5. Let $\kappa^{n}$ be a Kumpera-Ruiz normal form on $\mathbb{R}^{n}$, for $n \geq 3$. The sequence $\left(d_{2}^{*}, \ldots, d_{n}^{*}\right)$ dual to its growth vector at zero is given by $d_{i}^{*}=\beta_{i}\left(\delta_{\kappa^{n}}\right)$.

\subsection{Growth vector and singularity type}

We proved in the previous section (Th. 5.4) that the singularity type of any Goursat structure at a given point determines its growth vector at this point. Now, we will prove the converse of this fact.

Theorem 5.6. Two Goursat structures have the same growth vector at a given point if and only if they have the same singularity type at this point.

The proof of Theorem 5.6 will be based on two lemmas:

Lemma 5.7. Let $i$ and $k$ be two integers such that $i \geq 1$ and $0 \leq k \leq i-1$. For any word $w$ in $\bigcup_{n \geq 1} J_{n}$ we have the following relations:

(i) $\beta_{i+4}\left(w a_{1} a_{2} \cdots a_{i}\right)=2 i+3$;

(ii) $\beta_{i+4}\left(w a_{1} a_{2} \cdots a_{i-k} a_{0}^{k}\right)=2 i-k+3$;

(iii) $\beta_{i+4}\left(w c_{1} \cdots c_{i}\right)=i+3$;

where $c_{j}$, for $1 \leq j \leq i$, are any letters satisfying $c_{j} \neq a_{1}$.

Proof of Lemma 5.\%. First Item. Item (i) is true if $i=1$ because, for any word $w$ in $\bigcup_{n \geq 1} J_{n}$, we have

$$
\beta_{5}\left(w a_{1}\right)=\beta_{4}(w)+\beta_{3}\left(w^{\prime}\right)=3+2=2 \cdot 1+3 .
$$


It is also true if $i=2$ because, for any word $w$ in $\bigcup_{n \geq 1} J_{n}$, we have

$$
\beta_{6}\left(w a_{1} a_{2}\right)=2 \cdot \beta_{5}\left(w a_{1}\right)-\beta_{4}(w)=2 \cdot 5-3=2 \cdot 2+3
$$

Now proceed by induction on $i \geq 3$. Assume that Item (i) is true up to $i-1$. Then, for any word $w$ in $\bigcup_{n \geq 1} J_{n}$, we have

$$
\begin{aligned}
\beta_{i+4}\left(w a_{1} a_{2} \cdots a_{i}\right) & =2 \cdot \beta_{(i-1)+4}\left(w a_{1} a_{2} \cdots a_{i-1}\right)-\beta_{(i-2)+4}\left(w a_{1} a_{2} \cdots a_{i-2}\right) \\
& =2 \cdot(2 \cdot(i-1)+3)-(2 \cdot(i-2)+3)=2 i+3
\end{aligned}
$$

Second Item. Let us proceed by induction on $i$. It follows from Item (i) that, for $i \geq 1$, Item (ii) is true for $i=1$ and $k=0$. Assume that Item (ii) is true up to $i-1$ for any $0 \leq k \leq i-2$. Then we have, for $1 \leq k \leq i-1$ and for any $w$ in $\bigcup_{n \geq 1} J_{n}$, the following relation:

$$
\begin{aligned}
\beta_{i+4}\left(w a_{1} a_{2} \cdots a_{i-k} a_{0}^{k}\right) & =\beta_{(i-1)+4}\left(w a_{1} a_{2} \cdots a_{(i-1)-(k-1)} a_{0}^{k-1}\right)+1 \\
& =2 \cdot(i-1)-(k-1)+3=2 i-k+3
\end{aligned}
$$

Since, by Item (i), Item (ii) is true for $k=0$, it follows that Item (ii) holds for any $i \geq 1$ and any $0 \leq k \leq i-1$.

Third Item. Item (iii) is true if $i=1$ because $\beta_{5}\left(w c_{1}\right)=4$ for any word $w$ in $\bigcup_{n \geq 1} J_{n}$ (recall that $\left.c_{1} \neq a_{1}\right)$. It is also true if $i=0$. Now proceed by induction on $i$. Assume that this Item is true up to $i-1$, then we have either

$$
\begin{aligned}
\beta_{i+4}\left(w c_{1} \cdots c_{i}\right) & =2 \cdot \beta_{(i-1)+4}\left(w c_{1} \cdots c_{i-1}\right)-\beta_{(i-2)+4}\left(w c_{1} \cdots c_{i-2}\right) \\
& =2 \cdot((i-1)+3)-((i-2)+3)=i+3
\end{aligned}
$$

or

$$
\beta_{i+4}\left(w c_{1} \cdots c_{i}\right)=\beta_{(i-1)+4}\left(w c_{1} \cdots c_{i-1}\right)+1=((i-1)+3)+1=i+3
$$

which ends the proof.

Lemma 5.8. Let $i$ be an integer such that $i \geq 5$. Consider two words $w_{1}$ and $w_{2}$ of the Jacquard language $J_{l}$, with $l \geq i-3$, such that:

(i) $\beta_{i}\left(w_{1}\right)>\beta_{i}\left(w_{2}\right)$;

(ii) $\beta_{i-1}\left(w_{1}^{\prime}\right) \geq \beta_{i-1}\left(w_{2}^{\prime}\right)$;

(iii) $\beta_{i}\left(w_{1}\right)-\beta_{i-1}\left(w_{1}^{\prime}\right) \geq \beta_{i}\left(w_{2}\right)-\beta_{i-1}\left(w_{2}^{\prime}\right)$.

Then, for any integer $k \geq 1$ and for any word $w$ such that $w_{1} w$ and $w_{2} w$ belong to $J_{k+l}$, we have $\beta_{i+k}\left(w_{1} w\right)>$ $\beta_{i+k}\left(w_{2} w\right)$.

Proof of Lemma 5.8. Consider two words $w_{1}$ and $w_{2}$ in $J_{l}$, with $l \geq i-3$, that satisfy conditions (i)-(iii). Let $a$ be any letter such that $w_{1} a$ and $w_{2} a$ belong to $J_{l+1}$. Then we have the three following cases:

If $a=a_{0}$ then

$$
\begin{aligned}
& \beta_{i+1}\left(w_{1} a_{0}\right)=\beta_{i}\left(w_{1}\right)+1 \\
& \beta_{i+1}\left(w_{2} a_{0}\right)=\beta_{i}\left(w_{2}\right)+1 .
\end{aligned}
$$

If $a=a_{1}$ then

$$
\begin{aligned}
& \beta_{i+1}\left(w_{1} a_{1}\right)=\beta_{i}\left(w_{1}\right)+\beta_{i-1}\left(w_{1}^{\prime}\right) \\
& \beta_{i+1}\left(w_{2} a_{1}\right)=\beta_{i}\left(w_{2}\right)+\beta_{i-1}\left(w_{2}^{\prime}\right) .
\end{aligned}
$$


If $a=a_{j}$, for $j \geq 2$, then

$$
\begin{aligned}
& \beta_{i+1}\left(w_{1} a_{j}\right)=\beta_{i}\left(w_{1}\right)+\beta_{i}\left(w_{1}\right)-\beta_{i-1}\left(w_{1}^{\prime}\right) \\
& \beta_{i+1}\left(w_{2} a_{j}\right)=\beta_{i}\left(w_{2}\right)+\beta_{i}\left(w_{2}\right)-\beta_{i-1}\left(w_{2}^{\prime}\right) .
\end{aligned}
$$

Therefore, in any case, the words $w_{1} a$ and $w_{2} a$ satisfy the three conditions (i-iii); and in particular, we have $\beta_{i+1}\left(w_{1} a\right)>\beta_{i+1}\left(w_{2} a\right)$. Hence the lemma is true for $k=1$. An induction argument on the length of $w$, based on the same relations as for $k=1$, ends the proof.

Proof of Theorem 5.6. By Theorem 5.4, if two Goursat structures have the same singularity types at $p$ and $\tilde{p}$, respectively, then they have the same growth vector at $p$ and $\tilde{p}$, respectively. Now, we will prove the converse. Suppose that $w$ and $\tilde{w}$ are the singularity types of two distributions $\mathcal{D}$ and $\tilde{\mathcal{D}}$ at $p$ and $\tilde{p}$, respectively, that is $w=\delta_{\mathcal{D}}(p)$ and $\tilde{w}=\delta_{\mathcal{D}}(p)$. We will show that if $w \neq \tilde{w}$ then there exists an integer $i_{0}$ such that $\beta_{i_{0}}(w) \neq \beta_{i_{0}}(\tilde{w})$.

It is easy to check that if $w$ and $\tilde{w}$ are two words of the Jacquard language $J_{n}$ such that $w \neq \tilde{w}$ then there exists (after a permutation of $w$ and $\tilde{w}$, if necessary) three words $z, v$, and $\tilde{v}$ such that both $w=v z$ and $\tilde{w}=\tilde{v} z$, and which satisfy either

$$
\left\{\begin{array}{l}
v=u a_{1} a_{2} \cdots a_{i-k} a_{0}^{k} \\
\tilde{v}=\tilde{u} c_{1} c_{2} \cdots c_{i}
\end{array}\right.
$$

where $0 \leq k \leq i-1$ and $c_{j} \neq a_{1}$ for $1 \leq j \leq i$, or

$$
\left\{\begin{array}{l}
v=u a_{1} a_{2} \cdots a_{i-k} a_{0}^{k} \\
\tilde{v}=\tilde{u} a_{1} a_{2} \cdots a_{i-l} a_{0}^{l}
\end{array}\right.
$$

where $k \neq l$.

For each of these two cases we can apply Lemma 5.7. In the first case we have $\beta_{i+4}(v)=2 i-k+3$; while $\beta_{i+4}(\tilde{v})=i+3$. Since $k \leq i-1$ we have $\beta_{i+4}(v) \neq \beta_{i+4}(\tilde{v})$. In the second case we have $\beta_{i+4}(v)=2 i-k+3$; while $\beta_{i+4}(\tilde{v})=2 i-l+3$. Since $k \neq l$ we have $\beta_{i+4}(v) \neq \beta_{i+4}(\tilde{v})$. Therefore, in both cases, we have $\beta_{i+4}(v) \neq \beta_{i+4}(\tilde{v})$; but $\beta_{i+3}(v)=\beta_{i+3}(\tilde{v})$, since by the Item (iii) of Lemma 5.7 they are both equal to $i+2$. Put $i_{0}=(i+4)+|z|$. By Lemma 5.8, we have $\beta_{i_{0}}(w) \neq \beta_{i_{0}}(\tilde{w})$.

\subsection{Computing the singularity type}

Up to now, we have worked with a definition of the singularity type that uses the submanifolds $S_{j}^{(i)}$. Although being geometric, that is independent of a description of the Goursat structure in particular coordinates, it does not tell us how to compute this invariant (unless we know how to compute all $S_{j}^{(i)}$ explicitly). In order to fill this gap we give the following proposition, which yields to a constructive procedure to compute the singularity type of any Goursat structure in terms of its growth vector. Its proof is straightforward.

Proposition 5.9. Let $\mathcal{D}$ be a Goursat structure considered in a neighborhood of a point $p$ that belongs to a manifold of dimension $n \geq 5$. For $0 \leq i \leq n-5$ and $1 \leq j \leq i$, the point $p$ belongs to $S_{j}^{(i)}$ if and only if the growth vector at $p$ of the distribution $\mathcal{D}^{(i-j)}$ starts with

$$
(i-j+2, i-j+3, \ldots, i+2, i+3, i+4, \ldots, i+4, i+5),
$$

where the integer $i+4$ is repeated $j+2$ times. 


\section{Abnormal CURVES}

We proved in the last section that the singularity type and the growth vector contain the same information about a given Goursat structure. That information is, however, encoded in the singularity type in a more systematic and much more geometric way. An illustration of the latter claim is this section in which we study abnormal curves of Goursat structures and we show that the submanifolds $S_{j}^{(i)}$, used to define the singularity type, determine all abnormal curves of any element of the derived flag of a Goursat structure. A precise statement of that result is given as Theorem 6.2 and is followed by a description of the geometry of abnormal curves and by an illustrative example in $\mathbb{R}^{7}$. A proof of Theorem 6.2 is given in Appendix B. In Section 6.3, we prove that two Goursat structures have the same singularity type at a given point if and only if the distributions of their derived flags have the same abnormal curves, up to a local diffeomorphism. Our study of abnormal curves (Th. 6.6) and our analysis, performed in Section 5, of the growth vector and singularity type (Th. 5.6) are summarized in the Introduction as Theorem 1, which gives one of the main contributions of the paper. Roughly speaking, this theorem says that the local information about a Goursat structure encoded in the growth vector, in the singularity type, and in abnormal curves, of all elements of the derived flag, coincide. In the two last sections we study rigid curves of Goursat structures. Although rigid curves are always abnormal, abnormal curves are not always rigid. We will prove that in the case of Goursat structures these two concepts coincide (for $C^{1}$ immersed curves). In the last subsection we will calculate rigid curves of the $n$-trailer and give their natural mechanical interpretation: they correspond to motions that fix the positions of the centers of at least two trailers.

\subsection{Integral and abnormal curves}

Let $M$ be a smooth manifold of dimension $n$ and let $\mathcal{A}$ be a set-valued map $\mathcal{A}: M \rightarrow T M$ such that $\mathcal{A}(p) \subset T_{p} M$, for each point $p$ in $M$. Note that we do not ask $\mathcal{A}(p)$ to be a linear subspace of $T_{p} M$, but just a subset of $T_{p} M$. Neither we ask $\mathcal{A}$ to be smooth. An integral curve of $\mathcal{A}$ is an absolutely continuous map $x: I \rightarrow M$, from an interval $I \subset \mathbb{R}$ to $M$, such that $\dot{x}(t)$ belongs to $\mathcal{A}(x(t))$ for almost all $t$ in $I$. A nontrivial lift of $x(\cdot)$ is an absolutely continuous map $P: I \rightarrow T^{*} M$ such that $P(t)$ belongs to $T_{x(t)}^{*} M$ and $P(t) \neq 0$ for each $t$ in $I$.

Locally, all integral curves of a rank $k$ distribution $\mathcal{D}=\left(f_{1}, \ldots, f_{k}\right)$ can be described as solutions of an (underdetermined) ordinary differential equation. Indeed, for any given integral curve $x(\cdot)$ of $\mathcal{D}$ we can clearly find $k$ real-valued measurable functions $u_{i}$, for $1 \leq i \leq k$, such that

$$
\dot{x}(t)=\sum_{i=1}^{k} f_{i}(x(t)) u_{i}(t)
$$

holds for almost all $t$ in $I$. These functions $u_{i}$ are called controls. Observe that the controls associated to an integral curve are not uniquely defined. In control theory, an overdetermined differential equation of the form (34), where the functions $u_{i}$ for $1 \leq i \leq k$ can be taken as arbitrary measurable functions, is called a control system. Informally, the system (34) can be seen as a "parametrization" of the set of all integral curves of $\mathcal{D}$ by $k$ real-valued measurable functions.

Roughly speaking, a solution $x(\cdot)$ of (34) is abnormal if it is a singular point of the end-point mapping or, equivalently, if the linearization of the control system along $x(\cdot)$ is not controllable. Many equivalent definitions of the concept of abnormal curves are available (see e.g. the papers [1,3,7,67,77], the survey article [45], and the references given there). The definition that we will use is the one that appears in Pontryagin's Maximum principle [59]. For further details, we refer the reader to the above mentioned works.

Since the results of this section will be local we can work in a coordinate chart $x: M \rightarrow \mathbb{R}^{n}$. Denote by $(x, p)$ the corresponding coordinates on $T^{*} M$. In these coordinates, the Hamiltonian of the control system $(34)$ 
associated to a distribution $\mathcal{D}=\left(f_{1}, \ldots, f_{k}\right)$ is the function defined on $\mathbb{R}^{n} \times \mathbb{R}^{n} \times \mathbb{R}^{k}$ by

$$
H(x, p, u)=\left\langle p, \sum_{i=1}^{k} f_{i}(x) u_{i}\right\rangle,
$$

where both $x$ and $p$ belong to $\mathbb{R}^{n}$ and $u=\left(u_{1}, \ldots, u_{k}\right)$ belongs to $\mathbb{R}^{k}$ and $\langle\cdot, \cdot\rangle$ denotes the pairing between vector fields and differential forms.

Definition 6.1. An integral curve $x: I \rightarrow \mathbb{R}^{n}$, corresponding to a measurable control $u: I \rightarrow \mathbb{R}^{k}$, of the control system $(34)$ is called abnormal if it admits a nontrivial lift $(x(\cdot), p(\cdot))$ such that

$$
\begin{aligned}
\dot{x}(t) & =\frac{\partial H(x(t), p(t), u(t))}{\partial p} \\
\dot{p}(t) & =-\frac{\partial H(x(t), p(t), u(t))}{\partial x}
\end{aligned}
$$

and

$$
\frac{\partial H(x(t), p(t), u(t))}{\partial u}=0
$$

for almost all $t$ in $I$.

By definition, an integral curve of a distribution $\mathcal{D}=\left(f_{1}, \ldots, f_{k}\right)$ is abnormal if it is an abnormal curve of the corresponding control system. It is well known that the abnormal curves of $\mathcal{D}$ depend neither on the choice of coordinates nor on the vector fields $f_{1}, \ldots, f_{k}$ chosen to span the distribution.

Let $I \subset \mathbb{R}$ be an interval. For any $t_{0} \in I$ and for any $\varepsilon>0$, denote by $I_{\varepsilon}\left(t_{0}\right)$ the intersection $I \cap\left[t_{0}-\varepsilon, t_{0}+\varepsilon\right]$. An integral curve $x: I \rightarrow M$ is locally abnormal if for each $t_{0}$ in $I$ there exists a small enough $\varepsilon>0$ such that the restriction of $x(\cdot)$ to $I_{\varepsilon}\left(t_{0}\right)$ is abnormal.

\subsection{Abnormal curves of Goursat structures}

Let $\mathcal{D}$ be a Goursat structure on a manifold $M$ of dimension $n \geq 3$. Recall that its singularity type can be computed using the sequence of canonical manifolds defined, for $0 \leq i \leq n-5$, by

$$
S_{0}^{(i)}=\left\{q \in M: \mathcal{D}^{(i)}(q)=\mathcal{C}_{i+1}(q)\right\}
$$

and, for $1 \leq j \leq i$, by

$$
S_{j}^{(i)}=\left\{q \in S_{j-1}^{(i)}: \mathcal{D}^{(i-j)}(q) \cap T_{q} S_{j-1}^{(i)} \neq \mathcal{C}_{i-j}(q)\right\},
$$

where the distributions $\mathcal{C}_{i}$ are the canonical distributions of Proposition 4.1 (see Sect. 4). Assume that for two given non-negative integers $i$ and $j$, such that $0 \leq i+j \leq n-5$ we have $S_{j}^{(i+j)} \neq \emptyset$. In this case, we can define on $S_{j}^{(i+j)}$ a smooth distribution $\mathcal{A}_{j}^{(i)}$ by taking

$$
\mathcal{A}_{j}^{(i)}(q)=\mathcal{D}^{(i)}(q) \cap T_{q} S_{j}^{(i+j)}
$$

for each point $q$ in $S_{j}^{(i+j)}$. It is easy to check, using a Kumpera-Ruiz normal form, that $\mathcal{A}_{j}^{(i)}$ is indeed a smooth distribution and that its rank is $i+1$. Although each $\mathcal{A}_{j}^{(i)}$ is defined only on $S_{j}^{(i+j)}$, we can extend the definition of $\mathcal{A}_{j}^{(i)}$ to $M$ by taking $\mathcal{A}_{j}^{(i)}(q)=0$ for all points $q$ that do not belong to $S_{j}^{(i+j)}$ and thus consider $\mathcal{A}_{j}^{(i)}$ as a 
set valued map defined everywhere on $M$. This extension allows us to define, for any $0 \leq i \leq n-5$, a subset $\mathcal{A}^{(i)} \subset T M$ by

$$
\mathcal{A}^{(i)}(q)=\mathcal{C}_{i}(q) \cup\left(\bigcup_{0 \leq j \leq n-i-5} \mathcal{A}_{j}^{(i)}(q)\right)
$$

for each point $q$ in $M$. Note that, usually, the subset $\mathcal{A}^{(i)} \subset T M$ is not a distribution.

By definition, we take $\mathcal{A}^{(n-4)}=\mathcal{C}_{n-4}$. Moreover, we define $\mathcal{A}^{(n-3)}$ as the characteristic distribution of $\mathcal{D}^{(n-3)}$, which is equal to $\mathcal{C}_{n-4}$ if $n \geq 4$ and equal to $\{0\}$ if $n=3$. Finally, we take $\mathcal{A}^{(n-2)}=\emptyset$. Observe that the set-valued maps $\mathcal{A}^{(n-3)}=\{0\}$ and $\mathcal{A}^{(n-2)}=\emptyset$ are different. Indeed, the first one has trivial integral curves (points); while the second one has no integral curves at all.

Theorem 6.2. Consider a Goursat structure $\mathcal{D}$ defined on a manifold of dimension $n$ and fix an integer $i$ such that $0 \leq i \leq n-2$. An integral curve of $\mathcal{D}^{(i)}$ is locally abnormal if and only if it is an integral curve of $\mathcal{A}^{(i)}$.

For $i=n-4, n-3$, and $n-2$, the distribution $\mathcal{D}^{(i)}$ is of rank $n-2, n-1$, and $n$, respectively, and the proof of Theorem 6.2 follows easily from well known results. Indeed, if $i=n-4$ then the distribution $\mathcal{D}^{(i)}$, which is of rank $n-2$, can be transformed into a direct generalization of Engel's normal form ([32,43,76], and [78]) given by Theorem C.4 (see Appendix C), where we have to take $k=n-2$ and $m=2$. In this case, the abnormal curves of $\mathcal{D}^{(n-4)}$ are clearly the integral curves of $\mathcal{C}_{n-4}$ (see Lem. B.1 below). If $i=n-3$ then the distribution $\mathcal{D}^{(i)}$, which is of rank $n-1$, is annihilated locally by a 1 -form $\omega$ such that $d \omega \wedge \omega \neq 0$ and $(d \omega)^{2} \wedge \omega=0$. This property is equivalent to the fact that the characteristic distribution of $\mathcal{D}^{(n-3)}$ is of corank 2 in $\mathcal{D}^{(n-3)}$ (see [6]), and it implies that $\mathcal{D}^{(n-3)}$ is locally given by the normal form of Theorem C.4, where $k=n-1$ and $m=1$. Note, however, that this form does not follow from Theorem C.4 whose condition, when $m=1$, is only necessary but not sufficient. In this case, it is straightforward to see that the abnormal curves of $\mathcal{D}^{(n-3)}$ are the integral curves of the characteristic distribution of $\mathcal{D}^{(n-3)}$, which is an involutive distribution that has rank $n-3$. Finally, if $i=n-2$ then the situation is even simpler because $\mathcal{D}^{(n-2)}=T M$, which implies that $\mathcal{D}^{(n-2)}$ has no abnormal curves at all. Hence the only values of $i$ that will be considered in the proof of Theorem 6.2 are $0 \leq i \leq n-5$.

In order to explain further the meaning of Theorem 6.2 we would like to emphasize the following points, relative to the geometric structure of $\mathcal{A}^{(i)}$ and its integral curves. These facts follow directly from our study of the singularity type (see Sect. 4) and will be used in the proof of Theorem 6.2.

(i) Although for each point $q$ in $M$ we have, by definition,

$$
\mathcal{A}^{(i)}(q)=\mathcal{C}_{i}(q) \cup\left(\bigcup_{0 \leq j \leq n-i-5} \mathcal{A}_{j}^{(i)}(q)\right),
$$

the relations $S_{k}^{(i+k)} \cap S_{j}^{(i+j)}=\emptyset$ for $k \neq j$ (see Prop. 4.2) imply that, for a fixed point $q$, only two possibilities can occur. Indeed, we have either

$$
\mathcal{A}^{(i)}(q)=\mathcal{C}_{i}(q) \quad \text { or } \quad \mathcal{A}^{(i)}(q)=\mathcal{C}_{i}(q) \cup \mathcal{A}_{j}^{(i)}(q),
$$

for a unique integer $j$ such that $0 \leq j \leq n-i-5$. In other words, for each point $q$ the subset $\mathcal{A}^{(i)}(q) \subset T_{q} M$ is the union (not the sum!) of either one or two linear subspaces of $T_{q} M$. Note that if $i \geq 1$ then $\mathcal{C}_{i}(q) \cap \mathcal{A}_{j}^{(i)}(q)=$ $\mathcal{C}_{i-1}(q)$.

(ii) For $0 \leq i \leq n-5$, we define the set $K_{i}=\bigcup_{j=i}^{n-5} S_{0}^{(j)}$; for any other value of $i$ we take $K_{i}=\emptyset$. We will call this set the singular locus of $\mathcal{D}^{(i)}$. If $i=n-4, n-3$, or $n-2$ then, by definition, the singular locus is empty, which explains why these cases are simpler. If $i=0$ then this definition agrees with the one given in Section 5 
for the singular locus of $\mathcal{D}$. It follows directly from the properties of the submanifolds $S_{0}^{(j)}$ (see Prop. 4.4) that $K_{i}$ is a stratified manifold. In fact, in Kumpera-Ruiz normal form coordinates, this set is an algebraic variety defined by a single polynomial equation of the form $\prod_{r=0}^{m-1} x_{k_{r}}^{r}=0$, where the integer $m$ corresponds to the number of singularities of $\mathcal{D}^{(i)}$, which is, in general, smaller than the number of singularities of $\mathcal{D}$. For any point $q$ that does not belong to $K_{i}$ we clearly have $\mathcal{A}^{(i)}(q)=\mathcal{C}_{i}(q)$. Note, however, that there exist points of $K_{i}$ for which we also have $\mathcal{A}^{(i)}(q)=\mathcal{C}_{i}(q)$.

(iii) For $0 \leq i \leq n-5$, define the set $L_{i}=\bigcup_{j=0}^{n-i-5} S_{j}^{(i+j)}$. Since for any $j$ we have $S_{j}^{(i)} \subset S_{0}^{(i)}$, it follows that $L_{i} \subset K_{i}$. For $0 \leq i \leq n-5$, the set of points such that $\mathcal{A}^{(i)}(q) \neq \mathcal{C}_{i}(q)$ is precisely $L_{i}$. In other words, the set $\mathcal{A}^{(i)}(q)$ is a linear subspace of $T_{q} M$ if and only if $q$ does not belong to $L_{i}$. Unlike $K_{i}$, the set $L_{i}$ is always a smooth submanifold of $M$. Note, however, that $L_{i}$ can have several connected components and that the dimensions of these components can be different. Nevertheless, in a small enough neighborhood $U$ of any of its points, the submanifold $L_{i}$ is connected and coincides with one and only one of the smooth manifolds $S_{j}^{(i+j)} \cap U$.

For example, in the case of a distribution spanned by a Kumpera-Ruiz normal form on $\mathbb{R}^{n}$, the set $L_{i}$ is connected. If non-empty, the set $L_{i}$ is a codimension $j+1$ linear subspace of $\mathbb{R}^{n}$, where $j$ is the only integer such that $S_{j}^{(i+j)}$ is non-empty. In the case of the $n$-trailer system, the situation is quite different. For example, for the two-trailer system, the submanifold $L_{0}$ has two connected components, given by $\left\{\theta_{2}-\theta_{1}=\pi / 2\right\}$ and $\left\{\theta_{2}-\theta_{1}=-\pi / 2\right\}$. Each of them has codimension 1. For the three-trailer system, the submanifold $L_{0}$ has six connected components given, respectively, by $\left\{\theta_{3}-\theta_{2}=\pi / 2\right\},\left\{\theta_{3}-\theta_{2}=-\pi / 2\right\},\left\{\theta_{3}-\theta_{2}=\pi / 4 ; \theta_{2}-\theta_{1}=\pi / 2\right\}$, $\left\{\theta_{3}-\theta_{2}=-3 \pi / 4 ; \theta_{2}-\theta_{1}=\pi / 2\right\},\left\{\theta_{3}-\theta_{2}=-\pi / 4 ; \theta_{2}-\theta_{1}=-\pi / 2\right\},\left\{\theta_{3}-\theta_{2}=3 \pi / 4 ; \theta_{2}-\theta_{1}=-\pi / 2\right\}$. Two of them have codimension 1 ; four of them have codimension 2 .

\subsection{An illustrative example}

We consider now a more detailed example. Let $\mathcal{D}$ be the distribution spanned by the following Kumpera-Ruiz normal form on $\mathbb{R}^{7}$ :

$$
\left(\frac{\partial}{\partial x_{7}},\left(x_{7}+c_{7}\right) \frac{\partial}{\partial x_{6}}+\frac{\partial}{\partial x_{5}}+x_{6}\left(\frac{\partial}{\partial x_{4}}+x_{5}\left(x_{4} \frac{\partial}{\partial x_{3}}+x_{3} \frac{\partial}{\partial x_{2}}+\frac{\partial}{\partial x_{1}}\right)\right)\right),
$$

where $c_{7}$ is either equal to 0 or 1 . When $c_{7}=1$, the singularity type of $\mathcal{D}$ at zero is $a_{0} a_{1} a_{1} a_{0}$ and the growth vector at zero is $(2,3,4,5,5,6,6,6,7)$; while when $c_{7}=0$, the singularity type is $a_{0} a_{1} a_{1} a_{2}$ and the growth vector $(2,3,4,5,5,5,6,6,6,6,7)$.

In both cases, we have

$$
S_{0}^{(0)}=\emptyset, S_{0}^{(1)}=\left\{x_{6}=0\right\}, \quad \text { and } \quad S_{0}^{(2)}=\left\{x_{5}=0\right\} .
$$

Therefore, the singular loci of the distributions $\mathcal{D}^{(0)}, \mathcal{D}^{(1)}$, and $\mathcal{D}^{(2)}$ are given respectively by

$$
K_{0}=\left\{x_{6} x_{5}=0\right\}, \quad K_{1}=\left\{x_{6} x_{5}=0\right\}, \quad \text { and } \quad K_{2}=\left\{x_{5}=0\right\}
$$

If $c_{7}=1$ then, in a small enough neighborhood of zero, we have $S_{1}^{(1)}=\emptyset$; but if $c_{7}=0$ then we have $S_{1}^{(1)}=\left\{x_{7}=x_{6}=0\right\}$. In both cases we have $S_{1}^{(2)}=\emptyset$.

If $c_{7}=1$ then, in a small enough neighborhood $U$ of zero, we have $\mathcal{A}^{(0)}=\mathcal{C}_{0}=\left(\frac{\partial}{\partial x_{7}}\right)$, which is a smooth distribution on $U$; but if $c_{7}=0$ then the subset $\mathcal{A}^{(0)}$ coincides with the smooth distribution $\mathcal{C}_{0}=\left(\frac{\partial}{\partial x_{7}}\right)$ outside $L_{0}=\left\{x_{7}=x_{6}=0\right\}$ while for any point $p$ of $L_{0}$ we have

$$
\mathcal{A}^{(0)}(p)=\left(\frac{\partial}{\partial x_{7}}\right)(p) \cup\left(\frac{\partial}{\partial x_{5}}\right)(p),
$$


which is clearly not a distribution. In both cases, we have $\mathcal{A}^{(1)}=\mathcal{C}_{1}=\left(\frac{\partial}{\partial x_{7}}, \frac{\partial}{\partial x_{6}}\right)$ outside $L_{1}=\left\{x_{6}=0\right\}$ while for any point $p$ of $L_{1}$ we have

$$
\mathcal{A}^{(1)}(p)=\left(\frac{\partial}{\partial x_{7}}, \frac{\partial}{\partial x_{6}}\right)(p) \cup\left(\frac{\partial}{\partial x_{7}}, \frac{\partial}{\partial x_{5}}\right)(p)
$$

Finally, we have $\mathcal{A}^{(2)}=\left(\frac{\partial}{\partial x_{7}}, \frac{\partial}{\partial x_{6}}, \frac{\partial}{\partial x_{5}}\right)$ outside $L_{2}=\left\{x_{5}=0\right\}$ while for any point $p$ of $L_{2}$ we have

$$
\mathcal{A}^{(2)}(p)=\left(\frac{\partial}{\partial x_{7}}, \frac{\partial}{\partial x_{6}}, \frac{\partial}{\partial x_{5}}\right)(p) \cup\left(\frac{\partial}{\partial x_{7}}, \frac{\partial}{\partial x_{6}}, \frac{\partial}{\partial x_{4}}\right)(p) .
$$

\subsection{Abnormal curves and singularity type}

Theorem 6.3. Let $\mathcal{D}$ and $\tilde{\mathcal{D}}$ be two Goursat structures defined respectively on two manifolds $M$ and $\tilde{M}$, both of dimension n. Fix two points $p$ and $\widetilde{p}$ of $M$ and $\tilde{M}$, respectively. There exists a diffeomorphism $\varphi$, with $\tilde{p}=\varphi(p)$, between two small enough neighborhoods of $p$ and $\tilde{p}$ that transforms, for $0 \leq i \leq n-4$, the abnormal curves of $\mathcal{D}^{(i)}$ into the abnormal curves of $\tilde{\mathcal{D}}^{(i)}$ if and only if the singularity type of $\mathcal{D}$ at $p$ equals the singularity type of $\tilde{\mathcal{D}}$ at $\tilde{p}$.

Proof of Theorem 6.3. Necessity: Consider two distributions $\mathcal{D}$ and $\tilde{\mathcal{D}}$, defined on two manifolds $M$ and $\tilde{M}$, respectively, that have different singularity types $w$ and $\tilde{w}$ at $p$ and $\tilde{p}$, respectively, that is $w=\delta_{\mathcal{D}}(p)$ and $\tilde{w}=\delta_{\tilde{\mathcal{D}}}(\tilde{p})$. We have already pointed out (see the proof of Th. 5.6) that if $w$ and $\tilde{w}$ are two words of the Jacquard language $J_{n}$ such that $w \neq \tilde{w}$ then there exists (after a permutation of $w$ and $\tilde{w}$, if necessary) three words $z, v$, and $\tilde{v}$ such that both $w=v z$ and $\tilde{w}=\tilde{v} z$, and which satisfy either

$$
\left\{\begin{array}{l}
v=u a_{1} a_{2} \cdots a_{i-k} a_{0}^{k} \\
\tilde{v}=\tilde{u} c_{1} c_{2} \cdots c_{i}
\end{array}\right.
$$

where $0 \leq k \leq i-1$ and $c_{j} \neq a_{1}$ for $1 \leq j \leq i$, or

$$
\left\{\begin{array}{l}
v=u a_{1} a_{2} \cdots a_{i-k} a_{0}^{k} \\
\tilde{v}=\tilde{u} a_{1} a_{2} \cdots a_{i-l} a_{0}^{l}
\end{array}\right.
$$

where $k<l$.

In both cases, consider the abnormal curves of $\mathcal{D}^{\left(i_{0}+k\right)}$ and $\tilde{\mathcal{D}}^{\left(i_{0}+k\right)}$, where $i_{0}=|z|$. It follows directly from the definition of the singularity type (see Def. 4.3) that for $\mathcal{D}$ we have $p \in S_{i-k-1}^{\left(i_{0}+i-1\right)}$ while for $\tilde{\mathcal{D}}$ the point $\tilde{p}$ does not belong to any submanifold $S_{j}^{\left(i_{0}+k+j\right)}$. Therefore, the subset $\mathcal{A}^{\left(i_{0}+k\right)}(p)$ is not a linear subspace of $T_{p} M$ while the subset $\tilde{\mathcal{A}}^{\left(i_{0}+k\right)}(\tilde{p})$ is a linear subspace of $T_{\tilde{p}} \tilde{M}$. For each vector $\tau_{p}$ of $\mathcal{A}^{\left(i_{0}+k\right)}(p)$ there exists an abnormal curve of $\mathcal{D}^{\left(i_{0}+k\right)}$ that is tangent to $\tau_{p}$; for each vector $\tilde{\tau}_{p}$ of $\tilde{\mathcal{A}}^{\left(i_{0}+k\right)}(\tilde{p})$ there exists an abnormal curve of $\tilde{\mathcal{D}}^{\left(i_{0}+k\right)}$ that is tangent to $\tilde{\tau}_{p}$. It follows that no diffeomorphism can transform the abnormal curves of $\mathcal{D}^{\left(i_{0}+k\right)}$ into the abnormal curves of $\tilde{\mathcal{D}}^{\left(i_{0}+k\right)}$, locally at $p$ and $\tilde{p}$.

Sufficiency: Now, assume that the singularity type $\delta_{\mathcal{D}}(p)$ of $\mathcal{D}$ at $p$ and $\delta_{\tilde{\mathcal{D}}}(\tilde{p})$ of $\tilde{\mathcal{D}}$ at $\tilde{p}$ coincide and are equal to $w$. The distribution $\mathcal{D}$ (respectively $\tilde{\mathcal{D}}$ ) can be converted into a Kumpera-Ruiz normal form $\kappa^{n}$ (respectively $\tilde{\kappa}^{n}$ ) centered at $p$ (respectively $\tilde{p}$ ) via a diffeomorphisms $\phi$ (respectively $\tilde{\phi}$ ). Let $x=\left(x_{1}, \ldots, x_{n}\right.$ ) (respectively $\tilde{x}=\left(\tilde{x}_{1}, \ldots, \tilde{x}_{n}\right)$ ) denote the coordinates in which $\kappa^{n}$ (respectively $\tilde{\kappa}^{n}$ ) is expressed. By Corollary 4.6 and the invariance of the singularity type we have $\delta_{\kappa^{n}}=\delta_{\tilde{\kappa}^{n}}=w$. Moreover, by Proposition 4.4, a submanifold $S_{j}^{(i+j)}$ contains zero if and only if the submanifold $\tilde{S}_{j}^{(i+j)}$ contains zero, which is the case if and only if 
$w=w_{1} a_{1} \cdots a_{j+1} w_{2}$, for some words $w_{1}$ and $w_{2}$ such that $\left|w_{2}\right|=i$. If those manifolds contain zero then, once again by Proposition 4.4, they are respectively given by

$$
S_{j}^{(i+j)}=\left\{x_{n-i-j}=0, \ldots, x_{n-i}=0\right\} \quad \text { and } \quad \tilde{S}_{j}^{(i+j)}=\left\{\tilde{x}_{n-i-j}=0, \ldots, \tilde{x}_{n-i}=0\right\} .
$$

Now, for each integer $i$, we must distinguish two cases. First case: If for each integer $j$ the submanifolds $S_{j}^{(i+j)}$ and $\tilde{S}_{j}^{(i+j)}$ are empty, in a small enough neighborhood of zero, then, by Theorem 6.2 , the abnormal curves of $\mathcal{D}^{(i)}$ (respectively $\tilde{\mathcal{D}}^{(i)}$ ) are, in a small enough neighborhood of zero, the integral curves of $\mathcal{C}_{i}$ (respectively $\tilde{\mathcal{C}}_{i}$ ). Moreover, we have

$$
\mathcal{C}_{i}=\left(\frac{\partial}{\partial x_{n}}, \ldots, \frac{\partial}{\partial x_{n-i}}\right) \text { and } \quad \tilde{\mathcal{C}}_{i}=\left(\frac{\partial}{\partial \tilde{x}_{n}}, \ldots, \frac{\partial}{\partial \tilde{x}_{n-i}}\right)
$$

Second case: If for some integer $j$ the submanifolds $S_{j}^{(i+j)}$ and $\tilde{S}_{j}^{(i+j)}$ contain zero then, by Proposition 4.2, this integer $j$ is unique. By Theorem 6.2, the abnormal curves of $\mathcal{D}^{(i)}$ (respectively $\tilde{\mathcal{D}}^{(i)}$ ) are, in a small enough neighborhood of zero, the integral curves of $\mathcal{A}_{j}^{(i)}$ (respectively $\tilde{\mathcal{A}}_{j}^{(i)}$ ). Moreover, we have

$$
\mathcal{A}_{j}^{(i)}(q)=\left(\frac{\partial}{\partial x_{n}}, \ldots, \frac{\partial}{\partial x_{n-i}}\right)(q) \text { and } \quad \tilde{\mathcal{A}}_{j}^{(i)}(\tilde{q})=\left(\frac{\partial}{\partial \tilde{x}_{n}}, \ldots, \frac{\partial}{\partial \tilde{x}_{n-i}}\right)(\tilde{q}),
$$

for each point $q$ (respectively $\tilde{q}$ ) that does not belong to $S_{j}^{(i+j)}$ (respectively $\tilde{S}_{j}^{(i+j)}$ ), and

$$
\begin{aligned}
& \mathcal{A}_{j}^{(i)}(q)=\left(\frac{\partial}{\partial x_{n}}, \ldots, \frac{\partial}{\partial x_{n-i+1}}, \frac{\partial}{\partial x_{n-i-j-1}}\right)(q) \cup\left(\frac{\partial}{\partial x_{n}}, \ldots, \frac{\partial}{\partial x_{n-i}}\right)(q) \\
& \tilde{\mathcal{A}}_{j}^{(i)}(\tilde{q})=\left(\frac{\partial}{\partial \tilde{x}_{n}}, \ldots, \frac{\partial}{\partial \tilde{x}_{n-i+1}}, \frac{\partial}{\partial \tilde{x}_{n-i-j-1}}\right)(\tilde{q}) \cup\left(\frac{\partial}{\partial \tilde{x}_{n}}, \ldots, \frac{\partial}{\partial \tilde{x}_{n-i}}\right)(\tilde{q}),
\end{aligned}
$$

for each point $q$ (respectively $\tilde{q}$ ) that belongs to $S_{j}^{(i+j)}$ (respectively $\tilde{S}_{j}^{(i+j)}$ ).

Let $\Phi$ be the local diffeomorphism of $\mathbb{R}^{n}$ defined by $\tilde{x}_{i}=x_{i}$, for $1 \leq i \leq n$. In both cases, the diffeomorphism

$$
\varphi=\phi^{-1} \circ \Phi \circ \phi
$$

transforms the integral curves of $\mathcal{A}^{(i)}$ into the integral curves of $\tilde{\mathcal{A}}^{(i)}$, and thus, by Theorem 6.2 , the abnormal curves of $\mathcal{D}^{(i)}$ into the abnormal curves of $\tilde{\mathcal{D}}^{(i)}$.

\subsection{Rigid curves of Goursat structures}

The concept of rigidity for integral curves of distributions was introduced by Bryant and Hsu [7]. Rigid curves are always abnormal but there exist abnormal curves that are not rigid (see e.g. $[1,7,77]$ ). Nevertheless, we will prove that in the case of Goursat structures these two concepts coincide (for $C^{1}$ immersed curves).

Definition 6.4. Let $\mathcal{D}$ be a completely nonholonomic distribution defined on a manifold $M$. Fix a closed interval $[a, b]$ and two points $p$ and $q$ in $M$. Denote by $\mathcal{O}_{p, q}$ the space of all $C^{1}$ integral curves $x:[a, b] \rightarrow M$ of $\mathcal{D}$ such that $x(a)=p$ and $x(b)=q$, endowed with the $C^{1}$-topology. An integral curve $x(\cdot)$ that belongs to $\mathcal{O}_{p, q}$ is rigid if there exists a small enough neighborhood $\mathcal{V}$ of $x(\cdot)$ in $\mathcal{O}_{p, q}$ such that any curve $\widetilde{x}:[a, b] \rightarrow M$ contained in $\mathcal{V}$ is a reparametrization of $x(\cdot)$.

Roughly speaking, a curve $x:[a, b] \rightarrow M$ is rigid if it is an isolated point of $\mathcal{O}_{x(a), x(b)}$. Our study of abnormal curves leads easily to the following result, which characterizes immersed rigid curves. This result gives also, for Goursat structures, a more intuitive view of the concept of abnormal curve. 
Let $I \subset \mathbb{R}$ be a closed interval. For any $t_{0} \in I$ and for any $\varepsilon>0$, denote by $I_{\varepsilon}\left(t_{0}\right)$ the intersection $I \cap\left[t_{0}-\varepsilon, t_{0}+\varepsilon\right]$. An integral curve $x: I \rightarrow M$ of $\mathcal{D}$ is locally rigid if for each $t_{0}$ in $I$ there exists a small enough $\varepsilon>0$ such that the restriction of $x(\cdot)$ to $I_{\varepsilon}\left(t_{0}\right)$ is rigid.

Theorem 6.5. Let $x(\cdot)$ be a $C^{1}$ immersed integral curve of a Goursat structure $\mathcal{D}$, defined on a manifold of dimension $n$. The three following conditions are equivalent:

(i) the curve $x(\cdot)$ is locally abnormal;

(ii) the curve $x(\cdot)$ is locally rigid;

(iii) the curve $x(\cdot)$ is either an integral curve of $\mathcal{C}_{0}$ or an integral curve of $\mathcal{A}_{k_{0}-1}^{(0)}$, for some $1 \leq k_{0} \leq n-4$.

We supposed in this theorem that the integral curve is immersed, which means that its velocity (defined everywhere, since the curve is $C^{1}$ ) never vanishes. This assumption is fundamental. Indeed, an immersed rigid curve can loose its rigidity if we change its parametrization in such a way that it is not immersed anymore (see e.g. [73]). Observe also that the theorem is stated for integral curves of $\mathcal{D}^{(0)}$ but not for those of $\mathcal{D}^{(i)}$, if $i \geq 1$. In fact, the abnormal curves of $\mathcal{D}^{(i)}$ such that their velocity does not belong to $\mathcal{C}_{i-1}$ have only a weaker form of rigidity: all curves that are close enough to them in the $C^{1}$ topology stay in a submanifold of the original manifold. We will consider this situation in a forthcoming work.

Our proof of Theorem 6.5 is mainly based on the ideas introduced by Bryant and Hsu [7] and Zhitomirskiu [77]. In particular, it is a direct consequence of Zhitomirski1's work that the immersed integral curves of $\mathcal{C}_{0}$ are rigid.

To prove the rigidity of the integral curves of $\mathcal{A}_{k_{0}-1}^{(0)}$ we follow the main ideas of [77]. Note, however, that the statement for $\mathcal{A}_{k_{0}-1}^{(0)}$ is not implied by any of the results of [1,7,67], or [77] because Goursat structures are highly non-generic and do not fit into the large categories of (generic) rank two distributions studied in those papers. We would like to point out that, in the particular case of dimension five, the rigidity of the immersed integral curves of $\mathcal{A}_{0}^{(0)}$ was already observed in [49]. Moreover, the equivalence of Items (ii) and (iiii) of Theorem 6.5 has already been announced in [56].

Our proof of Theorem 6.5 will use the following lemma, which will be proved later in Appendix D. The normal form that we introduce in it is analogous to the one used in [77] to prove that the integral curves of $\mathcal{C}_{0}$ are rigid.

Lemma 6.6. Let $\mathcal{D}$ be a Goursat structure on a manifold $M$ of dimension $n \geq 5$. If the singularity type of $\mathcal{D}$ at $p$ is equal to $\delta_{\mathcal{D}}(p)=w a_{1} \cdots a_{k_{0}}$ for some $1 \leq k_{0} \leq n-4$, where $w$ is an arbitrary word of $J_{n-k_{0}-4}$, then $\mathcal{D}$ is locally equivalent at $p$ to the distribution spanned by a pair of vector fields that has the following form:

$$
\begin{aligned}
& \xi_{1}=\frac{\partial}{\partial y_{1}} \\
& \xi_{2}=y_{1} \frac{\partial}{\partial y_{2}}+\cdots+y_{k_{0}} \frac{\partial}{\partial y_{k_{0}+1}}+\frac{\partial}{\partial y_{k_{0}+2}}+\frac{1}{2} y_{k_{0}+1}^{2} \frac{\partial}{\partial y_{k_{0}+3}}+\sum_{i=k_{0}+4}^{n} \varphi_{i}(y) \frac{\partial}{\partial y_{i}},
\end{aligned}
$$

where the coordinates $y_{1}, \ldots, y_{n}$ are centered at $p$. In these coordinates, the canonical submanifold $S_{k_{0}-1}^{\left(k_{0}-1\right)}$ is given by

$$
S_{k_{0}-1}^{\left(k_{0}-1\right)}=\left\{y_{1}=0, \ldots, y_{k_{0}}=0\right\}
$$

Moreover, we have $\mathcal{C}_{0}=\left(\xi_{1}\right)$, for any point $p$ of $\mathbb{R}^{n}$, and $\mathcal{A}_{k_{0}-1}^{(0)}(p)=\left(\xi_{2}\right)(p)$, for any point $p$ of $S_{k_{0}-1}^{\left(k_{0}-1\right)}$.

Proof of Theorem 6.5. It is well known that rigidity implies abnormality (see $[1,7,77]$ ) and thus, that (ii) implies (i). By Theorem 6.2, any abnormal curve of $\mathcal{D}^{(0)}$ is an integral curve of $\mathcal{A}^{(0)}$. Recall that $\mathcal{A}^{(0)}(p)=$ $\mathcal{C}_{0}(p) \cup \mathcal{A}_{k_{0}-1}^{(0)}(p)$, for a unique $1 \leq k_{0} \leq n-4$, and that $\mathcal{C}_{0}(p) \cap \mathcal{A}_{k_{0}-1}^{(0)}(p)=0$. Therefore any $C^{1}$ immersed abnormal curve of $\mathcal{D}^{(0)}$ is either an integral curve of $\mathcal{C}_{0}$ or an integral curve of $\mathcal{A}_{k_{0}-1}^{(0)}$. Hence (i) implies (iii). 
What remains to prove is that if a $C^{1}$ immersed integral curve $y: I_{\varepsilon}\left(t_{0}\right) \rightarrow \mathbb{R}^{n}$ of $\mathcal{D}^{(0)}$ is an integral curve of either $\mathcal{C}_{0}$ or $\mathcal{A}_{k_{0}-1}^{(0)}$ then it is rigid. This result is known [77] for the integral curves of $\mathcal{C}_{0}$. We can thus assume that $y(\cdot)$ is an immersed integral curve of $\mathcal{A}_{k_{0}-1}^{(0)}$ (which then, by definition, stays in $S_{k_{0}-1}^{\left(k_{0}-1\right)}$ ). It follows from Lemma 6.6 that we can find coordinates such that $y(\cdot)$ satisfies $y\left(t_{0}-\varepsilon\right)=0$ and is a solution of the following control system:

$$
\begin{aligned}
\dot{y}_{1} & =u_{1} \\
\dot{y}_{2} & =y_{1} u_{2} \\
& \vdots \\
\dot{y}_{k_{0}+1} & =y_{k_{0}} u_{2} \\
\dot{y}_{k_{0}+2} & =u_{2} \\
\dot{y}_{k_{0}+3} & =\frac{1}{2} y_{k_{0}+1}^{2} u_{2} \\
\dot{y}_{i} & =\varphi_{i}(y) u_{2} \quad \text { for } k_{0}+4 \leq i \leq n,
\end{aligned}
$$

with $u_{1}(t)=0$ (because $y_{1}(t)=0$ on $S_{k_{0}-1}^{\left(k_{0}-1\right)}$ ) and $u_{2}(t) \neq 0$ for each $t$ in $I_{\varepsilon}\left(t_{0}\right)$ (because the curve is immersed). Since the coordinates of Lemma 6.6 are chosen to be centered at $y\left(t_{0}-\varepsilon\right)=0$, from $y_{k_{0}}(t)=0$ we conclude that $y_{k_{0}+1}(t)=0$, and thus that $y_{k_{0}+3}(t)=0$, for each $t$ in $I_{\varepsilon}\left(t_{0}\right)$.

Now, consider a $C^{1}$ immersed integral curve $\tilde{y}: I_{\varepsilon}\left(t_{0}\right) \rightarrow \mathbb{R}^{n}$ of $\mathcal{D}^{(0)}$ that has the same end-points as the curve $y(\cdot)$. In particular, we have $\tilde{y}_{k_{0}+3}\left(t_{0}-\varepsilon\right)=0$ and $\tilde{y}_{k_{0}+3}\left(t_{0}+\varepsilon\right)=0$. By taking a small enough neighborhood of $y(\cdot)$ in $\mathcal{O}_{y\left(t_{0}-\varepsilon\right), y\left(t_{0}+\varepsilon\right)}$ (which is not the same as taking a smaller $\varepsilon>0$ ), we can assume that $\tilde{u}_{2}(t) \neq 0$ for each $t$ in $I_{\varepsilon}\left(t_{0}\right)$, where $\tilde{u}_{1}$ and $\tilde{u}_{2}$ denote the controls for which $\tilde{y}(\cdot)$ is a solution of (35). Without loss of generality, we can assume that $\tilde{u}_{2}(t)>0$ (the proof for $\tilde{u}_{2}(t)<0$ is identical). Since we have $\frac{1}{2} \tilde{y}_{k_{0}+1}^{2} \tilde{u}_{2}(t) \geq 0$ for each $t$ in $I_{\varepsilon}\left(t_{0}\right)$ and both $\tilde{y}_{k_{0}+3}\left(t_{0}-\varepsilon\right)=0$ and $\tilde{y}_{k_{0}+3}\left(t_{0}+\varepsilon\right)=0$, we must have $\tilde{y}_{k_{0}+1}^{2}(t)=0$, for each $t$ in $I_{\varepsilon}\left(t_{0}\right)$. Together with $\tilde{u}_{2}>0$, the latter relation implies that, for $1 \leq i \leq k_{0}+1$, we have $\tilde{y}_{i}(t)=0$ for each $t$ in $I_{\varepsilon}\left(t_{0}\right)$, which clearly implies $\tilde{u}_{1}(t)=0$ for each $t$ in $I_{\varepsilon}\left(t_{0}\right)$. Hence, the curve $\tilde{y}(\cdot)$ is a reparametrization of the original curve $y(\cdot)$. Indeed, these two curves are $C^{1}$ immersed integral curves of $\xi_{2}$ and have the same end points (see [77] for more details about this last point).

\subsection{Rigid curves of the $N$-trailer system}

Let us illustrate Theorem 6.5 by applying it to the $n$-trailer system. Let $\mathcal{D}$ be the Goursat structure spanned by the $n$-trailer system $\tau^{n}$ on $\mathbb{R}^{2} \times\left(S^{1}\right)^{n+1}$. By Proposition 4.9 , we have

$$
\begin{aligned}
S_{j}^{(i)}= & \left\{p \in \mathbb{R}^{2} \times\left(S^{1}\right)^{n+1}: \theta_{n-i}-\theta_{n-i-1}= \pm \frac{\pi}{2} \text { and } \tan \left(\theta_{n-i+k}-\theta_{n-i+k-1}\right)=\sin \left(\theta_{n-i+k-1}-\theta_{n-i+k-2}\right),\right. \\
& \quad \text { for } 1 \leq k \leq j\},
\end{aligned}
$$

for $0 \leq i \leq n-2$ and $0 \leq j \leq i$ (recall that $n$ is the number of trailers, not the dimension of the configuration space!). It obviously follows that

$$
\begin{aligned}
S_{j}^{(j)}= & \left\{p \in \mathbb{R}^{2} \times\left(S^{1}\right)^{n+1}: \theta_{n-j}-\theta_{n-j-1}= \pm \frac{\pi}{2} \text { and } \tan \left(\theta_{n-j+k}-\theta_{n-j+k-1}\right)=\sin \left(\theta_{n-j+k-1}-\theta_{n-j+k-2}\right),\right. \\
& \quad \text { for } 1 \leq k \leq j\},
\end{aligned}
$$


for $0 \leq j \leq n-2$. Each submanifold $S_{j}^{(j)}$ has clearly codimension $j+1$. Moreover, these manifolds are pairwise disjoint. Thus a given point $p$ is either in none of the submanifold $S_{j}^{(j)}$ at all or in one and only one of them.

Recall also that $\mathcal{A}_{j}^{(0)}(p)=\mathcal{D}^{(i)}(p) \cap T_{p} S_{j}^{(j)}$ and that $\mathcal{A}^{(0)}(p)=\mathcal{C}_{0}(p) \cup \mathcal{A}_{j}^{(0)}(p)$, for a unique $0 \leq j \leq n-2$. The canonical line field $\mathcal{C}_{0}$ is given on $\mathbb{R}^{2} \times\left(S^{1}\right)^{n+1}$ by $\left(\frac{\partial}{\partial \theta_{n}}\right)$. A simple computation shows that, on each submanifold $S_{j}^{(j)}$, the line field $\mathcal{A}_{j}^{(0)}$ is given by $\mathcal{A}_{j}^{(0)}=\left(\frac{\partial}{\partial \theta_{n}}+\cdots+\frac{\partial}{\partial \theta_{n-j-1}}\right)$.

By Theorem 6.5, a $C^{1}$ motion of the $n$-trailer for which the velocity never vanishes is rigid if and only if: (i) it is an integral curve of $\mathcal{C}_{0}$ or (ii) it is an integral curve of $\mathcal{A}_{j}^{(0)}$. In the second case, the motion lies in $S_{j}^{(j)}$. In fact, there is an easy way to visualize these rigid trajectories:

Corollary 6.7. An immersed motion of the $n$-trailer system is locally rigid if and only if it fixes the positions in the $\left(\xi_{1}, \xi_{2}\right)$-plane of the centers of the axles of at least two trailers.

For example, there passes through any configuration of $\mathbb{R}^{2} \times\left(S^{1}\right)^{n+1}$ an integral curve of $\mathcal{C}_{0}$. The corresponding motion fixes the positions in the $\left(\xi_{1}, \xi_{2}\right)$-plane of all trailers (we just turn the front wheels). If a configuration is such that $\theta_{n}-\theta_{n-1}= \pm \frac{\pi}{2}$ (it belongs to $S_{0}^{(0)}$ ) then, besides the motions associated to $\mathcal{C}_{0}$, there is an additional motion given by $\mathcal{A}_{0}^{(0)}$ for which the positions in the $\left(\xi_{1}, \xi_{2}\right)$-plane of all trailers, excepted the first one, are fixed. For these motions, the center of the first trailer moves on a circle around the center of the second trailer, which turns with its center fixed (see e.g. Fig. 4). Observe that such a motion is possible if and only if $\theta_{n}-\theta_{n-1}= \pm \frac{\pi}{2}$.

\section{Contact transformations}

In this section we study transformations preserving Goursat structures. Clearly, at regular points, they are contact transformations. Theorem 7.3 describes such transformations in the case of arbitrary points (regular or singular). In Section 7.2, we study the fundamental question of whether Goursat structures are locally determined by their abnormal curves, a property which is shared by most distributions. Using the description of abnormal curves obtained in Section 6, we will show that in the world of Goursat structures this property does not hold if the dimension of the underlying manifold is at least six. Then, we will study the question of whether Goursat structures are determined by abnormal curves of all distributions of their derived flags. In view of the results of Sections 5 and 6 , the latter problem turns out to be that of whether Goursat structures are determined by the singularity type or, equivalently, by the growth vector. It was announced in [18] by Cheaito et al. that the growth vector is not a complete local invariant for Goursat structures if the dimension of the underlying manifold is at least nine. We give a proof of this result in Section 7.2. An alternative proof can be found in [60]. Then we will construct on $\mathbb{R}^{11}$ a continuous family (parametrized by a real number) of locally non-equivalent Goursat structures that have the same singularity type, and thus diffeomorphic collections of abnormal curves for all elements of their derived flags. An analogous example of a continuous family of locally non-equivalent Goursat structures on $\mathbb{R}^{10}$, all of them having the same growth vector, was given in [60]. Our method of proving non-equivalence is based on the characterization of singular contact transformations, given in Section 7.1, and it seems to apply, in general, to different cases than the method of [60].

\subsection{A singular version of Bäcklund's theorem}

Let $\mathcal{D}$ and $\tilde{\mathcal{D}}$ be two Goursat structures defined on two manifolds $M$ and $\tilde{M}$, respectively, of dimension $n \geq 3$. A (generalized) contact transformation (of order $n-2$ ) is a smooth diffeomorphism $\phi$ between $M$ and $\tilde{M}$ such that $\left(\phi_{*} \mathcal{D}\right)(\tilde{p})=\tilde{\mathcal{D}}(\tilde{p})$, for each point $\tilde{p}$ in $\tilde{M}$. Such transformations are called automorphisms in the work of Kumpera and Ruiz [32] (see also [19] and [51]). In a neighborhood of a regular point our definition coincides with the classical definition of a contact transformation on the space $J^{n-2}(\mathbb{R}, \mathbb{R})$ of $(n-2)$-jets of functions that have one dependent and one independent variable (see [6] and [55]). From now on, unless we want to distinguish generalized contact transformations from the classical ones, we will omit the word "generalized". 
Fix two points $p$ and $\tilde{p}$ of $M$ and $\tilde{M}$, respectively. Let $\phi$ be a local contact transformation between $\mathcal{D}$ and $\tilde{\mathcal{D}}$ such that $\phi(p)=\tilde{p}$. Fix two small enough neighborhoods $U$ and $\tilde{U}$ of $p$ and $\tilde{p}$, respectively, such that $\tilde{U}=\phi(U)$ and such that $\mathcal{D}$ on $U$ and $\tilde{\mathcal{D}}$ on $\tilde{U}$ are equivalent to two Kumpera-Ruiz normal forms $\kappa^{n}$ and $\tilde{\kappa}^{n}$ centered at $p$ and $\tilde{p}$, respectively, and defined on two open subsets $x(U)$ and $\tilde{x}(\tilde{U})$ of $\mathbb{R}^{n}$, where $x$ and $\tilde{x}$ denote coordinates that transform the Goursat structures $\mathcal{D}$ and $\tilde{\mathcal{D}}$ into their Kumpera-Ruiz normal forms $\kappa^{n}$ and $\tilde{\kappa}^{n}$, respectively. We can assume, without loss of generality, that the first prolongation (in the sequence of prolongations that define $\kappa^{n}$ and $\left.\tilde{\kappa}^{n}\right)$ is regular. Namely $\kappa^{4}=R_{0}\left(\kappa^{3}\right)$ and $\tilde{\kappa}^{4}=R_{0}\left(\tilde{\kappa}^{3}\right)$. Once such a pair of Kumpera-Ruiz charts $(x, U)$ and $(\tilde{x}, \tilde{U})$ has been fixed, we can associate to the contact transformation $\phi$ a unique contact transformation $\Phi$, between $\kappa^{n}$ on $x(U)$ and $\tilde{\kappa}^{n}$ on $\tilde{x}(\tilde{U})$, by taking

$$
\Phi=\tilde{x} \circ \phi \circ x^{-1} .
$$

In other words $\tilde{x}=(\Phi \circ x) \circ \psi$, where $\psi$ denotes the inverse of the diffeomorphism $\phi$. Observe that, since the Kumpera-Ruiz charts $x$ and $\tilde{x}$ are centered at $p$ and $\tilde{p}$, respectively, we have $\Phi(0)=0$. We will denote by $\Phi_{i}$ the $i^{\text {th }}$ component of $\Phi$.

In the next two propositions we will assume that all the above defined data (the Goursat structures $\mathcal{D}$ and $\tilde{\mathcal{D}}$, the diffeomorphism $\phi$, the coordinates $x$ and $\tilde{x}$, and the Kumpera-Ruiz normal forms $\kappa^{n}$ and $\tilde{\kappa}^{n}$ ) have been fixed and, therefore, that the diffeomorphism $\Phi$ is uniquely defined. The following result is a direct consequence of the obvious relations

$$
\phi_{*}\left(\mathcal{C}_{i}\right)=\tilde{\mathcal{C}}_{i}
$$

for $0 \leq i \leq n-4$, where $\mathcal{C}_{i} \subset \mathcal{D}^{(i)}$ denotes the characteristic distribution of $\mathcal{D}^{(i+1)}$ and $\tilde{\mathcal{C}}_{i} \subset \tilde{\mathcal{D}}^{(i)}$ that of $\tilde{\mathcal{D}}^{(i+1)}$ (see Prop. 4.1).

Proposition 7.1. For each $1 \leq i \leq 3$ we have $\Phi_{i}(x)=\Phi_{i}\left(x_{1}, x_{2}, x_{3}\right)$. For each $4 \leq i \leq n$ we have $\Phi_{i}(x)=$ $\Phi_{i}\left(x_{1}, \ldots, x_{i}\right)$.

Decompose $\mathbb{R}^{n}$ into a direct product $\mathbb{R}^{n}=\mathbb{R}^{i} \times \mathbb{R}^{n-i}$. It follows directly from Proposition 7.1 that for each $3 \leq i \leq n$ we can build a diffeomorphism $\Phi^{(i)}$, between the projection of $x(U)$ on $\mathbb{R}^{i}$ and the projection of $\tilde{x}(\tilde{U})$ on $\mathbb{R}^{i}$, by taking the components $\Phi_{j}$, for $1 \leq j \leq i$, as the components of $\Phi^{(i)}$. Denote by $\Psi^{(i)}$ the inverse of $\Phi^{(i)}$. We obviously have $\Phi^{(n)}=\Phi$. The following result is a direct consequence of Proposition 7.1 and the obvious relations

$$
\phi_{*}\left(\mathcal{D}^{(i)}\right)=\tilde{\mathcal{D}}^{(i)},
$$

which hold for $0 \leq i \leq n-2$. Recall that, by definition, the two Kumpera-Ruiz normal forms $\kappa^{n}$ and $\tilde{\kappa}^{n}$ are given by two sequences of prolongations. We will denote by $\kappa^{3}, \ldots, \kappa^{n}$ and $\tilde{\kappa}^{3}, \ldots, \tilde{\kappa}^{n}$, respectively, the Kumpera-Ruiz normal forms obtained as intermediate steps of these successive prolongations.

Proposition 7.2. There exist four smooth functions, denoted by $\nu_{3}, \eta_{3}, \mu_{3}$, and $\lambda_{3}$, that depend on the coordinates $x_{1}, x_{2}$, and $x_{3}$ only, such that

$$
\begin{aligned}
& \Phi_{*}^{(3)}\left(\kappa_{1}^{3}\right)=\left(\nu_{3} \circ \Psi^{(3)}\right) \tilde{\kappa}_{1}^{3}+\left(\lambda_{3} \circ \Psi^{(3)}\right) \tilde{\kappa}_{2}^{3} \\
& \Phi_{*}^{(3)}\left(\kappa_{2}^{3}\right)=\left(\eta_{3} \circ \Psi^{(3)}\right) \tilde{\kappa}_{1}^{3}+\left(\mu_{3} \circ \Psi^{(3)}\right) \tilde{\kappa}_{2}^{3} .
\end{aligned}
$$

Moreover, for each $i \geq 4$, there exist three smooth functions, denoted by $\nu_{i}, \eta_{i}$, and $\mu_{i}$, that depend on the coordinates $x_{1}, \ldots, x_{i}$ only, such that

$$
\begin{aligned}
& \Phi_{*}^{(i)}\left(\kappa_{1}^{i}\right)=\left(\nu_{i} \circ \Psi^{(i)}\right) \tilde{\kappa}_{1}^{i} \\
& \Phi_{*}^{(i)}\left(\kappa_{2}^{i}\right)=\left(\eta_{i} \circ \Psi^{(i)}\right) \tilde{\kappa}_{1}^{i}+\left(\mu_{i} \circ \Psi^{(i)}\right) \tilde{\kappa}_{2}^{i} .
\end{aligned}
$$


The functions $\nu_{i}, \eta_{i}, \mu_{i}$, and $\lambda_{i}$ are uniquely defined, for each $i \geq 3$, once the diffeomorphism $\Phi$ has been fixed. They obviously satisfy $\left(\nu_{3} \mu_{3}-\lambda_{3} \eta_{3}\right)(0) \neq 0$ and $\left(\nu_{i} \mu_{i}\right)(0) \neq 0$, for $i \geq 4$.

The following result can be considered as a singular version of Bäcklund's theorem [2] (see [55] for a modern approach). It shows that any contact transformation is the "prolongation" of a first order contact transformation. Though the case $n=4$ is classical [7], it seems that our result for $n \geq 5$ is new. Notice that a weaker version of Theorem 7.3 has already been announced in [13]. Independently, an infinitesimal version of Theorem 7.3 has been announced in [48] and proved in [51].

Theorem 7.3. Let $\phi$ be a local (generalized) contact transformation between two Goursat structures $\mathcal{D}$ and $\tilde{\mathcal{D}}$, defined locally at $p$ and $\tilde{p}$, respectively. Let $x$ and $\tilde{x}$ be local coordinates that transform $\mathcal{D}$ and $\tilde{\mathcal{D}}$ into their Kumpera-Ruiz normal forms $\kappa^{n}$ and $\tilde{\kappa}^{n}$, respectively, and let $\delta_{\mathcal{D}}(p)=w_{0} \cdots w_{n-4}$ be the singularity type of $\mathcal{D}$ at $p$, which equals $\delta_{\tilde{\mathcal{D}}}(\tilde{p})$ since $\mathcal{D}$ at $p$ and $\tilde{\mathcal{D}}$ at $\tilde{p}$ are locally equivalent. The constants $c_{i}$ and $\tilde{c}_{i}$ that appear in $\kappa^{n}$ and $\tilde{\kappa}^{n}$, respectively, and the contact transformation $\Phi$ associated to $\phi$ and to the coordinates $x$ and $\tilde{x}$ fulfill the following relations:

(i) the diffeomorphism $\Phi^{(3)}$ is a first order contact transformation and the functions $\nu_{3}, \eta_{3}, \mu_{3}$, and $\lambda_{3}$ are uniquely determined by $\Phi^{(3)}$;

(ii) the diffeomorphism $\Phi^{(4)}$ is uniquely defined by

$$
\begin{aligned}
\Phi_{4}(x) & =\frac{\nu_{3}+x_{4} \eta_{3}}{\mu_{3}+x_{4} \lambda_{3}} \\
\mu_{4} & =\mu_{3}+x_{4} \lambda_{3} \\
\nu_{4} & =\mathrm{L}_{\kappa_{1}^{4}} \Phi_{4}=\left(\mu_{3} \eta_{3}-\lambda_{3} \nu_{3}\right) /\left(\nu_{3}+x_{4} \eta_{3}\right)^{2} \\
\eta_{4} & =\mathrm{L}_{\kappa_{2}^{4}} \Phi_{4}
\end{aligned}
$$

(iii) if $i \geq 5$ and $w_{i-4} \neq a_{1}$ then $\Phi^{(i)}$ is uniquely defined by

$$
\begin{aligned}
\tilde{c}_{i} & =c_{i} \frac{\nu_{i-1}(0)}{\mu_{i-1}(0)}+\frac{\eta_{i-1}(0)}{\mu_{i-1}(0)} \\
\Phi_{i}(x) & =\frac{1}{\mu_{i-1}}\left(\left(x_{i}+c_{i}\right) \nu_{i-1}+\eta_{i-1}\right)-\tilde{c}_{i} \\
\mu_{i} & =\mu_{i-1} \\
\nu_{i} & =\mathrm{L}_{\kappa_{1}^{i}} \Phi_{i}=\nu_{i-1} / \mu_{i-1} \\
\eta_{i} & =\mathrm{L}_{\kappa_{2}^{i}} \Phi_{i} ;
\end{aligned}
$$

(iv) if $i \geq 5$ and $w_{i-4}=a_{1}$ then $\Phi^{(i)}$ is uniquely defined by

$$
\begin{aligned}
\Phi_{i}(x) & =\frac{x_{i} \mu_{i-1}}{\nu_{i-1}+x_{i} \eta_{i-1}} \\
\mu_{i} & =\nu_{i-1}+x_{i} \eta_{i-1} \\
\nu_{i} & =\mathrm{L}_{\kappa_{1}^{i}} \Phi_{i}=\left(\mu_{i-1} \nu_{i-1}\right) /\left(\nu_{i-1}+x_{i} \eta_{i-1}\right)^{2} \\
\eta_{i} & =\mathrm{L}_{\kappa_{2}^{i}} \Phi_{i} .
\end{aligned}
$$

Therefore, the (generalized) contact transformation $\Phi$ is uniquely determined by the first order contact transformation $\Phi^{(3)}$.

This theorem says that any (generalized) contact transformation between two Goursat structures is uniquely defined by a first order contact transformation $\Phi^{(3)}$, and by the singularity type and constants of the chosen Kumpera-Ruiz normal forms. In fact, the component $\Phi_{4}$ of $\Phi^{(4)}$ is a linear fractional transformation (Möbius 
transformation) whose coefficients are uniquely determined by the components of $\Phi^{(3)}$ (compare [7]). For $i \geq 5$, successively, the component $\Phi_{i}$ of $\Phi^{(i)}$ is either, in the case of a singular prolongation, a zero preserving linear fractional transformation with $x_{i}=0$ being fixed by the fact that $\Phi^{(i)}$ preserves the hypersurface $\left\{x_{i}=0\right\}$ or, in the case of a regular prolongation, by an affine transformation. In both cases, the coefficients of the linear fractional transformation or of the affine transformation are uniquely determined by $\Phi^{(i-1)}$ and by the singularity type and constants of the chosen Kumpera-Ruiz normal forms.

Proof of Theorem 7.3. If $n=3$ then there is nothing to prove. If $n=4$ then the result is well known (see e.g. [7]). Therefore, we can proceed by induction on the integer $n \geq 5$. Assume that the theorem is true for $n-1$. By Proposition 7.2, we have

$$
\begin{aligned}
& \Phi_{*}^{(n-1)}\left(\kappa_{1}^{n-1}\right)=\left(\nu_{n-1} \circ \Psi^{(n-1)}\right) \tilde{\kappa}_{1}^{n-1} \\
& \Phi_{*}^{(n-1)}\left(\kappa_{2}^{n-1}\right)=\left(\eta_{n-1} \circ \Psi^{(n-1)}\right) \tilde{\kappa}_{1}^{n-1}+\left(\mu_{n-1} \circ \Psi^{(n-1)}\right) \tilde{\kappa}_{2}^{n-1} .
\end{aligned}
$$

In other words, the restriction of $\Phi$ to $x(U) \cap \mathbb{R}^{n-1}$, equipped with coordinates $x_{1}, \ldots, x_{n-1}$, is a contact transformation between $\kappa^{n-1}$ and $\tilde{\kappa}^{n-1}$. Since the theorem is assumed to be true for $n-1$, each component $\Phi_{i}$, for $1 \leq i \leq n-1$, satisfies the relations given by the theorem, as do, for $3 \leq i \leq n-1$, the smooth functions $\nu_{i}, \mu_{i}, \lambda_{i}$, and $\eta_{i}$, given by Proposition 7.2. What remains to check is that $\Phi_{n}, \nu_{n}, \mu_{n}$, and $\eta_{n}$ satisfy our conditions.

Recall that for any diffeomorphism $\Phi^{(n)}=\left(\Phi^{(n-1)}, \Phi_{n}\right)^{\top}$ of $\mathbb{R}^{n}$, such that $\Phi^{(n-1)}$ depends on the first $n-1$ coordinates $x_{1}, \ldots, x_{n-1}$ only, and for any vector field $f=\alpha f^{n-1}+f_{n}$ on $\mathbb{R}^{n}$, where $\alpha$ is a smooth function on $\mathbb{R}^{n}$, the vector field $f^{n-1}$ is the lift of a vector field on $\mathbb{R}^{n-1}$, and the only non-zero component of $f_{n}$ is the last one, we have:

$$
\Phi_{*}^{(n)}(f)=\left(\alpha \circ \Psi^{(n)}\right) \Phi_{*}^{(n-1)}\left(f^{n-1}\right)+\left(\left(\mathrm{L}_{f} \Phi_{n}\right) \circ \Psi^{(n)}\right) \frac{\partial}{\partial \tilde{x}_{n}}
$$

Observe that the vector field $\Phi_{*}^{(n-1)}\left(f^{n-1}\right)$ is lifted (see Notation 2.1) along the coordinate $\tilde{x}_{n}$, which is given by $\Phi_{n}$.

Regular case: If $w_{n-4} \neq a_{1}$ then we have $\kappa_{2}^{n}=\left(x_{n}+c_{n}\right) \kappa_{1}^{n-1}+\kappa_{2}^{n-1}$. This relation, together with (36) and the induction hypothesis leads to:

$$
\begin{aligned}
\Phi_{*}^{(n)}\left(\kappa_{2}^{n}\right) & =\left(\left(x_{n}+c_{n}\right) \circ \Psi^{(n)}\right) \Phi_{*}^{(n-1)}\left(\kappa_{1}^{n-1}\right)+\Phi_{*}^{(n-1)}\left(\kappa_{2}^{n-1}\right)+\left(\left(\mathrm{L}_{\kappa_{2}^{n}} \Phi_{n}\right) \circ \Psi^{(n)}\right) \tilde{\kappa}_{1}^{n} \\
& =\left(\left(\left(x_{n}+c_{n}\right) \nu_{n-1}+\eta_{n-1}\right) \circ \Psi^{(n)}\right) \tilde{\kappa}_{1}^{n-1}+\left(\mu_{n-1} \circ \Psi^{(n)}\right) \tilde{\kappa}_{2}^{n-1}+\left(\left(\mathrm{L}_{\kappa_{2}^{n}} \Phi_{n}\right) \circ \Psi^{(n)}\right) \tilde{\kappa}_{1}^{n} \\
& =\left(\mu_{n-1} \circ \Psi^{(n)}\right)\left(\left(\frac{\left(x_{n}+c_{n}\right) \nu_{n-1}+\eta_{n-1}}{\mu_{n-1}} \circ \Psi^{(n)}\right) \tilde{\kappa}_{1}^{n-1}+\tilde{\kappa}_{2}^{n-1}\right)+\left(\left(\mathrm{L}_{\kappa_{2}^{n}} \Phi_{n}\right) \circ \Psi^{(n)}\right) \tilde{\kappa}_{1}^{n} .
\end{aligned}
$$

By Proposition 7.2, we know that there exist two smooth functions $\mu_{n}$ and $\eta_{n}$ (with $\mu_{n} \neq 0$ ) such that

$$
\Phi_{*}^{(n)}\left(\kappa_{2}^{n}\right)=\left(\eta_{n} \circ \Psi^{(n)}\right) \tilde{\kappa}_{1}^{n}+\left(\mu_{n} \circ \Psi^{(n)}\right) \tilde{\kappa}_{2}^{n} .
$$

Comparing the last two relations and taking into account that $\tilde{\kappa}_{1}^{n}=\frac{\partial}{\partial \tilde{x}_{n}}$ while $\tilde{\kappa}_{1}^{n-1}, \tilde{\kappa}_{2}^{n-1}$, and $\tilde{\kappa}_{2}^{n}$ have zeros as components multiplying $\frac{\partial}{\partial \tilde{x}_{n}}$ we see that $\eta_{n}=\mathrm{L}_{\kappa_{2}^{n}} \Phi_{n}$. From the inductive definition of Kumpera-Ruiz normal forms (regular prolongation) given in Section 2, we have

$$
\tilde{\kappa}_{2}^{n}=\left(\tilde{x}_{n}+\tilde{c}_{n}\right) \tilde{\kappa}_{1}^{n-1}+\tilde{\kappa}_{2}^{n-1} .
$$


We can now conclude that $\mu_{n}=\mu_{n-1}$ and that

$$
\Phi_{n}(x)=\frac{1}{\mu_{n-1}}\left(\left(x_{n}+c_{n}\right) \nu_{n-1}+\eta_{n-1}\right)-\tilde{c}_{n},
$$

where

$$
\tilde{c}_{n}=c_{n} \frac{\nu_{n-1}(0)}{\mu_{n-1}(0)}+\frac{\eta_{n-1}(0)}{\mu_{n-1}(0)}
$$

Now consider $\kappa_{1}^{n}$. Relation (36) gives $\Phi_{*}^{(n)}\left(\kappa_{1}^{n}\right)=\left(\left(\mathrm{L}_{\kappa_{1}^{n}} \Phi_{n}\right) \circ \Psi^{(n)}\right) \tilde{\kappa}_{1}^{n}$, which implies $\nu_{n}=\mathrm{L}_{\kappa_{1}^{n}} \Phi_{n}$. This obviously gives $\nu_{n}=\nu_{i-1} / \mu_{i-1}$.

Singular case: If $w_{n-4}=a_{1}$ then we have $\kappa_{2}^{n}=\kappa_{1}^{n-1}+x_{n} \kappa_{2}^{n-1}$. Together with relation (36) and with the induction hypothesis, this relation leads to:

$$
\begin{aligned}
\Phi_{*}^{(n)}\left(\kappa_{2}^{n}\right)= & \Phi_{*}^{(n-1)}\left(\kappa_{1}^{n-1}\right)+\left(x_{n} \circ \Psi^{(n)}\right) \Phi_{*}^{(n-1)}\left(\kappa_{2}^{n-1}\right) \\
& +\left(\left(\mathrm{L}_{\kappa_{2}^{n}} \Phi_{n}\right) \circ \Psi^{(n)}\right) \tilde{\kappa}_{1}^{n} \\
= & \left(\left(\nu_{n-1}+x_{n} \eta_{n-1}\right) \circ \Psi^{(n)}\right) \tilde{\kappa}_{1}^{n-1}+\left(x_{n} \mu_{n-1} \circ \Psi^{(n)}\right) \tilde{\kappa}_{2}^{n-1} \\
& +\left(\left(\mathrm{L}_{\kappa_{2}^{n}} \Phi_{n}\right) \circ \Psi^{(n)}\right) \tilde{\kappa}_{1}^{n} \\
= & \left(\left(\nu_{n-1}+x_{n} \eta_{n-1}\right) \circ \Psi^{(n)}\right)\left(\tilde{\kappa}_{1}^{n-1}+\left(\frac{x_{n} \mu_{n-1}}{\nu_{n-1}+x_{n} \eta_{n-1}} \circ \Psi^{(n)}\right) \tilde{\kappa}_{2}^{n-1}\right) \\
& +\left(\left(\mathrm{L}_{\kappa_{2}^{n}} \Phi_{n}\right) \circ \Psi^{(n)}\right) \tilde{\kappa}_{1}^{n} .
\end{aligned}
$$

By Proposition 7.2, we know that there exist two functions $\mu_{n}$ and $\eta_{n}$ such that

$$
\Phi_{*}^{(n)}\left(\kappa_{2}^{n}\right)=\left(\eta_{n} \circ \Psi^{(n)}\right) \tilde{\kappa}_{1}^{n}+\left(\mu_{n} \circ \Psi^{(n)}\right) \tilde{\kappa}_{2}^{n} .
$$

The same argument as in the regular case implies $\eta_{n}=\mathrm{L}_{\kappa_{2}^{n}} \Phi_{n}, \mu_{n}=\nu_{n-1}+x_{n} \eta_{n-1}$, and

$$
\Phi_{n}(x)=\frac{x_{n} \mu_{n-1}}{\nu_{n-1}+x_{n} \eta_{n-1}} .
$$

Moreover, like in the regular case, the relation

$$
\Phi_{*}^{(n)}\left(\kappa_{1}^{n}\right)=\left(\left(\mathrm{L}_{\kappa_{1}^{n}} \Phi_{n}\right) \circ \Psi^{(n)}\right) \tilde{\kappa}_{1}^{n}
$$

implies $\nu_{n}=\mathrm{L}_{\kappa_{1}^{n}} \Phi_{n}=\left(\mu_{i-1} \nu_{i-1}\right) /\left(\nu_{i-1}+x_{i} \eta_{i-1}\right)^{2}$.

\subsection{Are Goursat structures locally determined by their abnormal curves?}

In this section we will be interested, in the case of Goursat structures, in the following question asked by Jakubczyk: "are nonholonomic distributions determined by their abnormal curves?". Several results have been obtained giving a positive answer to this question: for stable degenerations of Engel structures by Zhitomirskiı̌ [75], for singular contact structures by Jakubczyk and Zhitomirskiı̌ [28], for generic distributions of corank at least equal to three, at typical points, by Montgomery [45]. Recently, Jakubczyk [26] has proved 
that the answer is positive if we consider abnormal curves of the complexified problem, for all distributions with the exception of a small subclass. We will show in this section that this subclass contains Goursat structures.

To start with, let us be more precise on what we mean by the statement that distributions are determined by their abnormal curves. We will follow the definitions given in [45]. Distributions that belong to a class $\mathcal{Q}$ of distributions are strongly determined by their abnormal curves if, for any pair of distributions $\mathcal{D}$ and $\tilde{\mathcal{D}}$ that belong to $\mathcal{Q}$, any local diffeomorphism that transforms each abnormal curve of $\mathcal{D}$ into an abnormal curve of $\tilde{\mathcal{D}}$, and the other way around, transforms also $\mathcal{D}$ into $\tilde{\mathcal{D}}$. It is clear that Goursat structures are not strongly determined by their abnormal curves because they have very few abnormal curves. For example, contact structures do not have any non-trivial abnormal curve.

A weaker property can be defined as follows. Distributions that belong to a class $\mathcal{Q}$ of distributions are weakly determined by their abnormal curves if, for any pair of distributions $\mathcal{D}$ and $\tilde{\mathcal{D}}$ that belong to $\mathcal{Q}$, the existence of a local diffeomorphism that transforms each abnormal curve of $\mathcal{D}$ into an abnormal curve of $\tilde{\mathcal{D}}$, and the other way around, implies the local equivalence of $\mathcal{D}$ and $\tilde{\mathcal{D}}$.

Proposition 7.4. Goursat structures on n-manifolds are not weakly determined by their abnormal curves if $n \geq 6$.

Proof of Proposition 7.4. Consider the two following Kumpera-Ruiz normal forms defined on $\mathbb{R}^{6}$ by

$$
\left(\frac{\partial}{\partial x_{6}}, x_{6} \frac{\partial}{\partial x_{5}}+x_{5} \frac{\partial}{\partial x_{4}}+x_{4} \frac{\partial}{\partial x_{3}}+x_{3} \frac{\partial}{\partial x_{2}}+\frac{\partial}{\partial x_{1}}\right)
$$

and

$$
\left(\frac{\partial}{\partial x_{6}},\left(x_{6}+1\right) \frac{\partial}{\partial x_{5}}+\frac{\partial}{\partial x_{4}}+x_{5}\left(x_{4} \frac{\partial}{\partial x_{3}}+x_{3} \frac{\partial}{\partial x_{2}}+\frac{\partial}{\partial x_{1}}\right)\right) .
$$

On the one hand, by Theorem 6.2, the distributions spanned by these two Kumpera-Ruiz normal forms have the same abnormal curves, locally at zero. Indeed, for each of them, the submanifolds $S_{j}^{(j)}$, for $j=0$ and 1 , are empty in a small enough neighborhood of zero (see Prop. 4.4); and thus their abnormal curves are given, in both cases, by $\mathcal{A}^{(0)}=\mathcal{C}_{0}=\left(\frac{\partial}{\partial x_{6}}\right)$, in a small enough neighborhood of zero. But on the other hand, it has been shown by Kumpera and Ruiz [32] that these two distributions are not locally equivalent at zero. Indeed, the first one has singularity type $a_{0} a_{0} a_{0}$ at zero while the second one has singularity type $a_{0} a_{1} a_{0}$ at zero. Analogous examples can be constructed for any $n \geq 6$.

Our study of relations between abnormal curves and their singularity type shows that the geometry of a Goursat structure is reflected by abnormal curves of all elements of the derived flag. It is thus natural to introduce the following definition. Distributions that belong to a class $\mathcal{Q}$ of distributions are weakly determined by abnormal curves of their derived flags if, for any pair of distributions $\mathcal{D}$ and $\tilde{\mathcal{D}}$ that belong to $\mathcal{Q}$, the existence of a local diffeomorphism that transforms each abnormal curve of $\mathcal{D}^{(i)}$ into an abnormal curve of $\tilde{\mathcal{D}}^{(i)}$, and the other way around, for each $i \geq 0$, implies the local equivalence of $\mathcal{D}$ and $\tilde{\mathcal{D}}$. It is a direct consequence of Theorem 6.2 and of the classification obtained in [12,19], and [32], that Goursat structures on $\mathbb{R}^{n}$, for $3 \leq n \leq 8$ are determined by abnormal curves of their derived flags. It is surprising that in higher dimensions it is not the case. Indeed, we have the following result which is a direct consequence of Theorem 6.2 and the theorem announced in [13].

Proposition 7.5. Goursat structures on n-manifolds are not weakly determined by abnormal curves of their derived flags if $n \geq 9$.

It has already been announced in [13] that the growth vector is not a complete invariant for Goursat structures on $\mathbb{R}^{n}$, for $n \geq 9$ (which, together with Th. 6.2 , implies the above result). We will give in this section our proof of this latter fact. An alternative proof, together with a complete classification, can be found in [50]. 
It is important to stress that the method used in [50] and the method that we will present in this section are different. It seems that both methods apply, in general, to different cases of non-equivalence.

Our aim now is to prove Proposition 7.5. This will be done by giving an example (Prop. 7.6) of two Goursat structures $\mathcal{D}$ and $\tilde{\mathcal{D}}$ that are locally non-equivalent but that have the same singularity type (which, by Th. 6.2, implies the existence of a diffeomorphism between the abnormal curves of $\mathcal{D}^{(i)}$ and those of $\tilde{\mathcal{D}}^{(i)}$, for $\left.i \geq 0\right)$. Then, this example will be improved (Prop. 7.7) by constructing, instead of a pair of distributions, a continuous family (parametrized by a real number) of locally non-equivalent Goursat structures that have the same singularity type, and thus diffeomorphic collections of abnormal curves for all elements of their derived flags.

Consider two Kumpera-Ruiz normal forms $\left(\kappa_{1}^{n}, \kappa_{2}^{n}\right)$ and $\left(\tilde{\kappa}_{1}^{n}, \tilde{\kappa}_{2}^{n}\right)$, defined on $\mathbb{R}^{n}$, centered at zero, and given, respectively, in coordinates $x=\left(x_{1}, \ldots, x_{n}\right)$ and $\tilde{x}=\left(\tilde{x}_{1}, \ldots, \tilde{x}_{n}\right)$. Assume that they have been obtained from $\left(\kappa_{1}^{i}, \kappa_{2}^{i}\right)$ and $\left(\tilde{\kappa}_{1}^{i}, \tilde{\kappa}_{2}^{i}\right)$, respectively, by a sequence of regular prolongations, for $i \geq 3$. Suppose, moreover, that the Goursat structures spanned by $\left(\kappa_{1}^{n}, \kappa_{2}^{n}\right)$ and $\left(\tilde{\kappa}_{1}^{n}, \tilde{\kappa}_{2}^{n}\right)$ are locally equivalent and let $\tilde{x}=\Phi(x)$ be a (generalized) contact transformation, of order $n-2$, that establishes this equivalence. We have $\tilde{x}_{j}=\Phi_{j}(x)$, for $1 \leq j \leq n$. We are going to prove that the components $\Phi_{j}$, for $i+1 \leq j \leq n$, can be obtained by a sequence of derivations (with respect to a well chosen vector field) from the component $\Phi_{i}$. To start with, apply Theorem 7.3 to the component $\Phi_{i+1}$. We have

$$
\Phi_{i+1}=\frac{1}{\mu_{i}}\left(\left(x_{i+1}+c_{i+1}\right) \nu_{i}+\eta_{i}\right)-\tilde{c}_{i+1} .
$$

In follows also from Theorem 7.3 (regular case) that this expression can be written in the following form:

$$
\begin{aligned}
\Phi_{i+1} & =\frac{1}{\mu_{i}}\left(\left(x_{i+1}+c_{i+1}\right) \mathrm{L}_{\kappa_{1}^{i}} \Phi_{i}+\mathrm{L}_{\kappa_{2}^{i}} \Phi_{i}\right)-\tilde{c}_{i+1} \\
& =\frac{1}{\mu_{i}} \mathrm{~L}_{\left(x_{i+1}+c_{i+1}\right) \kappa_{1}^{i}+\kappa_{2}^{i}} \Phi_{i}-\tilde{c}_{i+1} \\
& =\mathrm{L}_{\frac{1}{\mu_{i}} \kappa_{2}^{i+1}} \Phi_{i}-\tilde{c}_{i+1} .
\end{aligned}
$$

But since $\Phi_{i}$ is a function of $x_{1}, \ldots, x_{i}$ only, the latter expression can be rewritten as

$$
\Phi_{i+1}=\mathrm{L}_{\frac{1}{\mu_{i}} \kappa_{2}^{n}} \Phi_{i}-\tilde{c}_{i+1} .
$$

Theorem 7.3 implies, moreover, that $\mu_{j}=\mu_{i}$, for $i+1 \leq j \leq n$. Thus, the previous argument can be repeated to obtain, for $1 \leq k \leq n-i$, the following relations:

$$
\Phi_{i+k}=\mathrm{L}_{\frac{1}{\mu_{i}} \kappa_{2}^{n}}^{k} \Phi_{i}-\tilde{c}_{i+k},
$$

which imply that

$$
\tilde{c}_{i+k}=\left(\mathrm{L}_{\frac{1}{\mu_{i}} \kappa_{2}^{n}}^{k} \Phi_{i}\right)(0)
$$

because the coordinates are centered. Therefore, in the case of a sequence of regular prolongations, the constants $\tilde{c}_{i+k}$ can be obtained by computing the successive derivatives $\mathrm{L}_{\left(1 / \mu_{i}\right) \kappa_{2}^{n}}^{k} \Phi_{i}$ of the component $\Phi_{i}$ (that defines the coordinate $\tilde{x}_{i}$ ) and by taking their values at zero.

The following definition is natural and will simplify the proofs of next results given in this section. Let $\gamma$ be a smooth function defined on $\mathbb{R}^{n}$ and let $g$ be a smooth vector field, also defined on $\mathbb{R}^{n}$. The degree of the 
function $\gamma$, with respect to the vector field $g$, is the smallest integer $k$ (maybe infinite) such that $\mathrm{L}_{g}^{k}(\gamma)(0) \neq 0$. Note that if the degree of $\gamma_{1}$ is $i_{1}$ and the degree of $\gamma_{2}$ is $i_{2}$ then the degree of $\gamma_{1} \gamma_{2}$ is obviously $i_{1}+i_{2}$.

Proposition 7.6. Consider the two following Kumpera-Ruiz normal forms defined on $\mathbb{R}^{9}$ by

$$
\begin{aligned}
\kappa_{1}^{9} & =\frac{\partial}{\partial x_{9}} \\
\kappa_{2}^{9}\left(c_{9}\right) & =\left(x_{9}+c_{9}\right) \frac{\partial}{\partial x_{8}}+\left(x_{8}+1\right) \frac{\partial}{\partial x_{7}}+x_{7} \frac{\partial}{\partial x_{6}}+\frac{\partial}{\partial x_{5}}+x_{6}\left(x_{5} \frac{\partial}{\partial x_{4}}+x_{4} \frac{\partial}{\partial x_{3}}+x_{3} \frac{\partial}{\partial x_{2}}+\frac{\partial}{\partial x_{1}}\right),
\end{aligned}
$$

where $c_{9}=0$ or 1 . They are locally non-equivalent at zero, although both of them have the same singularity type $a_{0} a_{0} a_{1} a_{2} a_{0} a_{0}$ at zero.

Proof of Proposition 7.6. Denote by $\kappa^{9}$ the Kumpera-Ruiz normal form given by $\left(\kappa_{1}^{9}, \kappa_{2}^{9}(0)\right)$, in $\left(x_{1}, \ldots, x_{9}\right)$ coordinates, and denote by $\tilde{\kappa}^{9}$ the Kumpera-Ruiz normal form given by $\left(\kappa_{1}^{9}, \kappa_{2}^{9}\left(\tilde{c}_{9}\right)\right)$, in $\left(\tilde{x}_{1}, \ldots, \tilde{x}_{9}\right)$-coordinates. We are going to show that if a (generalized) contact transformation $\tilde{x}=\Phi(x)$ converts the Goursat structure generated by $\kappa^{9}$ into the one generated by $\tilde{\kappa}^{9}$ then we must have $\tilde{c}_{9}=0$.

Denote by $\kappa^{4}, \ldots, \kappa^{9}$ and by $\tilde{\kappa}^{4}, \ldots, \tilde{\kappa}^{9}$ the elements of the two sequences of Kumpera-Ruiz normal forms used to construct, via prolongations, the normal forms $\kappa^{9}$ and $\tilde{\kappa}^{9}$, respectively. Since $\kappa^{5}=R_{0}\left(\kappa^{4}\right)$ we have, by the regular case of Theorem 7.3, the following relations:

$$
\mu_{5}=\mu_{4} \quad \text { and } \quad \nu_{5}=\frac{\nu_{4}}{\mu_{4}} .
$$

Hence $\mu_{5}$ and $\nu_{5}$ are functions of $x_{1}, \ldots, x_{4}$ only. Denote $\mu=\mu_{4}, \nu=\nu_{4}$, and $\eta=\eta_{5}$. Since $\kappa^{6}=S\left(\kappa^{5}\right)$ we have, by the singular case of Theorem 7.3, the following relations:

$$
\begin{aligned}
\Phi_{6}(x) & =\frac{x_{6} \mu}{\frac{\nu}{\mu}+x_{6} \eta} \\
\mu_{6} & =\frac{\nu}{\mu}+x_{6} \eta .
\end{aligned}
$$

Denote $\alpha=1 / \mu_{6}$ and $g=\alpha \kappa_{2}^{9}$. Since both $\kappa^{9}$ and $\tilde{\kappa}^{9}$ are obtained by a sequence of regular prolongations from $\kappa^{6}$ and $\tilde{\kappa}^{6}$, respectively, it follows from the discussion given at the beginning of this section that the new constant $\tilde{c}_{9}$ can be calculated by computing the successive derivatives of $\Phi_{6}$, in the direction of the vector field $g=\left(1 / \mu_{6}\right) \kappa_{2}^{9}$. Namely

$$
\tilde{c}_{9}=\left(\mathrm{L}_{g}^{3} \Phi_{6}\right)(0)
$$

Instead of computing the successive derivatives of $\Phi_{6}$ directly, take the Taylor series expansion of $\Phi_{6}$. The terms of this expansion that contain coordinate functions of degree $d \geq 4$, with respect to $g$, can obviously be discarded. To this aim, we will start by computing the degree, with respect to $g$, of the functions $x_{1}, \ldots, x_{6}$, that is of the variables on which $\Phi_{6}$ depends.

For $x_{6}$, we have:

$$
\begin{aligned}
\mathrm{L}_{g} x_{6} & =\alpha x_{7} \\
\mathrm{~L}_{g}^{2} x_{6} & =\alpha^{2}\left(x_{8}+1\right)+\left(\mathrm{L}_{g} \alpha\right) x_{7} \\
\mathrm{~L}_{g}^{3} x_{6} & =\alpha^{3} x_{9}+3 \alpha\left(\mathrm{L}_{g} \alpha\right)\left(x_{8}+1\right)+\left(\mathrm{L}_{g}^{2} \alpha\right) x_{7} .
\end{aligned}
$$


Since $\left(\mathrm{L}_{g}^{2} x_{6}\right)(0)=\alpha^{2}(0)=(\mu(0) / \nu(0))^{2} \neq 0$, the degree of $x_{6}$ is 2 . We have $\mathrm{L}_{g} x_{5}=\alpha$. Therefore the degree of $x_{5}$ is 1 . We have $\mathrm{L}_{g} x_{4}=\alpha x_{6} x_{5}$. Thus the degree of $x_{4}$ is 4 . Analogously, the degree of $x_{3}$ is 7 , the degree of $x_{2}$ is 10 , and the degree of $x_{1}$ is 3 .

Now observe that $\Phi_{6}(x)=x_{6} \varphi\left(x_{1}, \ldots, x_{6}\right)$, for a suitable function $\varphi$. This implies that each term of the Taylor series expansion of $\Phi_{6}$ is of the form $x_{6} x_{1}^{k_{1}} \cdots x_{6}^{k_{6}}$, for some integers $k_{1}, \ldots, k_{6}$. Since $\tilde{c}_{9}=\left(\mathrm{L}_{g}^{3} \Phi_{6}\right)(0)$, we consider only terms of degree $d \leq 3$ with respect to $g$. Therefore we have:

$$
\Phi_{6}(x)=A x_{6}+B x_{6} x_{5}
$$

up to terms of degree $d \geq 4$ with respect to $g$. Recall that neither $\mu$ nor $\nu$ depend on the variables $x_{5}$ and $x_{6}$. Hence

$$
\begin{aligned}
\frac{\partial \Phi_{6}}{\partial x_{6}} & =\frac{\nu}{\left(\frac{\nu}{\mu}+x_{6} \eta\right)^{2}} \\
\frac{\partial^{2} \Phi_{6}}{\partial x_{5} \partial x_{6}} & =\frac{-2 x_{6} \eta_{x_{5}}}{\left(\frac{\nu}{\mu}+x_{6} \eta\right)^{3}}
\end{aligned}
$$

Thus $A=\mu(0)$ and $B=0$. This implies that $\Phi_{6}(x)=\mu(0) x_{6}$, up to terms of degree $d \geq 4$. Since we have already computed the successive derivatives of $x_{6}$, it is easy to obtain that:

$$
\begin{aligned}
& \left(\mathrm{L}_{g} \Phi_{6}\right)(0)=0 \\
& \left(\mathrm{~L}_{g}^{2} \Phi_{6}\right)(0)=\mu(0) \alpha^{2}(0) \\
& \left(\mathrm{L}_{g}^{3} \Phi_{6}\right)(0)=3 \mu(0) \alpha(0)\left(\mathrm{L}_{g} \alpha\right)(0)
\end{aligned}
$$

But $\left(\mathrm{L}_{g} \alpha\right)(0)=0$. Hence, since $\tilde{c}_{9}=\left(\mathrm{L}_{g}^{3} \Phi_{6}\right)(0)$, we have $\tilde{c}_{9}=0$.

Proposition 7.7. Consider the following family of Kumpera-Ruiz normal forms defined on $\mathbb{R}^{11}$ by

$$
\begin{aligned}
\kappa_{1}^{11}= & \frac{\partial}{\partial x_{11}} \\
\kappa_{2}^{11}\left(c_{11}\right)= & \left(x_{11}+c_{11}\right) \frac{\partial}{\partial x_{10}}+\left(x_{10}+1\right) \frac{\partial}{\partial x_{9}}+\left(x_{9}+1\right) \frac{\partial}{\partial x_{8}}+x_{8} \frac{\partial}{\partial x_{7}} \\
& +x_{7} \frac{\partial}{\partial x_{6}}+\frac{\partial}{\partial x_{5}}+x_{6}\left(x_{5} \frac{\partial}{\partial x_{4}}+x_{4} \frac{\partial}{\partial x_{3}}+x_{3} \frac{\partial}{\partial x_{2}}+\frac{\partial}{\partial x_{1}}\right)
\end{aligned}
$$

where $c_{11}$ is an arbitrary real constant. Two Kumpera-Ruiz normal forms that belong to this family are locally equivalent at zero if and only if they have the same constant $c_{11}$, although all of them have the same singularity type $a_{0} a_{0} a_{1} a_{2} a_{3} a_{0} a_{0} a_{0}$ at zero.

Proof of Proposition 7.7. Denote by $\kappa^{11}$ the Kumpera-Ruiz normal form given by $\left(\kappa_{1}^{11}, \kappa_{2}^{11}\left(c_{11}\right)\right)$, in coordinates $\left(x_{1}, \ldots, x_{11}\right)$, and denote by $\tilde{\kappa}^{11}$ the Kumpera-Ruiz normal form given by $\left(\kappa_{1}^{11}, \kappa_{2}^{11}\left(\tilde{c}_{11}\right)\right)$, in coordinates $\left(\tilde{x}_{1}, \ldots, \tilde{x}_{11}\right)$. We are going to show that if a (generalized) contact transformation $\tilde{x}=\Phi(x)$ converts the Goursat structure generated by $\kappa^{11}$ into the one generated by $\tilde{\kappa}^{11}$ then we must have $\tilde{c}_{11}=c_{11}$.

Denote by $\kappa^{4}, \ldots, \kappa^{11}$ and by $\tilde{\kappa}^{4}, \ldots, \tilde{\kappa}^{11}$ the elements of the two sequences of Kumpera-Ruiz normal forms used to construct, via prolongations, the normal forms $\kappa^{11}$ and $\tilde{\kappa}^{11}$, respectively. Since $\kappa^{5}=R_{0}\left(\kappa^{4}\right)$ we have, by the regular case of Theorem 7.3 , the following relations:

$$
\mu_{5}=\mu_{4} \quad \text { and } \quad \nu_{5}=\frac{\nu_{4}}{\mu_{4}} .
$$


Hence $\mu_{5}$ and $\nu_{5}$ are functions of $x_{1}, \ldots, x_{4}$ only. Denote $\mu=\mu_{4}, \nu=\nu_{4}$, and $\eta=\eta_{5}$. Since $\kappa^{6}=S\left(\kappa^{5}\right)$ we have, by the singular case of Theorem 7.3 , the following relations:

$$
\begin{aligned}
\Phi_{6}(x) & =\frac{x_{6} \mu}{\frac{\nu}{\mu}+x_{6} \eta} \\
\mu_{6} & =\frac{\nu}{\mu}+x_{6} \eta .
\end{aligned}
$$

Denote $\alpha=1 / \mu_{6}$ and $g=\alpha \kappa_{2}^{11}$. Since both $\kappa^{11}$ and $\tilde{\kappa}^{11}$ are obtained by a sequence of regular prolongations from $\kappa^{6}$ and $\tilde{\kappa}^{6}$, respectively, it follows from the discussion given at the beginning of this section that the new constant $\tilde{c}_{11}$ can be obtained by computing the successive derivatives of $\Phi_{6}$, in the direction of the vector field $g$. Namely

$$
\tilde{c}_{11}=\left(\mathrm{L}_{g}^{5} \Phi_{6}\right)(0)
$$

Let us consider the Taylor series expansion of $\Phi_{6}$. Again, the terms of this expansion that contain coordinate functions of degree $d \geq 6$, with respect to $g$, will be discarded. The successive derivatives of $x_{6}$ are given by:

$$
\begin{aligned}
\mathrm{L}_{g} x_{6}= & \alpha x_{7} \\
\mathrm{~L}_{g}^{2} x_{6}= & \alpha^{2} x_{8}+\left(\mathrm{L}_{g} \alpha\right) x_{7} \\
\mathrm{~L}_{g}^{3} x_{6}= & \alpha^{3}\left(x_{9}+1\right)+3 \alpha\left(\mathrm{L}_{g} \alpha\right) x_{8}+\left(\mathrm{L}_{g}^{2} \alpha\right) x_{7} \\
\mathrm{~L}_{g}^{4} x_{6}= & \alpha^{4}\left(x_{10}+1\right)+6 \alpha^{2}\left(\mathrm{~L}_{g} \alpha\right)\left(x_{9}+1\right)+\left(3\left(\mathrm{~L}_{g} \alpha\right)^{2}+4 \alpha\left(\mathrm{L}_{g}^{2} \alpha\right)\right) x_{8}+\left(\mathrm{L}_{g}^{3} \alpha\right) x_{7} \\
\mathrm{~L}_{g}^{5} x_{6}= & \alpha^{5}\left(x_{11}+c_{11}\right)+10 \alpha^{3}\left(\mathrm{~L}_{g} \alpha\right)\left(x_{10}+1\right)+\left(15 \alpha\left(\mathrm{L}_{g} \alpha\right)^{2}+10 \alpha^{2}\left(\mathrm{~L}_{g}^{2} \alpha\right)\right)\left(x_{9}+1\right) \\
& +\left(10\left(\mathrm{~L}_{g} \alpha\right)\left(\mathrm{L}_{g}^{2} \alpha\right)+5 \alpha\left(\mathrm{L}_{g}^{3} \alpha\right)\right) x_{8}+\left(\mathrm{L}_{g}^{4} \alpha\right) x_{7} .
\end{aligned}
$$

Since $\left(\mathrm{L}_{g}^{3} x_{6}\right)(0)=\alpha^{3}(0)=(\mu(0) / \nu(0))^{3} \neq 0$, the degree of $x_{6}$ is 3 . The degree of $x_{5}$ is 1 , the degree of $x_{4}$ is 5 , the degree of $x_{3}$ is 9 , the degree of $x_{2}$ is 13 , and the degree of $x_{1}$ is 4 (all degrees are with respect to $g$ ).

Now observe that $\Phi_{6}(x)=x_{6} \varphi\left(x_{1}, \ldots, x_{6}\right)$, for a suitable function $\varphi$. The Taylor series expansion of $\Phi_{6}$, up to terms of degree $d \leq 5$ with respect to $g$, is given by:

$$
\Phi_{6}(x)=A x_{6}+B x_{6} x_{5}+C x_{6} x_{5}^{2} .
$$

Recall that neither $\mu$ nor $\nu$ depend on the variable $x_{5}$. Therefore

$$
\begin{aligned}
\frac{\partial \Phi_{6}}{\partial x_{6}}(0) & =\mu(0) \\
\frac{\partial^{2} \Phi_{6}}{\partial x_{5} \partial x_{6}}(0) & =\frac{\partial^{3} \Phi_{6}}{\partial^{2} x_{5} \partial x_{6}}(0)=0 .
\end{aligned}
$$

Hence $A=\mu(0)$ and both $B$ and $C$ are equal to 0 . This implies that $\Phi_{6}(x)=\mu(0) x_{6}$, up to terms of degree $d \geq 6$. Since we have already computed the successive derivatives of $x_{6}$, it is easy to obtain that:

$$
\begin{aligned}
& \left(\mathrm{L}_{g} \Phi_{6}\right)(0)=0 \\
& \left(\mathrm{~L}_{g}^{2} \Phi_{6}\right)(0)=0 \\
& \left(\mathrm{~L}_{g}^{3} \Phi_{6}\right)(0)=\mu(0) \alpha^{3}(0) \\
& \left(\mathrm{L}_{g}^{4} \Phi_{6}\right)(0)=\mu(0) \alpha^{4}(0) \\
& \left(\mathrm{L}_{g}^{5} \Phi_{6}\right)(0)=\mu(0) \alpha^{5}(0) c_{11} .
\end{aligned}
$$


Since $\Phi$ transforms $\kappa^{11}$ into $\tilde{\kappa}^{11}$, we must have both $\tilde{c}_{9}=1$ and $\tilde{c}_{10}=1$. But $\tilde{c}_{9}=\left(\mathrm{L}_{g}^{3} \Phi_{6}\right)(0)$ and $\tilde{c}_{10}=$ $\left(\mathrm{L}_{g}^{4} \Phi_{6}\right)(0)$. Therefore, $\mu^{4}(0) / \nu^{3}(0)=1$ and $\mu^{5}(0) / \nu^{4}(0)=1$. This obviously implies $\mu(0)=\nu(0)=1$. Hence, since $\tilde{c}_{11}=c_{11} \mu^{6}(0) / \nu^{5}(0)$, we have $\tilde{c}_{11}=c_{11}$.

\section{Appendix A. Proof of Kumpera-Ruiz theorem}

Our proof of Theorem 2.3 will be based on the following classical result, which was originally formulated in the dual language of Pfaffian systems ([72], Th. V) (see also [11,22,32], and Appendix C).

Proposition A.1 (von Weber). Let $\mathcal{D}$ be a Goursat structure on a manifold $M$ of dimension $n \geq 4$. In a small enough neighborhood of any point $p$ in $M$, the distribution $\mathcal{D}$ is equivalent to a distribution spanned on $\mathbb{R}^{n}$ by a pair of vector fields that has the following form:

$$
\left(\frac{\partial}{\partial y_{n}}, y_{n} \zeta_{1}^{n-1}+\zeta_{2}^{n-1}\right)
$$

where $\zeta_{1}^{n-1}$ and $\zeta_{2}^{n-1}$ are the lifts of a pair of vector fields that span a Goursat structure on $\mathbb{R}^{n-1}$ and the coordinates $y_{1}, \ldots, y_{n}$ are centered at $p$.

Proof of Proposition A.1. It is well known (see e.g. [7,67], and [77]) that any Goursat structure $\mathcal{D}$ on a manifold of dimension $n \geq 4$ admits a canonical line field $\mathcal{L} \subset \mathcal{D}$ uniquely defined by $\left[\mathcal{L}, \mathcal{D}^{(1)}\right] \subset \mathcal{D}^{(1)}$. Observe that in the preliminary normal form $(37)$ of Proposition A.1 this line field is given by $\mathcal{L}=\left(\frac{\partial}{\partial y_{n}}\right)$.

It is clear that, applying around $p$ the flow-box theorem to a vector field that spans $\mathcal{L}$, we can chose local coordinates $\left(z_{1}, \ldots, z_{n}\right)$, centered at $p$, such that $\mathcal{D}$ is locally equivalent to a distribution spanned on $\mathbb{R}^{n}$ by a pair of vector fields that has the following form:

$$
\left(\frac{\partial}{\partial z_{n}}, \sum_{i=2}^{n-1} \alpha_{i}(z) \frac{\partial}{\partial z_{i}}+\frac{\partial}{\partial z_{1}}\right)
$$

where $\mathcal{L}=\left(\frac{\partial}{\partial z_{n}}\right)$. Since $\operatorname{dim} \mathcal{D}^{(1)}(p)=3$ there exists an integer $i$ such that $\frac{\partial \alpha_{i}}{\partial z_{n}}(p) \neq 0$. We can assume that $i=n-1$ and, moreover, that $\alpha_{n-1}(0)=0$. Otherwise, replace the coordinate $z_{n-1}$ by $z_{n-1}-z_{1} \alpha_{n-1}(0)$. Now, if we define $y_{n}=\alpha_{n-1}(z)$ and $y_{i}=z_{i}$, for $1 \leq i \leq n-1$, we get that $\mathcal{D}$ is locally equivalent to a distribution spanned on $\mathbb{R}^{n}$ by a pair of vector fields that has the following form:

$$
\left(\frac{\partial}{\partial y_{n}}, y_{n} \frac{\partial}{\partial y_{n-1}}+\sum_{i=2}^{n-2} \beta_{i}(y) \frac{\partial}{\partial y_{i}}+\frac{\partial}{\partial y_{1}}\right) .
$$

But the inclusion $\left[\mathcal{L}, \mathcal{D}^{(1)}\right] \subset \mathcal{D}^{(1)}$ clearly implies $\frac{\partial^{2} \beta_{i}}{\partial y_{n}^{2}} \equiv 0$ for $2 \leq i \leq n-2$. That is $\beta_{i}(y)=a_{i}\left(\bar{y}_{n-1}\right) y_{n}+$ $b_{i}\left(\bar{y}_{n-1}\right)$, where $\bar{y}_{n-1}=\left(y_{1}, \ldots, y_{n-1}\right)$. Define

$$
\zeta_{1}^{n-1}=\frac{\partial}{\partial y_{n-1}}+\sum_{i=2}^{n-2} a_{i}\left(\bar{y}_{n-1}\right) \frac{\partial}{\partial y_{i}} \quad \text { and } \quad \zeta_{2}^{n-2}=\sum_{i=2}^{n-2} b_{i}\left(\bar{y}_{n-1}\right) \frac{\partial}{\partial y_{i}}+\frac{\partial}{\partial y_{1}} .
$$

We conclude that $\mathcal{D}$ is equivalent to $\left(\frac{\partial}{\partial y_{n}}, y_{n} \zeta_{1}^{n-1}+\zeta_{2}^{n-1}\right)$, where both $\zeta_{1}^{n-1}$ and $\zeta_{2}^{n-1}$ are lifts (see Notation 2.1) of vector fields defined on $\mathbb{R}^{n-1}$. Put $\mathcal{F}=\left(\zeta_{1}^{n-1}, \zeta_{2}^{n-1}\right)$. Clearly $\operatorname{dim} \mathcal{D}^{(i+1)}=\operatorname{dim} \mathcal{F}^{(i)}+1$, for $0 \leq i \leq n-3$. It follows that the distribution $\mathcal{F}$ is a Goursat structure on $\mathbb{R}^{n-1}$. 
Proof of Theorem 2.3. On three-manifolds, Theorem 2.3 is obviously true. Indeed, it is the solution of the Pfaff problem (see [15] and [18]; see also [6] for a modern approach). We will proceed by induction on $n \geq 4$, showing that if any Goursat structure on an $(n-1)$-manifold can be converted locally into a Kumpera-Ruiz normal form then the same is true for any Goursat structure on a manifold of dimension $n$.

Let $\mathcal{D}$ be a Goursat structure on a manifold $M$ of dimension $n \geq 4$ and let $p$ be an arbitrary point in $M$. It follows from Proposition A.1 that $\mathcal{D}$ is equivalent, in a small enough neighborhood of $p$, to a distribution spanned on $\mathbb{R}^{n}$ by a pair of vector fields $\left(\zeta_{1}^{n}, \zeta_{2}^{n}\right)$ that has the following form:

$$
\begin{aligned}
\zeta_{1}^{n} & =\frac{\partial}{\partial y_{n}} \\
\zeta_{2}^{n} & =y_{n} \zeta_{1}^{n-1}+\zeta_{2}^{n-1} .
\end{aligned}
$$

In the rest of the proof we will assume that $\mathcal{D}=\left(\zeta_{1}^{n}, \zeta_{2}^{n}\right)$. Note that the $y$-coordinates are centered at zero.

Our aim is to build a local change of coordinates

$$
\left(x_{1}, \ldots, x_{n}\right)=\phi^{n}\left(y_{1}, \ldots, y_{n}\right),
$$

a Kumpera-Ruiz normal form $\left(\kappa_{1}^{n}, \kappa_{2}^{n}\right)$ on $\mathbb{R}^{n}$, and three smooth functions $\mu_{n}, \nu_{n}$, and $\eta_{n}$ such that

$$
\begin{aligned}
& \phi_{*}^{n}\left(\zeta_{1}^{n}\right)=\left(\nu_{n} \circ \psi^{n}\right) \kappa_{1}^{n} \\
& \phi_{*}^{n}\left(\zeta_{2}^{n}\right)=\left(\eta_{n} \circ \psi^{n}\right) \kappa_{1}^{n}+\left(\mu_{n} \circ \psi^{n}\right) \kappa_{2}^{n},
\end{aligned}
$$

where $\psi^{n}=\left(\phi^{n}\right)^{-1}$ denotes the inverse of the local diffeomorphism $\phi^{n}$ and both $\mu_{n}(0) \neq 0$ and $\nu_{n}(0) \neq 0$. Moreover, we will impose the $x$-coordinates to be centered at zero. That is $\phi^{n}(0)=0$. Observe that the triangular form in (38) appears because both $\zeta_{1}^{n}$ and $\kappa_{1}^{n}$ span the canonical line fields of the distributions spanned by $\left(\zeta_{1}^{n}, \zeta_{2}^{n}\right)$ and $\left(\kappa_{1}^{n}, \kappa_{2}^{n}\right)$, respectively.

By Proposition A.1, the distribution spanned by $\left(\zeta_{1}^{n}, \zeta_{2}^{n}\right)$ is defined by the lifts of a pair of vector fields $\left(\zeta_{1}^{n-1}, \zeta_{2}^{n-1}\right)$ that span a Goursat structure on $\mathbb{R}^{n-1}$. Since the theorem is assumed to be true on $\mathbb{R}^{n-1}$, the distribution spanned by $\left(\zeta_{1}^{n-1}, \zeta_{2}^{n-1}\right)$ is locally equivalent to a Kumpera-Ruiz normal form $\left(\kappa_{1}^{n-1}, \kappa_{2}^{n-1}\right)$ defined on $\mathbb{R}^{n-1}$ and centered at zero. It follows that there exists a local diffeomorphism $\left(x_{1}, \ldots, x_{n-1}\right)=$ $\phi^{n-1}\left(y_{1}, \ldots, y_{n-1}\right)$ and four smooth functions $\nu_{n-1}, \lambda_{n-1}, \eta_{n-1}$, and $\mu_{n-1}$ such that:

$$
\begin{aligned}
& \phi_{*}^{n-1}\left(\zeta_{1}^{n-1}\right)=\left(\nu_{n-1} \circ \psi^{n-1}\right) \kappa_{1}^{n-1}+\left(\lambda_{n-1} \circ \psi^{n-1}\right) \kappa_{2}^{n-1} \\
& \phi_{*}^{n-1}\left(\zeta_{2}^{n-1}\right)=\left(\eta_{n-1} \circ \psi^{n-1}\right) \kappa_{1}^{n-1}+\left(\mu_{n-1} \circ \psi^{n-1}\right) \kappa_{2}^{n-1},
\end{aligned}
$$

where $\psi^{n-1}=\left(\phi^{n-1}\right)^{-1}$ denotes the inverse of the local diffeomorphism $\phi^{n-1}$ and $\left(\nu_{n-1} \mu_{n-1}-\lambda_{n-1} \eta_{n-1}\right)(0) \neq 0$.

Let $\phi^{n}=\left(\phi^{n-1}, \phi_{n}\right)^{T}$ be a diffeomorphism of $\mathbb{R}^{n}$ such that $\phi^{n-1}$ depends on the first $n-1$ coordinates only. Moreover, let $f$ be a vector field on $\mathbb{R}^{n}$ of the form $f=\alpha f^{n-1}+f_{n}$, where $\alpha$ is a smooth function on $\mathbb{R}^{n}$, the vector field $f^{n-1}$ is the lift of a vector field on $\mathbb{R}^{n-1}$ (see Notation 2.1), and the only non-zero component of $f_{n}$ is the last one. A direct computation shows that:

$$
\phi_{*}^{n}(f)=\left(\alpha \circ \psi^{n}\right) \phi_{*}^{n-1}\left(f^{n-1}\right)+\left(\left(\mathrm{L}_{f} \phi_{n}\right) \circ \psi^{n}\right) \frac{\partial}{\partial x_{n}} .
$$

Note that the vector field $\phi_{*}^{n-1}\left(f^{n-1}\right)$ is lifted along the $x_{n}$-coordinate, which is defined by $\phi_{n}$. 
Regular case: If $\mu_{n-1}(0) \neq 0$ then we can complete $\phi^{n-1}$ to a zero-preserving local diffeomorphism of $\mathbb{R}^{n}$ by taking $\phi^{n}=\left(\phi^{n-1}, \phi_{n}\right)^{T}$, where

$$
\phi_{n}(y)=\frac{\nu_{n-1} y_{n}+\eta_{n-1}}{\lambda_{n-1} y_{n}+\mu_{n-1}}-\frac{\eta_{n-1}(0)}{\mu_{n-1}(0)}
$$

In this case, we define $c_{n}=\left(\eta_{n-1} / \mu_{n-1}\right)(0)$ and

$$
\nu_{n}=\mathrm{L}_{\zeta_{1}^{n}} \phi_{n}, \quad \eta_{n}=\mathrm{L}_{\zeta_{2}^{n}} \phi_{n}, \quad \text { and } \quad \mu_{n}=\lambda_{n-1} y_{n}+\mu_{n-1} .
$$

Observe that $\nu_{n}(0)=\mathrm{L}_{\zeta_{1}^{n}} \phi_{n}(0)=\left(\nu_{n-1} \mu_{n-1}-\lambda_{n-1} \eta_{n-1}\right)(0) \neq 0$ and that $\mu_{n}(0)=\mu_{n-1}(0) \neq 0$. Thus the right hand side of (38) defines a locally invertible transformation. Moreover, the Kumpera-Ruiz normal form $\left(\kappa_{1}^{n}, \kappa_{2}^{n}\right)$ is defined to be the regular prolongation, with parameter $c_{n}$, of $\left(\kappa_{1}^{n-1}, \kappa_{2}^{n-1}\right)$.

Let us check that, in this case, relation (38) holds. Together, relations (39) and (40) give:

$$
\begin{aligned}
\phi_{*}^{n}\left(\zeta_{2}^{n}\right) & =\left(y_{n} \circ \psi^{n}\right) \phi_{*}^{n-1}\left(\zeta_{1}^{n-1}\right)+\phi_{*}^{n-1}\left(\zeta_{2}^{n-1}\right)+\left(\left(\mathrm{L}_{\zeta_{2}^{n}} \phi_{n}\right) \circ \psi^{n}\right) \frac{\partial}{\partial x_{n}} \\
& =\left(\left(\nu_{n-1} y_{n}+\eta_{n-1}\right) \circ \psi^{n}\right) \kappa_{1}^{n-1}+\left(\left(\lambda_{n-1} y_{n}+\mu_{n-1}\right) \circ \psi^{n}\right) \kappa_{2}^{n-1}+\left(\eta_{n} \circ \psi^{n}\right) \kappa_{1}^{n} \\
& =\left(\left(\lambda_{n-1} y_{n}+\mu_{n-1}\right) \circ \psi^{n}\right)\left(\left(\frac{\nu_{n-1} y_{n}+\eta_{n-1}}{\lambda_{n-1} y_{n}+\mu_{n-1}} \circ \psi^{n}\right) \kappa_{1}^{n-1}+\kappa_{2}^{n-1}\right)+\left(\eta_{n} \circ \psi^{n}\right) \kappa_{1}^{n} \\
& =\left(\mu_{n} \circ \psi^{n}\right)\left(\left(x_{n}+c_{n}\right) \kappa_{1}^{n-1}+\kappa_{2}^{n-1}\right)+\left(\eta_{n} \circ \psi^{n}\right) \kappa_{1}^{n} \\
& =\left(\eta_{n} \circ \psi^{n}\right) \kappa_{1}^{n}+\left(\mu_{n} \circ \psi^{n}\right) \kappa_{2}^{n} .
\end{aligned}
$$

Moreover, we have

$$
\phi_{*}^{n}\left(\zeta_{1}^{n}\right)=\left(\left(\mathrm{L}_{\zeta_{1}^{n}} \phi_{n}\right) \circ \psi^{n}\right) \frac{\partial}{\partial x_{n}}=\left(\nu_{n} \circ \psi^{n}\right) \kappa_{1}^{n+3}
$$

It follows that, in the regular case, relation (38) holds.

Singular case: If $\mu_{n-1}(0)=0$ then we can complete $\phi^{n-1}$ to a zero-preserving local diffeomorphism of $\mathbb{R}^{n}$ by taking $\phi^{n}=\left(\phi^{n-1}, \phi_{n}\right)^{T}$, where

$$
\phi_{n}(y)=\frac{\lambda_{n-1} y_{n}+\mu_{n-1}}{\nu_{n-1} y_{n}+\eta_{n-1}}
$$

Observe that $\mu_{n-1}(0)=0$ implies $\phi_{n}(0)=0$. Additionally, since $\mu_{n-1}(0)=0$ and $\left(\nu_{n-1} \mu_{n-1}-\lambda_{n-1} \eta_{n-1}\right)(0)$ $\neq 0$, we have $\lambda_{n-1}(0) \neq 0$ and $\eta_{n-1}(0) \neq 0$. In this case, we define

$$
\nu_{n}=\mathrm{L}_{\zeta_{1}^{n}} \phi_{n}, \quad \eta_{n}=\mathrm{L}_{\zeta_{2}^{n}} \phi_{n}, \quad \text { and } \quad \mu_{n}=\nu_{n-1} y_{n}+\eta_{n-1}
$$

Observe that $\nu_{n}(0)=\mathrm{L}_{\zeta_{1}^{n}} \phi_{n}(0)=\left(\lambda_{n-1} \eta_{n-1}-\nu_{n-1} \mu_{n-1}\right)(0) \neq 0$ and that $\mu_{n}(0)=\eta_{n-1}(0) \neq 0$. Thus the right hand side of (38) defines a locally invertible transformation. Moreover, the Kumpera-Ruiz normal form $\left(\kappa_{1}^{n}, \kappa_{2}^{n}\right)$ is defined to be the singular prolongation of $\left(\kappa_{1}^{n-1}, \kappa_{2}^{n-1}\right)$. 
Let us check that, again, relation (38) holds. Together, relations (39) and (40) give:

$$
\begin{aligned}
\phi_{*}^{n}\left(\zeta_{2}^{n}\right) & =\left(y_{n} \circ \psi^{n}\right) \phi_{*}^{n-1}\left(\zeta_{1}^{n-1}\right)+\phi_{*}^{n-1}\left(\zeta_{2}^{n-1}\right)+\left(\left(\mathrm{L}_{\zeta_{2}^{n}} \phi_{n}\right) \circ \psi^{n}\right) \frac{\partial}{\partial x_{n}} \\
& =\left(\left(\nu_{n-1} y_{n}+\eta_{n-1}\right) \circ \psi^{n}\right) \kappa_{1}^{n-1}+\left(\left(\lambda_{n-1} y_{n}+\mu_{n-1}\right) \circ \psi^{n}\right) \kappa_{2}^{n-1}+\left(\eta_{n} \circ \psi^{n}\right) \kappa_{1}^{n} \\
& =\left(\left(\nu_{n-1} y_{n}+\eta_{n-1}\right) \circ \psi^{n}\right)\left(\kappa_{1}^{n-1}+\left(\frac{\lambda_{n-1} y_{n}+\mu_{n-1}}{\nu_{n-1} y_{n}+\eta_{n-1}} \circ \psi^{n}\right) \kappa_{2}^{n-1}\right)+\left(\eta_{n} \circ \psi^{n}\right) \kappa_{1}^{n} \\
& =\left(\mu_{n} \circ \psi^{n}\right)\left(\kappa_{1}^{n-1}+x_{n} \kappa_{2}^{n-1}\right)+\left(\eta_{n} \circ \psi^{n}\right) \kappa_{1}^{n} \\
& =\left(\eta_{n} \circ \psi^{n}\right) \kappa_{1}^{n}+\left(\mu_{n} \circ \psi^{n}\right) \kappa_{2}^{n} .
\end{aligned}
$$

Like in the previous case, we have

$$
\phi_{*}^{n}\left(\zeta_{1}^{n}\right)=\left(\left(\mathrm{L}_{\zeta_{1}^{n}} \phi_{n}\right) \circ \psi^{n}\right) \frac{\partial}{\partial x_{n}}=\left(\nu_{n} \circ \psi^{n}\right) \kappa_{1}^{n+3} .
$$

It follows that relation (38) holds in both cases.

\section{Appendix B. Proof of Theorem 6.2 (abnormal Curves)}

We proceed now to the proof of Theorem 6.2, which states that an integral curve of $\mathcal{D}^{(i)}$ is locally abnormal if and only if it is an integral curve of $\mathcal{A}^{(i)}$. The proof will be based on the three following lemmas.

Lemma B.1. Consider a Goursat structure $\mathcal{D}$ defined on a manifold of dimension $n$, and fix an integer $i$ such that $0 \leq i \leq n-4$. An integral curve of $\mathcal{D}^{(i)}$ that has an empty intersection with the singular locus $K_{i}$ is locally abnormal if and only if it is an integral curve of $\mathcal{C}_{i}$, and thus of $\mathcal{A}^{(i)}$.

Proof of Lemma B.1. Let $\gamma: I \rightarrow M$ be an integral curve of $\mathcal{D}^{(i)}$ that does not intersect the singular locus $K_{i}$. Since we are outside $K_{i}$ it is easy to show, using a direct generalization of Goursat's normal form (see [32] and [43]), given by Theorem C.4, that for any fixed $t_{0}$ in $I$ we can find a local coordinate chart $x: U \rightarrow \mathbb{R}^{n}$ centered at $\gamma\left(t_{0}\right)$ and such that:

$$
\mathcal{D}^{(i)}=\left(\frac{\partial}{\partial x_{1}}, \ldots, \frac{\partial}{\partial x_{i+1}}, x_{i+1} \frac{\partial}{\partial x_{i+2}}+\cdots+x_{n-2} \frac{\partial}{\partial x_{n-1}}+\frac{\partial}{\partial x_{n}}\right) .
$$

Recall that $I_{\epsilon}(t)=I \cap\left[t_{0}-\epsilon, t_{0}+\epsilon\right]$. Chose a small enough $\varepsilon>0$ such that the restriction of $\gamma$ to $I_{\varepsilon}\left(t_{0}\right)$ is completely contained in the open set $U$. Then, the curve $x \circ \gamma: I_{\varepsilon}\left(t_{0}\right) \rightarrow \mathbb{R}^{n}$, which will be denoted shortly by $x(\cdot)$, is almost everywhere a solution of the following control system

$$
\begin{aligned}
\dot{x}_{1} & =u_{1} \\
& \vdots \\
\dot{x}_{i} & =u_{i} \\
\dot{x}_{i+1} & =u_{i+1} \\
\dot{x}_{i+2} & =x_{i+1} u_{i+2} \\
& \vdots \\
\dot{x}_{n-1} & =x_{n-2} u_{i+2} \\
\dot{x}_{n} & =u_{i+2} .
\end{aligned}
$$


Since the coordinate chart is centered at $\gamma\left(t_{0}\right)$, we have $x\left(t_{0}\right)=0$. The Hamiltonian of this system is given by

$$
H(x, p, u)=\sum_{k=1}^{i+1} p_{k} u_{k}+\sum_{k=i+2}^{n-1} p_{k} x_{k-1} u_{i+2}+p_{n} u_{i+2} .
$$

Therefore, the curve $x(\cdot)$ is abnormal if and only if there exists a non-trivial lift $(x(\cdot), p(\cdot))$ that satisfies, almost everywhere, the following differential equation

$$
\begin{aligned}
\dot{p}_{1} & =0 \\
& \vdots \\
\dot{p}_{i} & =0 \\
\dot{p}_{i+1} & =-p_{i+2} u_{i+2} \\
& \vdots \\
\dot{p}_{n-2} & =-p_{n-1} u_{i+2} \\
\dot{p}_{n-1} & =0 \\
\dot{p}_{n} & =0
\end{aligned}
$$

and, moreover, is such that $p_{k}=0$, for $1 \leq k \leq i+1$, and $p_{n}=-\sum_{k=i+2}^{n-1} p_{k} x_{k-1}$. The latter condition is a consequence of $\frac{\partial H}{\partial u}=0$.

Necessity. Assume that $x(\cdot)$ is not an integral curve of $\mathcal{C}_{i}$. We will prove that $x(\cdot)$ is not abnormal. In the coordinates of (41) we have $\mathcal{C}_{i}=\left(\frac{\partial}{\partial x_{1}}, \ldots, \frac{\partial}{\partial x_{i+1}}\right)$. Since $x(\cdot)$ is not an integral curve of $\mathcal{C}_{i}$, there exists a measurable subset $I_{0} \subset I_{\varepsilon}\left(t_{0}\right)$ such that the Lebesgue measure of $I_{0}$ is not zero and $u_{i+2}(t) \neq 0$ for each $t$ in $I_{0}$. If $x(\cdot)$ is abnormal then $p(\cdot)$ is such that $p_{i+1}(t)=0$ for each $t$ in $I_{\varepsilon}\left(t_{0}\right)$. Therefore, we have $\dot{p}_{i+1}=0$ almost everywhere on $I_{0}$. Indeed, note that if an absolutely continuous function $f$ on $I_{0}$ is such that $f(t)=0$ for almost all $t$ in $I_{0}$ then $f^{\prime}(t)=0$ for almost all $t$ in $I_{0}$. But $\dot{p}_{i+1}=-p_{i+2} u_{i+2}$ and $u_{i+2} \neq 0$ imply $p_{i+2}=0$ almost everywhere on $I_{0}$, which gives $\dot{p}_{i+2}=0$ almost everywhere on $I_{0}$. We can repeat the previous argument to obtain $p_{k}=0$, for $1 \leq k \leq n-1$, almost everywhere on $I_{0}$. Since $p_{n}=-\sum_{k=i+2}^{n-1} p_{k} x_{k-1}$, we have also $p_{n}=0$ almost everywhere on $I_{0}$. This gives $p_{k}=0$, almost everywhere on $I_{0}$, for $1 \leq k \leq n$, which is impossible since $p$ must be non-trivial.

Sufficiency. Now, assume that $x(\cdot)$ is an integral curve of $\mathcal{C}_{i}$. In order to prove that $x(\cdot)$ is abnormal, we will consider the lift defined by $p_{k}=0$ for $1 \leq k \leq n$, with the exception of $p_{n-1}$, for which any non-zero real constant can be taken. Since $x(\cdot)$ is an integral curve of $\mathcal{C}_{i}$ we must have $u_{i+2}(t)=0$ almost everywhere on $I_{\varepsilon}\left(t_{0}\right)$, which implies that $p(\cdot)$ satisfies (42). Moreover, since $x\left(t_{0}\right)=0$, we have $x_{k}(t)=x_{k}\left(t_{0}\right)=0$, for each $t$ in $I_{\varepsilon}\left(t_{0}\right)$ and for $i+2 \leq k \leq n$. Thus $p_{n}$, which was taken to be zero, satisfies $p_{n}=-\sum_{k=i+2}^{n-1} p_{k} x_{k-1}$ (recall that $\left.p_{i+2}=0\right)$. In other words $p(\cdot)$ satisfies $\frac{\partial H}{\partial u}=0$. Finally, since $p_{n-1} \neq 0$, our lift is non-trivial, which implies that $x(\cdot)$ is abnormal.

Lemma B.2. Consider a Goursat structure $\mathcal{D}$ defined on a manifold of dimension $n$ and fix an integer $i$ such that $0 \leq i \leq n-5$. Let $x(\cdot)$ be the restriction of an integral curve of $\mathcal{D}^{(i)}$ to the interval $I_{\varepsilon}\left(t_{0}\right)$, where $\varepsilon>0$. If a fixed measurable subset $I_{0} \subset \mathbb{R}$ is such that for each $t$ in $I_{0} \cap I_{\varepsilon}\left(t_{0}\right)$ we have $\dot{x}(t) \notin \mathcal{A}^{(i)}(x(t))$ then, for a small enough $\varepsilon>0$, we have $x(t) \notin K_{i}$ for almost all $t$ in $I_{0} \cap I_{\varepsilon}\left(t_{0}\right)$.

Proof of Lemma B.2. Let $x: I_{\varepsilon}\left(t_{0}\right) \rightarrow \mathbb{R}^{n}$ be the restriction to the interval $I_{\varepsilon}\left(t_{0}\right)$, where $\varepsilon>0$, of an integral curve of $\mathcal{D}^{(i)}$. Firstly, if $x\left(t_{0}\right) \notin K_{i}$ then there exists a small enough $\varepsilon$ such that $x(\cdot)$ does not intersect $K_{i}$ and thus, in this case, the lemma is trivially true. Secondly, if the Lebesgue measure of $I_{0}$ is 0 then the lemma 
is also trivially true. Finally, if the closure of $I_{0}$ does not contain $t_{0}$ then for a small enough $\varepsilon$ the Lebesgue measure of $I_{0} \cap I_{\varepsilon}\left(t_{0}\right)$ will be 0 and thus the lemma will be, once more, trivially true. Hence, from now on, we will only consider curves such that $x\left(t_{0}\right)$ belongs to $K_{i}$, the Lebesgue measure of $I_{0}$ is not 0 , and the closure of $I_{0}$ contains $t_{0}$. Moreover, once a small enough $\varepsilon>0$ has been fixed, we will denote also by $I_{0}$ the intersection $I_{0} \cap I_{\varepsilon}\left(t_{0}\right)$. That is, we will assume that $I_{0} \subset I_{\varepsilon}\left(t_{0}\right)$.

For any such integral curve $x(\cdot)$ of $\mathcal{D}^{(i)}$ it is easy to prove, using a direct generalization of Kumpera-Ruiz's normal form, given by Theorem C.5 (with a double indexation of coordinates, like in Cor. 2.4), that there exist coordinates on $\mathbb{R}^{n}$ in which $x(\cdot)$ is a solution of the following control system:

$$
\begin{aligned}
\dot{x}_{1}^{0} & =u_{1} \\
\dot{x}_{2}^{0} & =u_{2} \\
\vdots & \\
\dot{x}_{i}^{0} & =u_{i} \\
\dot{x}_{i+1}^{0} & =u_{i+1} \\
\dot{x}_{q}^{p} & =\left(\prod_{0 \leq r \leq p-1} x_{k_{r}}^{r}\right)\left(x_{q-1}^{p}+c_{q-1}^{p}\right) u_{i+2} \quad \text { for } \quad i+2 \leq q \leq k_{0} \text { if } p=0 \text { and } \quad 2 \leq q \leq k_{p} \text { if } 1 \leq p \leq m \\
\dot{x}_{1}^{p+1} & =\left(\prod_{0 \leq r \leq p-1} x_{k_{r}}^{r}\right) u_{i+2} \quad \text { for } \quad 0 \leq p \leq m,
\end{aligned}
$$

where $x=\left(x_{1}^{0}, x_{2}^{0}, \ldots, x_{k_{0}}^{0}, x_{1}^{1}, \ldots, x_{k_{m}}^{m}, x_{1}^{m+1}\right)$ and $x\left(t_{0}\right)=0$ (recall that we assume that $x\left(t_{0}\right)$ belongs to the singular locus, which means that $m \geq 1$ ). Moreover, the integers $k_{i}$ that appear in (43) satisfy $i+1 \leq k_{0} \leq n-4$ and $k_{1} \geq 1, \ldots, k_{m-1} \geq 1, k_{m} \geq 3, k_{m+1}=1$ and $\sum_{r=0}^{m+1} k_{r}=n$. Observe that the number $m \geq 1$ is the number of singularities of $\mathcal{D}^{(i)}$, which can be smaller than the number of singularities of $\mathcal{D}$. In these coordinates, the singular locus is given by

$$
K_{i}=\left\{\prod_{r=0}^{m-1} x_{k_{r}}^{r}=0\right\}
$$

If $c_{q}^{0}=0$, for all $i+1 \leq q \leq k_{0}-1$, then the only integer $j$ such that $S_{j}^{(i)}$ contains zero is $j=k_{0}-(i+1)$. Thus $L_{i}$ is given (see Prop. 4.4) by

$$
L_{i}=S_{k_{0}-1-i}^{\left(k_{0}-1\right)}=\left\{x_{i+1}^{0}=x_{i+2}^{0}=\cdots=x_{k_{0}}^{0}=0\right\} .
$$

Note that if for some $i+1 \leq q \leq k_{0}-1$ we have $c_{q}^{0} \neq 0$ then the submanifold $L_{i}$ does not contain zero (it is locally empty at zero).

For each point $p$ of $\mathbb{R}^{n}$, we have

$$
\mathcal{C}_{i}(p)=\left(\frac{\partial}{\partial x_{1}^{0}}, \ldots, \frac{\partial}{\partial x_{i+1}^{0}}\right)(p)
$$

If $c_{q}^{0}=0$, for $i+1 \leq q \leq k_{0}-1$, then we have

$$
\mathcal{A}^{(i)}(p)=\left(\frac{\partial}{\partial x_{1}^{0}}, \ldots, \frac{\partial}{\partial x_{i+1}^{0}}\right)(p) \cup\left(\frac{\partial}{\partial x_{1}^{0}}, \ldots, \frac{\partial}{\partial x_{i}^{0}}, \frac{\partial}{\partial x_{1}^{1}}\right)(p),
$$


for each point $p$ in $L_{i}$ and $\mathcal{A}^{(i)}(p)=\mathcal{C}_{i}(p)$, outside $L_{i}$. If for some $i+1 \leq q \leq k_{0}-1$ we have $c_{q}^{0} \neq 0$ then, in a small enough neighborhood $U$ of zero, we have $\mathcal{A}^{(i)}(p)=\mathcal{C}_{i}(p)$ for each point $p$ in $U$.

Recall that the integral curve $x: I_{\varepsilon}\left(t_{0}\right) \rightarrow \mathbb{R}^{n}$ of $\mathcal{D}^{(i)}$ is such that $x\left(t_{0}\right)=0$. Assume that, at a given $t$ of $I_{\varepsilon}\left(t_{0}\right)$, the velocity $\dot{x}(t)$ exists and satisfies (43). Then, if $\varepsilon$ is small enough, we have by the above analysis that the velocity $\dot{x}(t)$ belongs to $\mathcal{A}^{(i)}(x(t))$ if and only if we have $u_{i+2}(t)=0$ or the three following conditions hold: (i) $u_{i+1}(t)=0$ and (ii) $x_{q}^{0}(t)=0$, for $i+1 \leq q \leq k_{0}$, and (iii) $c_{q}^{0}=0$, for $i+1 \leq q \leq k_{0}-1$.

Now, suppose that for each $t$ in $I_{0} \subset I_{\varepsilon}\left(t_{0}\right)$ the velocity $\dot{x}(t)$ exists and is such that $\dot{x}(t) \notin \mathcal{A}^{(i)}(x(t))$. Recall that we can assume that the Lebesgue measure of $I_{0}$ is not 0 and the closure of $I_{0}$ contains $t_{0}$. For each $t$ in $I_{0}$ we have $u_{i+2}(t) \neq 0$. Additionally: (a) If $c_{q}^{0}=0$ for $i+1 \leq q \leq k_{0}-1$ then we can represent the subset $I_{0}$ as $I_{0}=I_{1} \cup I_{2}$ (with, in general, a non empty intersection of $I_{1}$ and $I_{2}$ ), where $I_{1}$ is the set of points where (i) is not satisfied and $I_{2}$ is the set of points where (ii) is not satisfied. (b) If there exists an integer $i+1 \leq q \leq k_{0}-1$ such that $c_{q}^{0} \neq 0$ then $I_{0}=\left\{t \in I_{\varepsilon}\left(t_{0}\right): u_{i+2}(t) \neq 0\right\}$, provided that $\varepsilon$ is small enough. We are going to show that, in both cases, we have $x(t) \notin K_{i}$, for almost all $t$ in $I_{0}$.

Case (a): Subset $I_{1}$. For each $t$ in $I_{1}$ we have both $u_{i+2}(t) \neq 0$ and $u_{i+1}(t) \neq 0$. Therefore, we have $\dot{x}_{i+1}^{0} \neq 0$ almost everywhere on $I_{1}$, which implies that $x_{i+1}^{0} \neq c_{i+1}^{0}$ almost everywhere on $I_{1}$. Indeed, note that if an absolutely continuous function $f$ on $I_{1}$ is such that $f^{\prime}(t) \neq 0$ for almost all $t$ in $I_{1}$ then, for any constant $c$, the measure of the set $\left\{t \in I_{1}: f(t)=c\right\}$ is zero.

Now, using an induction argument we will show, successively, that $x_{i+1}^{0} \neq c_{i+1}^{0}, x_{i+2}^{0} \neq c_{i+2}^{0}, \ldots, x_{k_{0}-1}^{0} \neq$ $c_{k_{0}-1}^{0}, x_{k_{0}}^{0} \neq 0, x_{1}^{1} \neq c_{1}^{1}, \ldots, x_{k_{1}-1}^{1} \neq c_{k_{1}-1}^{1}, x_{k_{1}}^{1} \neq 0, \ldots, x_{k_{m-1}}^{m-1} \neq 0$, almost everywhere on $I_{1}$. Suppose that this assumption is true up to $x_{q-1}^{p}$. We have two cases: either $q \leq k_{p}$ or $q=k_{p}+1$. If $q \leq k_{p}$ then $\dot{x}_{q}^{p}=\prod_{0 \leq r \leq p-1}\left(x_{k_{r}}^{r}\right)\left(x_{q-1}^{p}+c_{q-1}^{p}\right) u_{i+2}$. Since $x_{k_{r}}^{r} \neq 0$, for $0 \leq r \leq p-1$, and $x_{q-1}^{p} \neq c_{q-1}^{p}$ and $u_{i+2} \neq 0$, almost everywhere on $I_{1}$, we have $\dot{x}_{q}^{p}(t) \neq 0$ for almost all $t$ in $I_{1}$. This implies, almost everywhere on $I_{1}$, that $x_{q}^{p} \neq c_{q}^{p}$ if $q \leq k_{p}-1$ or that $x_{q}^{p} \neq 0$ if $q=k_{p}$. Otherwise $q=k_{p}+1$ and in this case $\dot{x}_{1}^{p+1}=\prod_{0 \leq r \leq p-1}\left(x_{k_{r}}^{r}\right) u_{i+2}$. Since $x_{k_{r}}^{r} \neq 0$, for $0 \leq r \leq p-1$, and $u_{i+2} \neq 0$, almost everywhere on $I_{1}$, we have $\dot{x}_{1}^{p+1}(t) \neq 0$ for almost all $t$ in $I_{1}$, which implies $x_{1}^{p+1} \neq c_{1}^{p+1}$ almost everywhere on $I_{1}$. This ends the induction argument. In particular, we have proved that $x_{k_{r}}^{r}(t) \neq 0$ for almost all $t$ in $I_{1}$, for each $0 \leq r \leq m-1$. Now, recall that the singular locus is given by the relation $\prod_{r=0}^{m-1} x_{k_{r}}^{r}=0$. It thus follows that we have $x(t) \notin K_{i}$ for almost all $t$ in $I_{1}$.

Case (a): Subset $I_{2}$. We can represent the subset $I_{2}$ as $I_{2}=I_{2}^{i+1} \cup \cdots \cup I_{2}^{k_{0}}$, where $I_{2}^{q}=\left\{t \in I_{0}: x_{q}^{0}(t) \neq 0\right\}$. Observe that, in general, the intersection of these subsets will be non empty. Now on each subset $I_{2}^{q}$, of positive Lebesgue measure, we can follow the same proof as for the subset $I_{1}$, starting the induction argument with $x_{q}^{0}$. For each one of these subsets the conclusion is the same: we have $x(t) \notin K_{i}$ for almost all $t$ in $I_{2}^{q}$.

Case (b): We have $u_{i+2}(t) \neq 0$ for each $t$ in $I_{0}$ and, moreover, there exists $q$ such that $c_{q}^{0} \neq 0$, where $i+1 \leq q \leq$ $k_{0}-1$. Since $c_{q}^{0} \neq 0$, we can take a smaller $\varepsilon>0$, if necessary, in order to have $\dot{x}_{q+1}^{0}(t)=\left(x_{q}^{0}(t)+c_{q}^{0}\right) u_{i+2} \neq 0$ for each $t$ in $I_{0}$. This implies that $x_{q+1}^{0} \neq c_{q+1}^{0}$ almost everywhere on $I_{0}$. The rest of the proof follows like for the subset $I_{1}$ and the conclusion is the same: we have $x(t) \notin K_{i}$ for almost all $t$ in $I_{0}$. The only difference being that the induction argument starts with $x_{q+1}^{0}$ instead of $x_{i+1}^{0}$.

Lemma B.3. Consider a Goursat structure $\mathcal{D}$ defined on a manifold of dimension $n$ and fix an integer $i$ such that $0 \leq i \leq n-5$. An integral curve of $\mathcal{A}^{(i)}$ that intersects the singular locus $K_{i}$ is locally, in a small enough neighborhood of any point of intersection, an abnormal curve of $\mathcal{D}^{(i)}$.

Proof of Lemma B.3. Let $x: I_{\varepsilon}\left(t_{0}\right) \rightarrow \mathbb{R}^{n}$ be the restriction to the interval $I_{\varepsilon}\left(t_{0}\right)$, where $\varepsilon>0$, of an integral curve of $\mathcal{D}^{(i)}$ that intersects the singular locus at $x\left(t_{0}\right)$. It is easy to prove (see Lem. D.2) that, for a small enough $\varepsilon>0$, there exist coordinates such that the integral curve $x(\cdot)$ is a solution of the following control 
system:

$$
\begin{aligned}
\dot{x}_{1} & =u_{1} \\
\vdots & \\
\dot{x}_{i} & =u_{i} \\
\dot{x}_{i+1} & =u_{i+1} \\
\dot{x}_{i+2} & =\left(x_{i+1}+c_{i+1}\right) u_{i+2} \\
\vdots & \\
\dot{x}_{k_{0}} & =\left(x_{k_{0}-1}+c_{k_{0}-1}\right) u_{i+2} \\
\dot{x}_{k_{0}+1} & =x_{k_{0}} u_{i+2} \\
\dot{x}_{k_{0}+2} & =u_{i+2} \\
\dot{x}_{k_{0}+3} & =x_{k_{0}} x_{k_{0}+2} u_{i+2} \\
\dot{x}_{k_{0}+4} & =x_{k_{0}} x_{k_{0}+3} u_{i+2} \\
\dot{x}_{j} & =x_{k_{0}} \phi_{j}\left(\bar{x}_{k_{0}+3}\right) u_{i+2} \quad \text { for } k_{0}+5 \leq j \leq n,
\end{aligned}
$$

where $x=\left(x_{1}, x_{2}, \ldots, x_{n}\right)$ and $\bar{x}_{k_{0}+3}=\left(x_{k_{0}+3}, x_{k_{0}+4}, \ldots, x_{n}\right)$. Since $x\left(t_{0}\right)$ belongs to $K_{i}$, we can assume that $x\left(t_{0}\right)=0$. Moreover, like in the proof of the previous lemma, we have $i+1 \leq k_{0} \leq n-4$.

The Hamiltonian of this system is given by

$$
\begin{aligned}
H(x, p, u)= & \sum_{j=1}^{i+1} p_{j} u_{j}+\sum_{j=i+2}^{k_{0}} p_{j}\left(x_{j-1}+c_{j-1}\right) u_{i+2}+p_{k_{0}+1} x_{k_{0}} u_{i+2}+p_{k_{0}+2} u_{i+2} \\
& +\left(p_{k_{0}+3} x_{k_{0}+2}+p_{k_{0}+4} x_{k_{0}+3}+\sum_{j=k_{0}+5}^{n} p_{j} \phi_{j}\left(\bar{x}_{k_{0}+3}\right)\right) x_{k_{0}} u_{i+2},
\end{aligned}
$$

which implies that any abnormal lift $(x(\cdot), p(\cdot))$ of $x(\cdot)$ must satisfy

$$
\begin{aligned}
\dot{p}_{j} & =0 \quad \text { for } 1 \leq j \leq i \\
\dot{p}_{j} & =-p_{j+1} u_{i+2} \quad \text { for } i+1 \leq j \leq k_{0}-1 \\
\dot{p}_{k_{0}} & =\left(-p_{k_{0}+1}-p_{k_{0}+3} x_{k_{0}+2}-p_{k_{0}+4} x_{k_{0}+3}-\sum_{j=k_{0}+5}^{n} p_{j} \phi_{j}\left(\bar{x}_{k_{0}+3}\right)\right) u_{i+2} \\
\dot{p}_{k_{0}+1} & =0 \\
\dot{p}_{j} & =-\psi_{j}\left(\bar{x}_{k_{0}+3}, p\right) x_{k_{0}} u_{i+2} \quad \text { for } k_{0}+2 \leq j \leq n,
\end{aligned}
$$

where the $\psi_{j}$ 's are some functions of $x_{k_{0}+3}, x_{k_{0}+4}, \ldots, x_{n}$ and $p_{k_{0}+3}, p_{k_{0}+4}, \ldots, p_{n}$, for $k_{0}+2 \leq j \leq n$. The exact form of these functions is irrelevant for our purpose. Any abnormal lift $(x(\cdot), p(\cdot))$ of $x(\cdot)$ must also satisfy the relation $\frac{\partial H}{\partial u}=0$, which implies $p_{j}=0$, for $1 \leq j \leq i+1$, and

$$
p_{k_{0}+2}=-\sum_{j=i+2}^{k_{0}} p_{j}\left(x_{j-1}+c_{j-1}\right)+-\left(p_{k_{0}+1}+p_{k_{0}+3} x_{k_{0}+2}+p_{k_{0}+4} x_{k_{0}+3}+\sum_{j=k_{0}+5}^{n} p_{j} \phi_{j}\left(\bar{x}_{k_{0}+3}\right)\right) x_{k_{0}} .
$$

Recall that $x(\cdot)$ is an integral curve of $\mathcal{D}^{(i)}$. Like in the proof of Lemma B.2, if for a given $t$ the velocity $\dot{x}(t)$ exists and satisfies (44) then we have $\dot{x}(t) \in \mathcal{A}^{(i)}(x(t))$ if and only if $u_{i+2}(t)=0$ or the three following conditions 
hold: (i) $u_{i+1}(t)=0$ and (ii) $x_{j}(t)=0$, for $i+1 \leq j \leq k_{0}$, and (iii) $c_{j}=0$, for $i+1 \leq j \leq k_{0}-1$. Now, assume that $x(\cdot)$ is an integral curve of $\mathcal{A}^{(i)}$, that is $\dot{x}(t) \in \mathcal{A}^{(i)}(x(t))$ for almost all $t$ in $I_{\varepsilon}\left(t_{0}\right)$. In order to prove that $x(\cdot)$ is abnormal we must construct a non-trivial abnormal lift $(x(\cdot), p(\cdot))$ of $x(\cdot)$. Take $p_{j}=0$, for $1 \leq j \leq n$, with the exception of $p_{k_{0}+4}$, for which we take any non-zero real constant. It is straightforward to check that our lift satisfies (45). Indeed, the coordinate $x_{k_{0}+3}$ is constant because we have $\dot{x}_{k_{0}+3}=x_{k_{0}} x_{k_{0}+2} u_{i+2} ;$ and $u_{i+2}(t)=0$ or $x_{k_{0}}(t)=0$ for almost all $t$. Moreover, since $x(0)=0$, we have $x_{k_{0}+3}(t)=0$, for each $t$ in $I_{\varepsilon}\left(t_{0}\right)$. It is also trivial to check that our lift satisfies $\frac{\partial H}{\partial u}=0$. Since $p_{k_{0}+4} \neq 0$ our lift in non-trivial. It follows that the integral curve $x(\cdot)$ is abnormal.

Proof of Theorem 6.2. Let $x: I_{\varepsilon}\left(t_{0}\right) \rightarrow \mathbb{R}^{n}$ be the restriction to the interval $I_{\varepsilon}\left(t_{0}\right)$ of an integral curve of $\mathcal{D}^{(i)}$. For $\varepsilon>0$ small enough, we can apply both Lemma B.1 and Lemma B.3, which imply that if the curve $x(\cdot)$ is such that $\dot{x}(t)$ belongs to $\mathcal{A}^{(i)}(x(t))$ for almost all $t$ in $I_{\varepsilon}\left(t_{0}\right)$ then $x(\cdot)$ is abnormal. In other words, the integral curves of $\mathcal{A}^{(i)}$ are locally abnormal.

Now assume that, for a fixed interval $I_{\varepsilon}\left(t_{0}\right)$, the curve $x(\cdot)$ is abnormal. Define the subset $I_{0} \subset I_{\varepsilon}\left(t_{0}\right)$ by

$$
I_{0}=\left\{t \in I_{\varepsilon}\left(t_{0}\right): \dot{x}(t) \text { exists and } \dot{x}(t) \notin \mathcal{A}^{(i)}(x(t)\} .\right.
$$

We will show that if $\varepsilon$ is small enough then the Lebesgue measure of $I_{0}$ is zero. We can decompose $I_{0}$ into $I_{0}=I_{1} \cup I_{2}$, where

$$
I_{1}=\left\{t \in I_{0}: x(t) \in K_{i}\right\} \quad \text { and } \quad I_{2}=\left\{t \in I_{0}: x(t) \notin K_{i}\right\}
$$

On the one hand, the measure of $I_{1}$ is equal to zero. Indeed, since for each $t$ in $I_{0}$ we have $\dot{x}(t) \notin \mathcal{A}^{(i)}(x(t))$, for $\varepsilon$ small enough Lemma B.2 implies that we have $x(t) \notin K_{i}$ for almost all $t$ in $I_{0}$. But, on the other hand, the measure of $I_{2}$ is also equal to zero. To see this, let us write $I_{2}$ as $I_{2}=I_{0} \cap I_{3}$, where

$$
I_{3}=\left\{t \in I_{\varepsilon}\left(t_{0}\right): x(t) \notin K_{i}\right\}
$$

Since $K_{i}$ is closed (see the discussion following the statement of Th. 6.2) and $x(\cdot)$ is continuous, it is clear that we can decompose $I_{3}$ into a union of disjoint open intervals $I_{3}=\bigcup J_{\alpha}$ such that, on each of them, the curve $x(\cdot)$ has an empty intersection with the singular locus $K_{i}$. Moreover, since the set $I_{3}$ is an open subset of $\mathbb{R}$ the union can be taken to be countable. Now Lemma B.1 implies that, for each $\alpha$, we have $\dot{x}(t) \in \mathcal{A}^{(i)}(x(t))$ for almost all $t$ in $J_{\alpha}$ because $x(\cdot)$ is abnormal and we are outside the singular locus. Hence, since the measure of $I_{2}$ is the sum of the measures of the sets $I_{0} \cap J_{\alpha}$ (the union is countable) and the measure of each of these sets is zero, the measure of $I_{2}$ equals zero.

\section{Appendix C. Weber's PROBLEM}

Our proof of Kumpera-Ruiz's theorem was based on the following fact: if a rank two distribution $\mathcal{D}$ on a manifold $M$ of dimension $n \geq 4$ satisfies $\operatorname{dim} \mathcal{D}^{(1)}(p)=3$ and $\operatorname{dim} \mathcal{D}^{(2)}(p)=4$, for each point $p$ in $M$, then there exists a canonical line field $\mathcal{L} \subset \mathcal{D}$ that satisfies $\left[\mathcal{L}, \mathcal{D}^{(1)}\right] \subset \mathcal{D}^{(1)}$. This observation has a natural generalization: if a rank $k \geq 2$ distribution $\mathcal{D}$ on a manifold $M$ of dimension $n \geq k+2$ satisfies $\operatorname{dim} \mathcal{D}^{(1)}(p)=k+1$ and $\operatorname{dim} \mathcal{D}^{(2)}(p)=k+2$, for each point $p$ in $M$, then there exists (i) a canonical involutive distribution $\mathcal{L}_{1} \subset \mathcal{D}^{(0)}$ that has rank $k-1$ and is uniquely characterized by $\left[\mathcal{L}_{1}, \mathcal{D}^{(1)}\right] \subset \mathcal{D}^{(1)}$; and (ii) a canonical involutive distribution $\mathcal{L}_{0} \subset \mathcal{D}^{(0)}$ that has rank $k-2$ and is uniquely characterized by $\left[\mathcal{L}_{0}, \mathcal{D}^{(0)}\right] \subset \mathcal{D}^{(0)}$ (see [76], as well as [32] and [43] for an approach based on Pfaffian systems; see also [31] and Prop. 4.1).

Though the above observation appears more or less clearly in the work of Cartan (see e.g. [11]; see also [22]), its origin can be found in the pioneering work of Engel [16], for $n=k+2$, and von Weber [72], for $n \geq k+2$ (see also [9]). This observation is closely related to the following result, which is clearly stated in Weber's article ([72], Th. V) (using the dual language of Pfaffian systems). 
Theorem C.1 (von Weber). Let $\mathcal{D}$ be a rank $k \geq 2$ distribution on a manifold $M$ of dimension $n=m+k-2$ $\geq 4$. Assume that $\operatorname{dim} \mathcal{D}^{(1)}(p)=k+1$ and $\operatorname{dim} \mathcal{D}^{(2)}(p)=k+2$, for each point $p$ in $M$. Then, in a small enough neighborhood of any point $p$ in $M$, the distribution $\mathcal{D}$ is equivalent to a distribution spanned by a family of vector fields that has the following form:

$$
\left(\frac{\partial}{\partial x_{m+k-2}}, \ldots, \frac{\partial}{\partial x_{m+1}}, \frac{\partial}{\partial x_{m}}, x_{m} \frac{\partial}{\partial x_{m-1}}+\sum_{i=2}^{m-2} \varphi_{i}\left(\bar{x}_{m-1}\right) \frac{\partial}{\partial x_{i}}+\frac{\partial}{\partial x_{1}}\right),
$$

where the functions $\varphi_{i}$, for $2 \leq i \leq m-2$, depend on the variables $x_{1}, \ldots, x_{m-1}$ only.

The following result is a direct consequence of Theorem C.1.

Proposition C.2. Any Goursat structure on a manifold $M$ of dimension $n \geq 4$ is equivalent, in a small enough neighborhood of any point $p$ in $M$, to a distribution spanned by a pair of vector fields that has the following form:

$$
\left(\frac{\partial}{\partial x_{n}}, x_{n} \frac{\partial}{\partial x_{n-1}}+x_{n-1} \frac{\partial}{\partial x_{n-2}}+\sum_{i=2}^{n-3} \varphi_{i}\left(\bar{x}_{n-1}\right) \frac{\partial}{\partial x_{i}}+\frac{\partial}{\partial x_{1}}\right)
$$

where the coordinates $x_{1}, \ldots, x_{n}$ are centered at $p$ and the functions $\varphi_{i}$, for $2 \leq i \leq n-3$, depend on the variables $x_{1}, \ldots, x_{n-1}$ only.

In the particular case of four-manifolds the last result gives:

Corollary C.3 (Engel's theorem). Any Goursat structure on a four-manifold $M$ is equivalent, in a small enough neighborhood of any point $p$ in $M$, to the distribution spanned by the following pair of vector fields (Engel's normal form):

$$
\left(\frac{\partial}{\partial x_{4}}, x_{4} \frac{\partial}{\partial x_{3}}+x_{3} \frac{\partial}{\partial x_{2}}+\frac{\partial}{\partial x_{1}}\right)
$$

where the $x$-coordinates are centered at $p$.

The following theorem can be considered as a rigorous version of Weber's result ([72], Th. VI), it is a direct consequence of the work of Kumpera and Ruiz [32], Martin and Rouchon [43], Murray [53], and Zhitomirskiu [76] (see also [46]).

Theorem C.4 (Weber's problem). A rank $k \geq 2$ distribution $\mathcal{D}$ on a manifold $M$ of dimension $n=m+k-2$ $\geq 4$ is equivalent, in a small enough neighborhood of a given point $p$ in $M$, to the distribution spanned by the following family of vector fields (Weber's normal form)

$$
\left(\frac{\partial}{\partial x_{m+k-2}}, \ldots, \frac{\partial}{\partial x_{m+1}}, \frac{\partial}{\partial x_{m}}, x_{m} \frac{\partial}{\partial x_{m-1}}+\cdots+x_{3} \frac{\partial}{\partial x_{2}}+\frac{\partial}{\partial x_{1}}\right)
$$

if and only if $\operatorname{dim} \mathcal{D}_{i}(p)=\operatorname{dim} \mathcal{D}^{(i)}(p)=k+i$, for $0 \leq i \leq m-2$, in a small enough neighborhood of $p$.

If we have $\operatorname{dim} \mathcal{D}^{(i)}(p)=k+i$, for $0 \leq i \leq n-2$, but we do not impose any condition on $\operatorname{dim} \mathcal{D}_{i}(p)$ then we still have the following result (see also [46]), which is a direct consequence of Theorem C.1 and Theorem 2.3, applied to the last two vectors fields of (46).

Theorem C.5 (Kumpera-Ruiz). Let $\mathcal{D}$ be a rank $k \geq 2$ distribution on a manifold $M$ of dimension $n=$ $m+k-2 \geq 4$, such that for any point $p$ in $M$ we have $\operatorname{dim} \mathcal{D}^{(i)}(p)=k+i$, for $0 \leq i \leq m-2$. Then, the 
distribution $\mathcal{D}$ is equivalent, in a small enough neighborhood of any point $p$ in $M$, to the distribution spanned by the following family of vector fields:

$$
\left(\frac{\partial}{\partial x_{m+k-2}}, \ldots, \frac{\partial}{\partial x_{m+1}}, \kappa_{1}^{m}, \kappa_{2}^{m}\right)
$$

where the pair of vector fields $\left(\kappa_{1}^{m}, \kappa_{2}^{m}\right)$ denotes a Kumpera-Ruiz normal form on $\mathbb{R}^{m}$.

\section{Appendix D. Additional normal Forms}

Let $\xi^{m}=\left(\xi_{1}^{m}, \xi_{2}^{m}\right)$ be a pair of vector fields defined on $\mathbb{R}^{m}$ that has the following form:

$$
\left(\frac{\partial}{\partial x_{m}}, x_{m} \frac{\partial}{\partial x_{m-1}}+x_{m-1} \frac{\partial}{\partial x_{m-2}}+\sum_{i=2}^{m-3} \varphi_{i}\left(\bar{x}_{m-1}\right) \frac{\partial}{\partial x_{i}}+\frac{\partial}{\partial x_{1}}\right) .
$$

A pair of vector fields $\xi^{m+l}=\left(\xi_{1}^{m+l}, \xi_{2}^{m+l}\right)$ defined on $\mathbb{R}^{m+l}$, for $l \geq 0$, is called a prolongation of order $l$ of $\xi^{m}$ if we have $\xi^{m+l}=\sigma_{l} \circ \cdots \circ \sigma_{1}\left(\xi^{m}\right)$, where each $\sigma_{i}$, for $1 \leq i \leq l$, equals either $S$ or $R_{c_{i}}$, for some real constants $c_{i}$ (recall that the singular and regular prolongations $S$ and $R_{c_{i}}$ have been defined in Sect. 2).

The following lemma is a natural generalization of Proposition C.2.

Lemma D.1. Let $\mathcal{D}$ be a rank $k \geq 2$ distribution on a manifold $M$ of dimension $n=m+l+k-2 \geq 4$, where $l$ and $m$ are two non-negative integers. Assume that for each point $p$ in $M$ we have $\operatorname{dim} \mathcal{D}^{(i)}(p)=k+i$, for $0 \leq i \leq l+2$. Then, in a small enough neighborhood of any point $p$ in $M$, the distribution $\mathcal{D}$ is equivalent to a distribution spanned by a family of vector fields that has the following form:

$$
\left(\frac{\partial}{\partial x_{m+l+k-2}}, \ldots, \frac{\partial}{\partial x_{m+l+1}}, \xi_{1}^{m+l}, \xi_{2}^{m+l}\right)
$$

where the pair of vector fields $\left(\xi_{1}^{m+l}, \xi_{2}^{m+l}\right)$ is a prolongation of order l of a pair of vector fields $\left(\xi_{1}^{m}, \xi_{2}^{m}\right)$ of the form (49).

The proof of Lemma D.1 is left to the reader. For generic points, the lemma is stated and proved in the work of Cartan [11] and Goursat [22]. For singular points, the lemma is a direct consequence of the results obtained by Kumpera and Ruiz [32] and its proof is almost the same as that of Theorem 2.3 but there are essentially two differences. The first difference is that instead of using Proposition A.1, as it is done in the proof of Theorem 2.3, one uses Theorem C.1; the second difference is that instead of starting the induction argument, for $l=0$, with the Pfaff-Darboux normal form, as it is done in the proof of Theorem 2.3, one starts it with Weber's preliminary normal form (49).

Let $\mathcal{D}$ be a rank $k \geq 2$ distribution on a manifold $M$ of dimension $n=m+k-2 \geq 4$, such that for any point $p$ in $M$ we have $\operatorname{dim} \mathcal{D}^{(i)}(p)=k+i$, for $0 \leq i \leq m-2$. It is easy to check that each distribution $\mathcal{D}^{(i)}$, for $0 \leq i \leq m-4$, contains a unique involutive subdistribution $\mathcal{C}_{i} \subset \mathcal{D}^{(i)}$ that has constant corank one in $\mathcal{D}^{(i)}$ and is characteristic for $\mathcal{D}^{(i+1)}$. We can generalize the canonical submanifolds $S_{0}^{(i)}$ of Section 4 by the following definition:

$$
S_{0}^{(i)}=\left\{p \in M: \mathcal{D}^{(i)}(p)=\mathcal{C}_{i+1}(p)\right\}
$$

where $0 \leq i \leq m-5$. We say that a point $p$ of $M$ is singular if there exists an integer $0 \leq i \leq m-5$ such that $p \in S_{0}^{(i)}$. For a singular point $p$, we denote by $k_{0}$ the smallest integer $1 \leq k_{0} \leq m-4$ such that $p \in S_{0}^{\left(k_{0}-1\right)}$.

Lemma D.2. Let $\mathcal{D}$ be a rank $k \geq 2$ distribution on a manifold $M$ of dimension $n=m+k_{0}+k-2 \geq 4$, such that for any point $p$ in $M$ we have $\operatorname{dim} \mathcal{D}^{(i)}(p)=k+i$, for $0 \leq i \leq k_{0}+2$. Assume, moreover, that $k_{0}$ is the 
smallest integer such that $\mathcal{D}^{\left(k_{0}-1\right)}(p)=\mathcal{C}_{k_{0}}(p)$. Then, in a small enough neighborhood of $p$, the distribution $\mathcal{D}$ is equivalent to a distribution spanned by a family of vector fields that has the following form:

$$
\left(\frac{\partial}{\partial x_{m+k_{0}+k-2}}, \ldots, \frac{\partial}{\partial x_{m+k_{0}+1}}, \xi_{1}^{m+k_{0}}, \xi_{2}^{m+k_{0}}\right)
$$

where the pair of vector fields $\xi^{m+k_{0}}=\left(\xi_{1}^{m+k_{0}}, \xi_{2}^{m+k_{0}}\right)$ is a prolongation of order $k_{0}$ of a pair of vector fields $\xi^{m}=\left(\xi_{1}^{m}, \xi_{2}^{m}\right)$ of the form (49). Moreover, we have $\xi^{m+k_{0}}=\sigma_{k_{0}} \circ \cdots \circ \sigma_{1}\left(\xi^{m}\right)$, where $\sigma_{1}=S$ and each $\sigma_{j}$, for $2 \leq j \leq k_{0}$, equals $R_{c_{j}}$, for some real constants $c_{i}$.

The proof of Lemma D.2 follows the same line as that of Proposition 4.4. Though instead of considering a Kumpera-Ruiz normal form we consider now a family of vector fields of the form (51), the idea is the same. Firstly, we compute the distributions $\mathcal{D}^{(i)}$ and $\mathcal{C}_{i}$, and the submanifolds $S_{0}^{(i)}$. Secondly, we observe that if $\sigma_{1}=R_{c}$, for some real constant $c$, then $p \notin S_{0}^{\left(k_{0}-1\right)}$; since $p \in S_{0}^{\left(k_{0}-1\right)}$ we must have $\sigma_{1}=S$. Thirdly, we observe that if $\sigma_{j}=S$ for some $2 \leq j \leq k_{0}$ then $p \in S_{0}^{\left(k_{0}-j\right)}$; since $k_{0}$ is by definition the smallest integer such that $p \in S_{0}^{\left(k_{0}-1\right)}$ we must have $\sigma_{j}=R_{c_{j}}$, for $2 \leq j \leq k_{0}$.

For Goursat structures, using the singularity type leads to the following stronger result, which states that if the singularity type is of the form $w a_{1} a_{2} \cdots a_{k_{0}}$ then the constants that appear in all regular prolongations in the above lemma equal zero.

Lemma D.3. Let $\mathcal{D}$ be a Goursat structure on a manifold $M$ of dimension $n \geq 5$ and let $p$ be a point in $M$. If the singularity type of $\mathcal{D}$ at $p$ is of the form $w a_{1} a_{2} \cdots a_{k_{0}}$, for some $1 \leq k_{0} \leq n-4$, where $w$ is an arbitrary word of $J_{n-k_{0}-3}$, then $\mathcal{D}$ is locally equivalent to a distribution spanned by a pair of vector fields that has the following form:

$$
\begin{aligned}
\xi_{1}= & \frac{\partial}{\partial x_{1}} \\
\xi_{2}= & x_{1} \frac{\partial}{\partial x_{2}}+\cdots+x_{k_{0}} \frac{\partial}{\partial x_{k_{0}+1}}+\frac{\partial}{\partial x_{k_{0}+2}} \\
& +x_{k_{0}}\left(x_{k_{0}+2} \frac{\partial}{\partial x_{k_{0}+3}}+x_{k_{0}+3} \frac{\partial}{\partial x_{k_{0}+4}}+\sum_{i=k_{0}+5}^{n} \varphi_{i}(x) \frac{\partial}{\partial x_{i}}\right)
\end{aligned}
$$

where the coordinates $x_{1}, \ldots, x_{n}$ are centered at $p$.

The proof of the last lemma follows also the same line as the proof of Proposition 4.4. Again, we leave details to the reader. The main interest of the last lemma is that it gives directly the proof of Lemma 6.6.

Proof of Lemma 6.6. It is straightforward to check that, in the coordinates of Lemma D.3, the canonical submanifold $S_{k_{0}-1}^{\left(k_{0}-1\right)}$ is given by

$$
S_{k_{0}-1}^{\left(k_{0}-1\right)}=\left\{x_{1}=0, \ldots, x_{k_{0}}=0\right\}
$$

and that, moreover, we have $\mathcal{C}_{0}=\left(\xi_{1}\right)$ on $M$ and $\mathcal{A}_{k_{0}-1}^{(0)}(p)=\left(\xi_{2}\right)(p)$ for each point $p$ on $S_{k_{0}-1}^{\left(k_{0}-1\right)}$. In order to obtain the required normal form, we only have to change two coordinates. For $1 \leq i \leq k_{0}+2$ and $k_{0}+5 \leq i \leq n$, take $y_{i}=x_{i}$. Moreover, take $y_{k_{0}+4}=x_{k_{0}+3}$ and $y_{k_{0}+3}=x_{k_{0}+4}-x_{k_{0}+3} x_{k_{0}+1}+\frac{1}{2} x_{k_{0}+2} x_{k_{0}+1}^{2}$. 


\section{Appendix E. Figures of LOW-DimensionAl TRAILER SyStemS}

\section{E.1. The unicycle and the car}

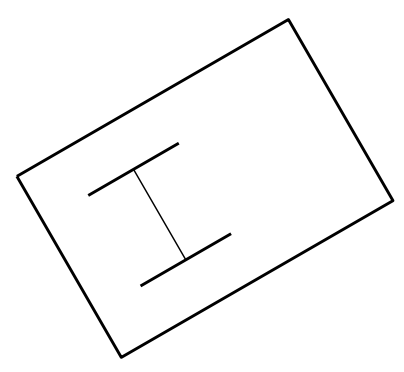

$$
\begin{aligned}
& \dot{x}_{1}=u_{1} \\
& \dot{x}_{2}=x_{1} u_{2} \\
& \dot{x}_{3}=u_{2}
\end{aligned}
$$

Figure 1. The unicycle and its normal form. Growth vector: $(2,3)$. Singularity type: $\epsilon$.

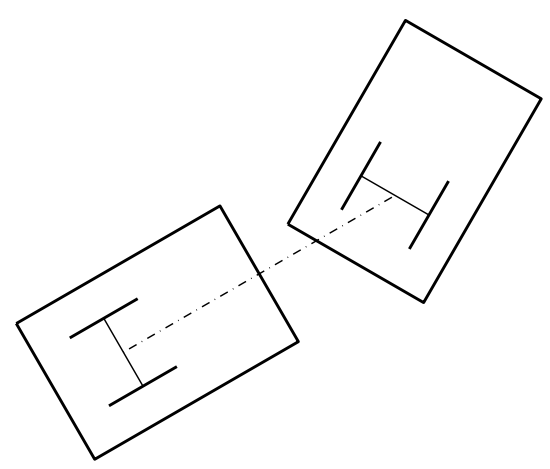

$$
\begin{aligned}
& \dot{x}_{1}=u_{1} \\
& \dot{x}_{2}=x_{1} u_{2} \\
& \dot{x}_{3}=x_{2} u_{2} \\
& \dot{x}_{4}=u_{2}
\end{aligned}
$$

Figure 2. The car and its normal form. Growth vector: $(2,3,4)$. Singularity type: $a_{0}$. 


\section{E.2. The two-trailer system}

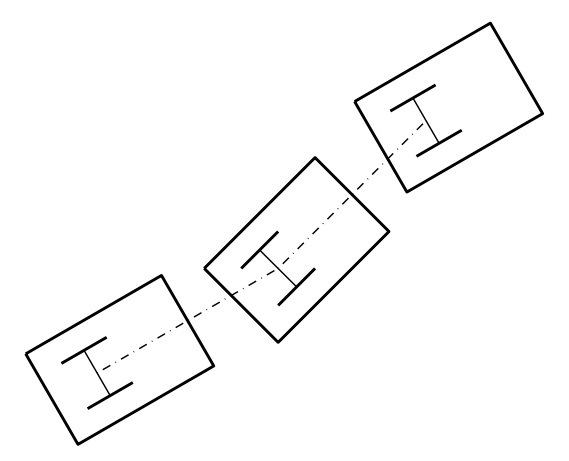

$$
\begin{aligned}
& \dot{x}_{1}=u_{1} \\
& \dot{x}_{2}=x_{1} u_{2} \\
& \dot{x}_{3}=x_{2} u_{2} \\
& \dot{x}_{4}=x_{3} u_{2} \\
& \dot{x}_{5}=u_{2}
\end{aligned}
$$

Figure 3. A two-trailer and its normal form. Growth vector: $(2,3,4,5)$. Singularity type: $a_{0} a_{0}$.

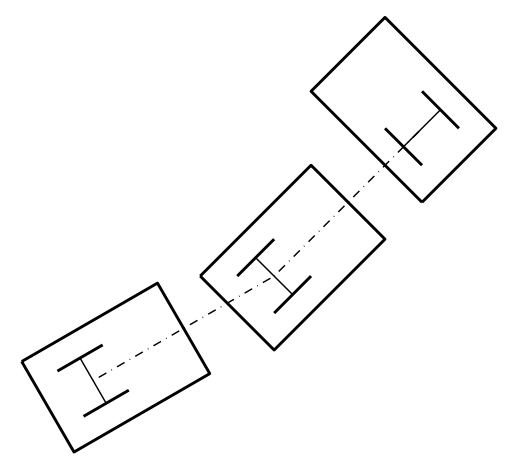

$$
\begin{aligned}
& \dot{x}_{1}=u_{1} \\
& \dot{x}_{2}=u_{2} \\
& \dot{x}_{3}=x_{1} x_{2} u_{2} \\
& \dot{x}_{4}=x_{1} x_{3} u_{2} \\
& \dot{x}_{5}=x_{1} u_{2}
\end{aligned}
$$

Figure 4. A two-trailer and its normal form. Growth vector: $(2,3,4,4,5)$. Singularity type: $a_{0} a_{1}$. 


\section{E.3. The three-trailer system}

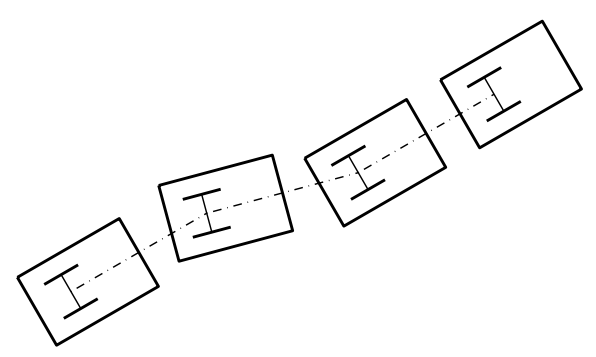

$$
\begin{aligned}
& \dot{x}_{1}=u_{1} \\
& \dot{x}_{2}=x_{1} u_{2} \\
& \dot{x}_{3}=x_{2} u_{2} \\
& \dot{x}_{4}=x_{3} u_{2} \\
& \dot{x}_{5}=x_{4} u_{2} \\
& \dot{x}_{6}=u_{2}
\end{aligned}
$$

Figure 5. A two-trailer and its normal form. Growth vector: $(2,3,4,5,6)$. Singularity type: $a_{0} a_{0} a_{0}$.

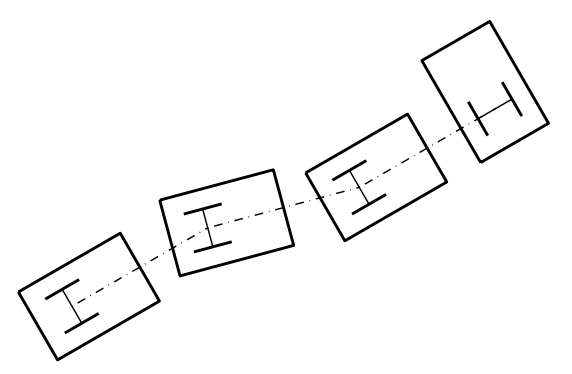

$$
\begin{aligned}
& \dot{x}_{1}=u_{1} \\
& \dot{x}_{2}=u_{2} \\
& \dot{x}_{3}=x_{1} x_{2} u_{2} \\
& \dot{x}_{4}=x_{1} x_{3} u_{2} \\
& \dot{x}_{5}=x_{1} x_{4} u_{2} \\
& \dot{x}_{6}=x_{1} u_{2}
\end{aligned}
$$

Figure 6. A two-trailer and its normal form. Growth vector: $(2,3,4,4,5,5,6)$. Singularity type: $a_{0} a_{0} a_{1}$. 


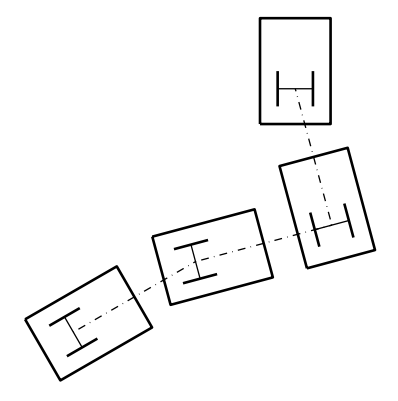

$$
\begin{aligned}
\dot{x}_{1} & =u_{1} \\
\dot{x}_{2} & =\left(x_{1}+1\right) u_{2} \\
\dot{x}_{3} & =u_{2} \\
\dot{x}_{4} & =x_{2} x_{3} u_{2} \\
\dot{x}_{5} & =x_{2} x_{4} u_{2} \\
\dot{x}_{6} & =x_{2} u_{2}
\end{aligned}
$$

FiguRE 7. A two-trailer and its normal form. Growth vector: $(2,3,4,5,5,6)$. Singularity type: $a_{0} a_{1} a_{0}$.

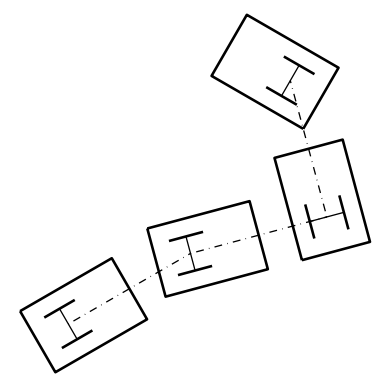

$$
\begin{aligned}
& \dot{x}_{1}=u_{1} \\
& \dot{x}_{2}=x_{1} u_{2} \\
& \dot{x}_{3}=u_{2} \\
& \dot{x}_{4}=x_{2} x_{3} u_{2} \\
& \dot{x}_{5}=x_{2} x_{4} u_{2} \\
& \dot{x}_{6}=x_{2} u_{2}
\end{aligned}
$$

Figure 8. A two-trailer and its normal form. Growth vector: $(2,3,4,5,5,5,6)$. Singularity type: $a_{0} a_{1} a_{2}$.

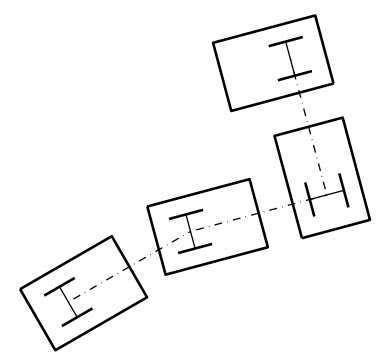

$$
\begin{aligned}
& \dot{x}_{1}=u_{1} \\
& \dot{x}_{2}=u_{2} \\
& \dot{x}_{3}=x_{1} u_{2} \\
& \dot{x}_{4}=x_{1} x_{2} x_{3} u_{2} \\
& \dot{x}_{5}=x_{1} x_{2} x_{4} u_{2} \\
& \dot{x}_{6}=x_{1} x_{2} u_{2}
\end{aligned}
$$

FiguRE 9. A two-trailer and its normal form. Growth vector: $(2,3,4,4,5,5,5,6)$. Singularity type: $a_{0} a_{1} a_{1}$. 


\section{REFERENCES}

[1] A. Agrachev and A. Sarychev, On abnormal extremals for Lagrange variational problems. J. Math. Systems Estim. Control 8 (1998) 87-118.

[2] A. Bäcklund, Über Flachentransformationen. Math. Ann. 9 (1876) 297-320.

[3] B. Bonnard and I. Kupka, Théorie des singularités de l'application entrée/sortie et optimalité des trajectoires singulières dans le problème du temps minimal. Forum Math. (5) (1993) 111-159.

[4] R. Brockett, Control theory and singular Riemannian geometry, edited by P. Hilton and G. Young, New Directions in Applied Mathematics. Springer-Verlag, New York (1981) 11-27.

[5] R. Brockett, Asymptotic stability and feedback stabilization, edited by R. Brockett, R. Millman and H. Sussmann, Differential Geometric Control Theory. Birkhäuser, Boston (1983) 181-191.

[6] R. Bryant, S.-S. Chern, R. Gardner, H. Goldschmidt and P. Griffiths, Exterior Differential Systems. Mathematical Sciences Research Institute Publications. Springer-Verlag, New York (1991).

[7] R. Bryant and L. Hsu, Rigidity of integral curves of rank 2 distributions. Invent. Math. (114) (1993) 435-461.

[8] M. Cañadas-Pinedo and C. Ruiz, Pfaffian systems with derived length one. The class of flag systems. Preprint, University of Granada.

[9] E. Cartan, Sur l'intégration de certains systèmes de Pfaff de caractère deux. Bull. Soc. Math. France 29 (1901) 233 -302. Euvres complètes, Part. II, Vol. 1, Gauthiers-Villars, Paris.

[10] E. Cartan, Les systèmes de Pfaff à cinq variables et les équations aux dérivées partielles du second ordre. Ann. École Norm. Sup. 27 (1910) 108-192. Cuvres complètes, Part. II, Vol. 2, Gauthiers-Villars, Paris.

[11] E. Cartan, Sur l'équivalence absolue de certains systèmes d'équations différentielles et sur certaines familles de courbes. Bull. Soc. Math. France 42 (1914) 12-48. Euvres complètes, Part. II, Vol. 2, Gauthiers-Villars, Paris.

[12] M. Cheaito and P. Mormul, Rank-2 distributions satisfying the Goursat condition: All their local models in dimension 7 and 8. ESAIM: COCV 4 (1999) 137-158.

[13] M. Cheaito, P. Mormul, W. Pasillas-Lépine and W. Respondek, On local classification of Goursat structures. C. R. Acad. Sci. Paris Sér. I Math. 327 (1998) 503-508.

[14] J.-M. Coron, Global asymptotic stabilization for controllable systems without drift. Math. Control Signals Systems 5 (1991) $295-312$.

[15] G. Darboux, Sur le problème de Pfaff. Bull. Sci. Math. 2 (1882) 14-36, 49-68.

[16] F. Engel, Zur Invariantentheorie der Systeme Pfaff'scher Gleichungen. Ber. Verhandlungen der Koniglich Sachsischen Gesellshaft der Wissenshaften Mathematisch-Physikalische Klasse, Leipzig 41 (1889, 1890) 157-176, 192-207.

[17] M. Fliess, J. Lévine, P. Martin and P. Rouchon, Flatness and defect of nonlinear systems: Introductory theory and examples. Int. J. Control 61 (1995) 1327-1361.

[18] G. Frobenius, Über das Pfaff'sche problem. J. Reine Angew. Math. 82 (1877) 230-315.

[19] M. Gaspar, Sobre la clasificacion de sistemas de Pfaff en bandera, in Proc. of the Spanish-Portuguese Conference on Mathematics. Murcia, Spain (1985) 67-74.

[20] A. Giaro, A. Kumpera and C. Ruiz, Sur la lecture correcte d'un resultat d'Élie Cartan. C. R. Acad. Sci. Paris Sér. I Math. 287 (1978) 241-244.

[21] E. Goursat, Sur le problème de Monge. Bull. Soc. Math. France (33) (1905) 201-210.

[22] E. Goursat, Leçons sur le problème de Pfaff. Hermann, Paris (1923).

[23] D. Hilbert, Über den Begriff der Klasse von Differentialgleichungen. Math. Ann. 73 (1912) 95-108.

[24] B. Jacquard, Le problème de la voiture à deux, trois et quatre remorques. Preprint, DMI-ENS Paris (1993).

[25] B. Jakubczyk, Invariants of dynamic feedback and free systems, in Proc. of the European Control Conference. Groningen, The Netherlands (1993) 1510-1513.

[26] B. Jakubczyk, Characteristic varieties of distributions and abnormal curves. Preprint (1999).

[27] B. Jakubczyk and F. Przytycki, Singularities of $k$-tuples of vector fields. Diss. Math. (213) (1984) 1-64.

[28] B. Jakubczyk and M. Zhitomirskiŭ, Odd-dimensional Pfaffian equations: Reduction to the hypersurface of singular points. C. R. Acad. Sci. Paris Sér. I Math. 325 (1997) 423-428.

[29] F. Jean, The car with $n$ trailers: Characterization of the singular configurations. ESAIM: COCV 1 (1996) 241-266.

[30] Z.-P. Jiang and H. Nijmeijer, A recursive technique for tracking control of nonholonomic systems in chained form. IEEE Trans. Automat. Control 44 (1999) 265-279.

[31] M. Kazarian, R. Montgomery and B. Shapiro, Characteristic classes for the degenerations of two-plane fields in four dimensions. Pacific J. Math. 179 (1997) 355-370.

[32] A. Kumpera and C. Ruiz, Sur l'équivalence locale des systèmes de Pfaff en drapeau, edited by F. Gherardelli, Monge-Ampère equations and related topics. Instituto Nazionale di Alta Matematica Francesco Severi, Rome (1982) 201-247.

[33] G. Lafferriere and H. Sussmann, A differential geometric approach to motion planning, Nonholonomic motion planning, edited by Z. Li and J. F. Canny, International Series in Engineering and Computer Sciences. Kluwer, Dordrecht (1992) $235-270$.

[34] J.-P. Laumond, Controllability of a multibody mobile robot. IEEE Trans. Robotics and Automation 9 (1991) $755-763$. 
[35] J.-P. Laumond, Singularities and topological aspects in nonholonomic motion planning, edited by Z. Li and J.F. Canny, Nonholonomic motion planning, International Series in Engineering and Computer Sciences. Kluwer, Dordrecht (1992) 755763.

[36] J.-P. Laumond, Robot Motion Planning and Control. Springer-Verlag, Berlin, Lecture Notes on Control and Information Sciences (1997).

[37] J.-P. Laumond, P. Jacobs, M. Taïx and R. Murray, A motion planner for nonholonomic mobile robots. IEEE Trans. Robotics and Automation 10 (1994) 577-593.

[38] Z. Li and J.-F. Canny, Nonholonomic Motion Planning, International Series in Engineering and Computer Sciences. Kluwer, Dordrecht (1992).

[39] P. Libermann, Sur le problème d'équivalence des systèmes de Pfaff non complètement intégrables. Publ. Paris VII 3 (1977) $73-110$.

[40] S. Lie and G. Scheffers, Geometrie of Berührungstransformationen. B. G. Teubners, Leipzig (1896).

[41] W. Liu, An approximation algorithm for non-holonomic systems. SIAM J. Control Optim. 35 (1997) $1328-1365$.

[42] F. Luca and J.-J. Risler, The maximum degree of nonholonomy for the car with $n$ trailers, in Proc. of the IFAC Symposium on Robot Control. Capri, Italy (1994) 165-170.

[43] P. Martin and P. Rouchon, Feedback linearization and driftless systems. Math. Control Signals Systems 7 (1994) $235-254$.

[44] R. M'Closkey and R. Murray, Exponential stabilization of driftless nonlinear control systems using homogeneous feedback. IEEE Trans. Automat. Control 42 (1997) 614-628.

[45] R. Montgomery, A survey of singular curves in sub-Riemannian geometry. J. Dynam. Control Systems (1995) 49-90.

[46] R. Montgomery and M. Zhitomirskiǔ, Geometric approach to Goursat flags. Preprint, University of California Santa Cruz (1999).

[47] P. Morin and C. Samson, Exponential stabilization of nonlinear driftless systems with robustness to unmodeled dynamics. ESAIM: COCV 4 (1999) 1-35.

[48] P. Mormul, Contact hamiltonians distinguishing locally certain Goursat systems. Preprint, Warsaw (1998).

[49] P. Mormul, Local models of 2-distributions in 5 dimensions everywhere fulfilling the Goursat condition. Research report, Rouen (1994).

[50] P. Mormul, Rank-2 distributions satisfying the Goursat condition: All their local models in dimension 9. Preprint, Institute of Mathematics, Polish Academy of Sciences (1997).

[51] P. Mormul, Goursat distributions with one singular hypersurface - constants important in their Kumpera-Ruiz pseudo-normal forms. Preprint, Université de Bourgogne (1999).

[52] P. Mormul, Goursat flags: Classification of codimension-one singularities. Preprint, Warsaw University (1999).

[53] R. Murray, Nilpotent bases for a class of nonintegrable distributions with applications to trajectory generation for nonholonomic systems. Math. Control Signals Systems 7 (1994) 58-75.

[54] R. Murray and S. Sastry, Nonholonomic motion planning: Steering using sinusoids. IEEE Trans. Automat. Control 38 (1993) $700-716$.

[55] P. Olver, Equivalence, Invariants, and Symmetry. Cambridge University Press (1995).

[56] W. Pasillas-Lépine and W. Respondek, Applications of the geometry of Goursat structures to nonholonomic control systems, in Proc. of the IFAC Nonlinear Control Systems Design Symposium. Enschede, The Netherlands (1998) 789-794.

[57] W. Pasillas-Lépine and W. Respondek, Conversion of the n-trailer into Kumpera-Ruiz normal form and motion planning through the singular locus, in Proc. of the IEEE Conference on Decision and Control. Phoenix, Arizona (1999) $2914-2919$.

[58] J.-B. Pomet, Explicit design of time-varying stabilizing control laws for a class of controllable systems without drift. Systems Control Lett. 18 (1992) 147-158.

[59] L. Pontryagin, V. Boltyanskiı̌, R. Gamkrelidze and E. Mischenko, The Mathematical Theory of Optimal Processes. Wiley, New York (1962).

[60] P. Rouchon, M. Fliess, J. Lévine and P. Martin, Flatness and motion planning: The car with $n$ trailers, in Proc. of the European Control Conference. Groningen (1993) 1518-1522.

[61] C. Samson, Control of chained systems: Application to path following and time-varying point-stabilization of mobile robots. IEEE Trans. Automat. Control 40 (1995) 64-77.

[62] O. Sørdalen, Conversion of the kinematics of a car with $n$ trailers into a chained form, in Proc. of the IEEE Conference on Robotics and Automation. Atlanta, Georgia (1993) 382-387.

[63] O. Sørdalen, On the global degree of nonholonomy of a car with $n$ trailers, in Proc. of the IFAC Symposium on Robot Control. Capri, Italy (1994) 343-348.

[64] O. Sørdalen and O. Egeland, Exponential stabilization of nonholonomic chained systems. IEEE Trans. Automat. Control 40 (1995) 35-49.

[65] O. Sørdalen, Y. Nakamura and W. Chung, Design and control of a nonholonomic manipulator, in École d'été d'automatique de l'ENSIEG. Grenoble, France (1996).

[66] O. Sørdalen and K. Wichlund, Exponential stabilization of a car with $n$ trailers, in Proc. of the IEEE Conference on Decision and Control. San Antonio, Texas (1993) 978-983. 
[67] H. Sussmann and W. Liu, Shortest paths for sub-Riemannian metrics of rank-2 distributions. Mem. Amer. Math. Soc. 192 (1995).

[68] A. Teel, R. Murray and G. Walsh, Nonholonomic control systems: From steering to stabilization with sinusoids. Int. J. Control 62 (1995) 849-870.

[69] D. Tilbury, R. Murray and S. Sastry, Trajectory generation for the $n$-trailer problem using Goursat normal form. IEEE Trans. Automat. Control 40 (1995) 802-819.

[70] A. Vershik and V. Gershkovich, Nonholonomic dynamical systems, geometry of distributions and variational problems, edited by V. Arnol'd and S. Novikov, Dynamical systems VII, Encyclopaedia of Mathematical Sciences. Springer-Verlag, New-York (1991).

[71] G. Walsh, D. Tilbury, S. Sastry, R. Murray and J.-P. Laumond, Stabilization of trajectories for systems with nonholonomic constraints. IEEE Trans. Automat. Control 39 (1994) 216-222.

[72] E. von Weber, Zur Invariantentheorie der Systeme Pfaff'scher Gleichungen. Ber. Verhandlungen der Koniglich Sachsischen Gesellshaft der Wissenshaften Mathematisch-Physikalische Klasse, Leipzig 50 (1898) 207-229.

[73] I. Zelenko and M. Zhitomirskiĭ, Rigid paths of generic 2-distributions on 3-manifolds. Duke Math. J. 79 (1995) $281-307$.

[74] P. Zervos, Le problème de Monge. Mémorial des Sciences Mathématiques. Gauthier-Villars, Paris (1932).

[75] M. Zhitomirskiü, Normal forms of germs of 2-dimensional distributions on $R^{4}$. Funct. Analys. Appl. 24 (1990) $150-152$.

[76] M. Zhitomirski1̌, Normal forms of germs of distributions with a fixed segment of growth vector. Leningrad Math. J. (2) (1991) 1043-1065 (English translation).

[77] M. Zhitomirskiŭ, Rigid and abnormal line subdistributions of 2-distributions. J. Dynam. Control Systems (1) (1995) $253-294$.

[78] M. Zhitomirskiǔ, Singularities and normal forms of smooth distributions, edited by B. Jakubczyk, W. Respondek and T. Rzezuchowski, Geometry in Nonlinear Control and Differential Inclusions. Banach Center Publications, Warszawa (1995) 395-409. 